\title{
Explicit examples of DIM constraints for network matrix models
}

\author{
Hidetoshi Awata, ${ }^{a}$ Hiroaki Kanno, ${ }^{a, b}$ Takuya Matsumoto, ${ }^{a}$ Andrei Mironov, $, c, d, e, f$ \\ Alexei Morozov, ${ }^{d, e, f}$ Andrey Morozov, ${ }^{e, f, g}$ Yusuke Ohkubo $^{a}$ and Yegor Zenkevich ${ }^{d, h, f}$ \\ ${ }^{a}$ Graduate School of Mathematics, Nagoya University, \\ Nagoya, 464-8602, Japan \\ ${ }^{b}$ KMI, Nagoya University, \\ Nagoya, 464-8602, Japan \\ ${ }^{c}$ Theory Department, Lebedev Physics Institute, \\ Leninsky pr., 53, Moscow 119991, Russia \\ ${ }^{d}$ ITEP, \\ Bol.Cheremushkinskaya, 25, Moscow 117218, Russia \\ e Institute for Information Transmission Problems, \\ Bol.Karetny, 19 (1), Moscow 127994, Russia \\ ${ }^{f}$ National Research Nuclear University MEPhI, \\ Kashirskoe sh., 31, Moscow 115409, Russia \\ ${ }^{g}$ Laboratory of Quantum Topology, Chelyabinsk State University, \\ Bratiev Kashirinyh, 129, Chelyabinsk 454001, Russia \\ ${ }^{h}$ Institute for Nuclear Research, \\ 60-letiya Oktyabrya pr., 7a, Moscow 117312, Russia \\ E-mail: awata@math.nagoya-u.ac.jp, kanno@math.nagoya-u.ac.jp, \\ takuya.matsumoto@math.nagoya-u.ac.jp, mironov@lpi.ru, \\ morozov@itep.ru, andrey.morozov@itep.ru, m12010t@math.nagoya-u.ac.jp, \\ yegor.zenkevich@gmail.com
}

ABSTRACT: Dotsenko-Fateev and Chern-Simons matrix models, which describe Nekrasov functions for SYM theories in different dimensions, are all incorporated into network matrix models with the hidden Ding-Iohara-Miki (DIM) symmetry. This lifting is especially simple for what we call balanced networks. Then, the Ward identities (known under the names of Virasoro $/ \mathcal{W}$-constraints or loop equations or regularity condition for $q q$-characters) are also promoted to the DIM level, where they all become corollaries of a single identity.

KeYwords: Conformal and W Symmetry, Supersymmetric gauge theory, Topological Field Theories, Topological Strings

ARXIV EPRINT: 1604.08366 


\section{Contents}

1 Introduction $\quad 2$

2 Basic example: theme with variations $\quad 8$

2.0 The main theme 8

2.1 Variation I: matrix elements in the free-field theory 10

$\begin{array}{ll}2.2 \text { Variation II: generating functions } & 11\end{array}$

2.3 Variation III: DF model 12

2.4 Variation IV: multi-field case 13

2.5 Variation V: Chern-Simons (CS) model 15

\begin{tabular}{ll}
2.6 & Variation VI: correlators with vertex operators \\
\hline
\end{tabular}

$\begin{array}{ll}2.7 & \text { Variation VII: Nekrasov functions } \\ & 17\end{array}$

$\begin{array}{lll}2.8 & \text { Variation VIII: network model level. Network as a Feynman diagram } & 18\end{array}$

$\begin{array}{ll}2.9 \text { Variation IX: balanced network model } & 19\end{array}$

2.10 Variation X: $q$-deformation 21

2.11 Variation XI: $q, t, \ldots$-deformations 22

2.12 Variation XII: $\beta$-deformation to non-unit Virasoro central charge 23

3 DIM calculus for balanced network model $\quad 25$

$\begin{array}{lll}3.1 & \text { DIM algebra } & 25\end{array}$

$\begin{array}{lll}3.2 & \text { Bosonization in the case of special slopes } & 27\end{array}$

$\begin{array}{lll}3.3 & \text { Relation to topological vertex } & 28\end{array}$

$\begin{array}{ll}3.4 & \text { Building screening charges and vertex operators }\end{array}$

3.5 Network partition function 34

3.6 Examples of conformal blocks $\quad 35$

$\begin{array}{lll}3.7 & \text { Compactified network and the affine screening operator } & 39\end{array}$

4 The action of Virasoro and $\operatorname{DIM}\left(\mathfrak{g l}_{1}\right) \quad 40$

5 Vertical action of DIM $\quad 43$

6 Conclusion $\quad 44$

A Properties of the DIM algebras and their limits 46

A.1 Constructing $\operatorname{DIM}\left(\mathfrak{g l}_{1}\right)$ from $W_{1+\infty}$ algebra 46

A.2 Elliptic $\operatorname{DIM}\left(\mathfrak{g l}_{1}\right)$ algebra $\quad 49$

A.3 Rank > 1: $\operatorname{DIM}\left(\mathfrak{g l}_{n}\right)=$ quantum toroidal algebra of type $\mathfrak{g l}_{n} \quad 50$

A.4 Affine Yangian of $\mathfrak{g l}_{1}[139] \quad 51$ 


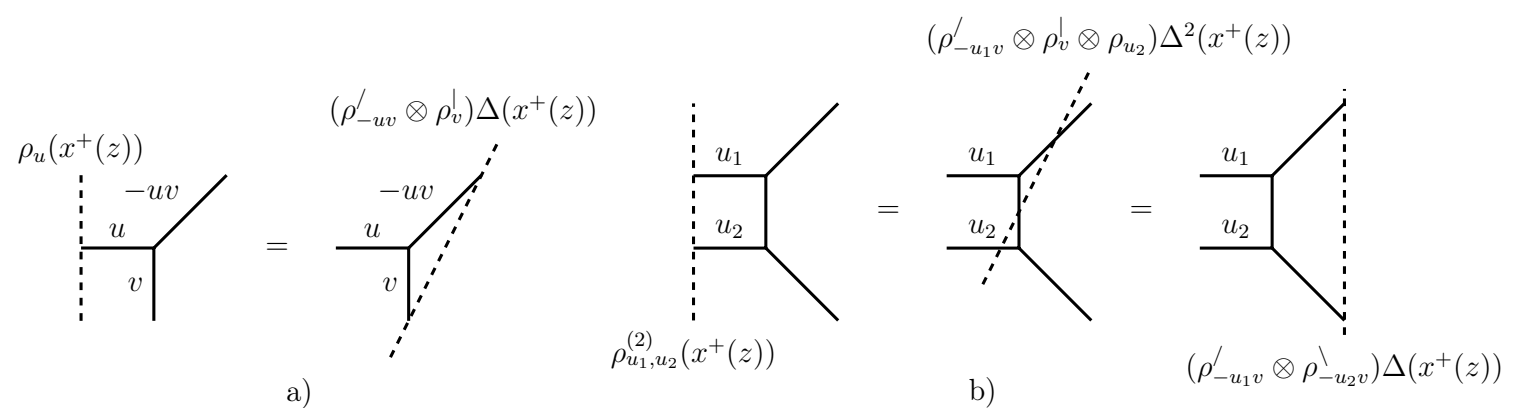

Figure 1. Topological vertex as the intertwiner of DIM representations. a) The action of the generator $x^{+}(z)$ on the level one Fock representation $\rho_{u}$ sitting on the horizontal leg of the topological vertex (denoted by the dashed line) is the same as its action on the product of two representations — the "vertical" $\rho_{v}^{l}$ and "diagonal" $\rho_{-u v}^{\prime}$. b) Appropriate contraction of two intertwiners is also an intertwiner. This gives the vertex operator of the corresponding conformal field theory with deformed Virasoro symmetry, corresponding to a single vertical brane in figure 2.

\section{Introduction}

Nekrasov functions, describing instanton corrections in supersymmetric Yang-Mills theories [1]-[11], and AGT related conformal blocks [12-16] possess rich symmetries that can be separated into large and infinitesimal. The former describe dualities between different models, while the latter define equations on the partition functions in each particular case. They are also known as "Virasoro constraints" [17, 18] for associated conformal or Dotsenko-Fateev (DF) matrix models [19-30], which are further promoted to network matrix models [31-34], looking like convolutions of refined topological vertices [35-37] and possessing direct topological string interpretation.

As conjectured in a number of papers throughout recent years [38]-[49] and recently summarized in [50], in full generality the symmetry underlying the AGT correspondence [51-53], is the Ding-Iohara-Miki algebra (DIM) [54]-[69], in particular, the infinitesimal Ward identities are controlled by DIM from which the (deformed) Virasoro and $\mathcal{W}_{K}$ emerge as subalgebras in particular representations. In other words, the full symmetry of the Seiberg-Witten theory seems to be the Pagoda triple-affine elliptic DIM algebra (not yet fully studied and even defined), and particular models (brane patterns or Calabi-Yau toric varieties labeled by integrable systems a $l a[3,4])$ are associated with its particular representations. The ordinary DF matrix models arise when one specifies "vertical" and "horizontal" directions, then convolutions of topological vertices can be split into vertex operators and screening charges, and the DIM algebra constraints can be attributed in the usual way [70-79] to commutativity of screening charges with the action of the algebra in the given representation. Dualities are associated with the change of the vertical/horizontal splitting, or, more general, with the choice of the section, where the algebra acts [80-82].

All this is illustrated in pictures 1 and 2, which we borrowed from [50], and our purpose in this paper is to provide very explicit examples of how these pictures are converted into formulas. A great deal of these formulas already appeared in the literature. Putting them together, we hope to illustrate their general origin and better formulate the remaining open problems. 


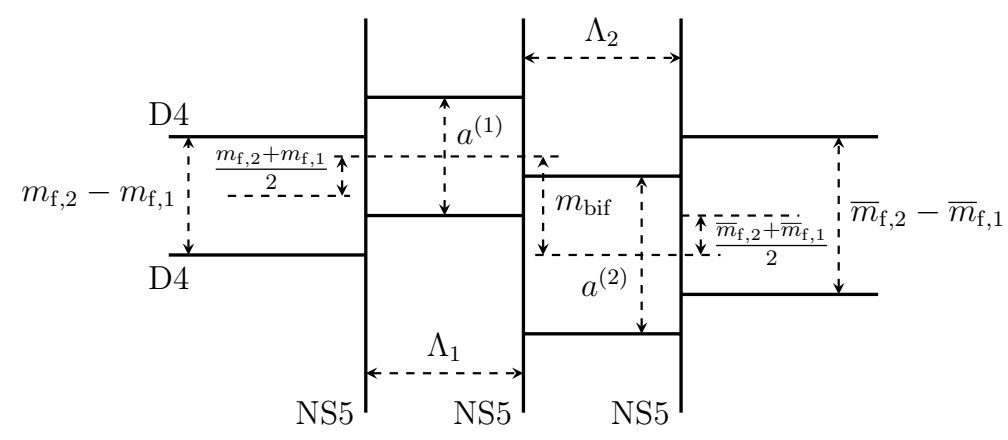

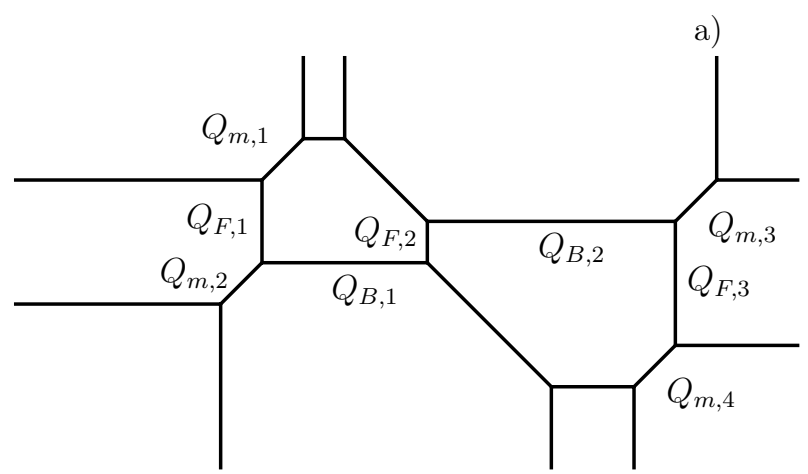

b)

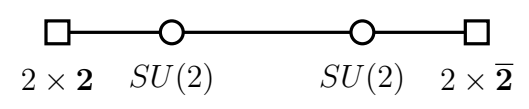

c)

Figure 2. a) Type IIA brane diagram consisting of two horizontal and three vertical intersecting lines representing NS5 and D4 branes. The low energy theory in this background is $4 \mathrm{~d} \mathcal{N}=2$ gauge theory with $\mathrm{SU}(2)^{2}$ gauge group. $\Lambda_{i}$ are exponentiated complexified gauge couplings, $a^{(a)}$ are Coulomb moduli and $m_{a}$ are the hypermultiplet masses. b) The toric diagram of the Calabi-Yau threefold, corresponding to the $5 d$ gauge theory with the same matter content. Edges represent twocycles with complexified Kähler parameters $Q_{i}$, which play the same role as the distances between the branes in a). c) The quiver encoding the matter content of the gauge theory. SU(2) gauge groups live on each node and bifundamental matter on each edge. The squares represent pairs of (anti)fundamental matter hypermultiplets.

The main scheme could be formulated as follows:

- To build a functor

rank- $r$ Lie algebra $G \longrightarrow$ quantized double-center double-loop $\operatorname{DIM}(G)$, perhaps, $q_{123 \ldots}$-dependent and elliptic

- To obtain a non-linear Sugawara construction of stress tensor and other symmetry generators from a comultiplication $\Delta_{D I M}$.

- To clarify the interplay between two "orthogonal" ("horizontal" and "vertical") comultipilcations.

- To apply the functor (1.1) to central-extended loop algebras $G$, starting from $G=$ $\widehat{(g l(1)}$, to obtain triple-affine Pagoda DIM algebras. One of the immediate problems 
is that the known construction of $\operatorname{DIM}(G)$ for non-affine $g l_{N}$ algebras $[54,83-85]$ already involves the affine Dynkin diagrams, thus, for an affine $\widehat{g l_{N}}$ one can need something more sophisticated.

- An additional light on the problem can be shed by comparative analysis of $\operatorname{DIM}\left(g l_{2}\right)$, $\operatorname{DIM}\left(g l_{3}\right), \operatorname{DIM}\left(s o_{5}\right), \operatorname{DIM}\left(g_{2}\right)$ and $\operatorname{DIM}\left(\widehat{g l_{1}}\right)$, first four of them being explicitly constructed, and by studying their various limits including the one to the affine Yangian and further to the standard conformal algebras (coset constructions of conformal field theories, [86]).

Actually, the first three issues are actively studied by various authors (and there has been already achieved a serious progress), and we do not achieve too much in the two last challenging directions in the present paper, which can be considered as an introduction to the problem. What we actually do, is search for a $q, t$-deformed network analogue of the CFT Ward identity [12]

$$
\left\langle\prod_{a} \hat{V}_{\alpha_{a}}\left(z_{a}\right) \cdot \hat{\mathcal{T}}_{+}(z) \cdot \hat{\mathcal{Q}}^{r}\right\rangle=0
$$

where $\langle\ldots\rangle$ denotes the matrix element $\langle v a c|\ldots| v a c\rangle$ between two vacua of operators in the fixed chronological order and in the chiral sector [87, 88]. Here $V_{\alpha}(z)$ is a primary field (vertex operator) in the free field $c=1 \mathrm{CFT}, \mathcal{T}(z)$ is its stress-energy tensor and $\mathcal{Q}$ is the corresponding screening charge [70-72], which is the integral $\mathcal{Q}=\oint_{x} \mathcal{S}(x)$ of the screening current $\mathcal{S}(x)$.

The order of operators in (1.2) means that in the conformal correlator

$$
\left\langle\left\langle\prod_{a} V_{\alpha_{a}}\left(z_{a}\right) \mathcal{T}_{+}(z) \mathcal{Q}^{r}\right\rangle\right\rangle
$$

(where $<<\ldots>>$ denotes the chiral part of the CFT correlator) all $\left|z_{a}\right|>|z|$ and $|z|>\left|x_{i}\right|$, where $x_{i}$ 's lies on the integration contours of the screening currents.

The Ward identity (1.2) can be manifestly written as

$$
\begin{aligned}
& z^{2} \oint_{x_{i}}\left(\frac{\left.\sum_{a, b} \frac{1}{4} \frac{\alpha_{a} \alpha_{b}}{\left(z-z_{a}\right)\left(z-z_{b}\right)}+\sum_{a, i} \frac{\alpha_{a}}{\left(z-z_{a}\right)\left(z-x_{i}\right)}+\sum_{i, j=1}^{r} \frac{1}{\left(z-x_{i}\right)\left(z-x_{j}\right)}\right)}{\quad \times\left\langle\left\langle\prod_{a} V_{\alpha_{a}}\left(z_{a}\right) \prod_{i=1}^{r} \mathcal{S}\left(x_{i}\right)\right\rangle\right\rangle=\operatorname{Pol}(z)}\right.
\end{aligned}
$$

and the notation $\operatorname{Pol}(z)$ means a power series, i.e. any positive powers of $z$ are allowed. The underlined terms just contribute to $\operatorname{Pol}(z)$ (since $\left|z_{a}\right|>|z|$ ) and can be omitted giving finally

$$
z^{2} \oint_{x_{i}}\left(\sum_{a, i} \frac{\alpha_{a}}{\left(z-z_{a}\right)\left(z-x_{i}\right)}+\sum_{i, j=1}^{r} \frac{1}{\left(z-x_{i}\right)\left(z-x_{j}\right)}\right)\left\langle\left\langle\prod_{a} V_{\alpha_{a}}\left(z_{a}\right) \prod_{i=1}^{r} \mathcal{S}\left(x_{i}\right)\right\rangle\right\rangle=\operatorname{Pol}(z)
$$


Though equivalent, (1.2) and (1.5) are in fact very different. The second one is about field theory correlators, it is dictated by operator expansions and is especially simple because a free field formalism is available for conformal theories. The first one is actually about matrix elements, and the difference is that it depends on the ordering of operators, while correlators do not. Another way to say this is that the projected stress tensor $\mathcal{T}_{+}(z)$ does not have a simple operator product expansion (OPE) with other operators, the projection is a non-local operation and actually depends on the position: if $\mathcal{T}_{+}(z)$ was placed to the left of vertex operators $V\left(z_{a}\right)$, the matrix element would no longer vanish. At the same time, in this case the underlined terms in (1.4) also contribute (since $|z|>\left|z_{a}\right|$ ), and they exactly cancel non-zero matrix element leading to the same Ward identity (1.5).

These are trivial remarks for the old-fashioned field theory, where the Ward identities were discovered and treated as sophisticated recurrence relations between Feynman diagrams, but in modern CFT we got used to the formalism based on the operator product expansion and moving the integration contours, which provides a shortcut for the derivations. Unfortunately, in the network models, only the operator approach is currently available, and this is the reason why we need to develop the formalism from this starting point.

Still, some elements of the free field formalism are already worked out in particular representations of DIM, and for a special class of balanced network models, drawn as a set of horizontal lines with vertical segments in between, see figure 3, a), one has a direct counterpart of (1.2). In (extremely) condensed notation it looks like

$$
\left\langle\prod_{a} \Psi_{\lambda_{a}}\left[z_{a}\right] \Psi_{\mu_{a}}^{*}\left[z_{a}^{*}\right] \hat{\mathcal{T}}_{+}(z ; u \mid \xi) \quad \prod_{b}\left(\sum_{\mu} \Psi_{\mu} \Psi_{\mu}^{*}\right)\right\rangle=0
$$

and involves operators like

$$
\prod_{I} \Psi_{\lambda_{I}}\left[z_{I}\right] \prod_{J} \Psi_{\mu_{J}}^{*}\left[z_{J}^{*}\right] \longrightarrow \prod_{I, J} \exp \left(\sum_{n \neq 0} \frac{1}{n}\left(\omega^{|n|}\left[\lambda_{I}, z_{I}\right]_{n} \mathfrak{a}_{n}-\left[\mu_{J}, z_{J}^{*}\right]_{n} \mathfrak{a}_{n}^{*}\right)\right)
$$

where

$$
[\lambda, z]_{n} \equiv \operatorname{sign}(n) \sum_{i}\left(q^{\lambda_{i}-1 / 2} t^{1 / 2-i} z\right)^{n}
$$

are the Miwa variables associated with the Young diagram $\lambda$, and the Drinfeld-Sokolov operator (generalized stress energy tensor $=$ Miura transformation from $\Lambda_{i}(z)$ )

$$
\begin{aligned}
\hat{\mathcal{T}}(z ; u \mid \xi) & =z^{1 / 2 \log _{\omega} \xi}: \prod_{i=1}^{K}\left(\omega^{-2 z \partial_{z}}-u_{i} \Lambda_{i}\left(z \omega^{2(i-1)}\right)\right): \\
z^{-1 / 2 \log _{\omega} \xi} & =\sum_{k=1}^{K} \xi^{K-k} \sum_{i_{1}<\ldots<i_{M}} \prod_{a=1}^{k} u_{i_{a}}: \Lambda_{i_{a}}\left(z \omega^{2(a-1)}\right):
\end{aligned}
$$

defining numerous flows, is a linear combination of all $\mathcal{W}^{(m)}$ with $m \leq K$. Here $\Lambda_{i}(z)$ are also made from the annihilation and creation operators $\hat{\alpha}_{ \pm n}, \omega=\sqrt{q / t}$ and $\hat{\mathcal{T}}(z ; u \mid \xi)$ depends on an additional parameter $\xi$ generating different $\mathcal{W}^{(m)}(z)$ and on spectral parameters of DIM representation $u_{i}$. 


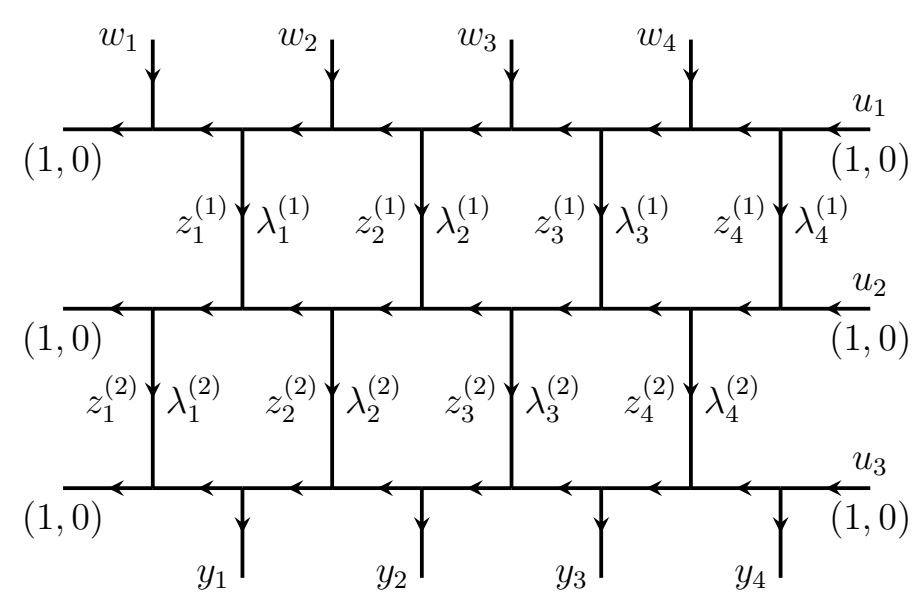

a)

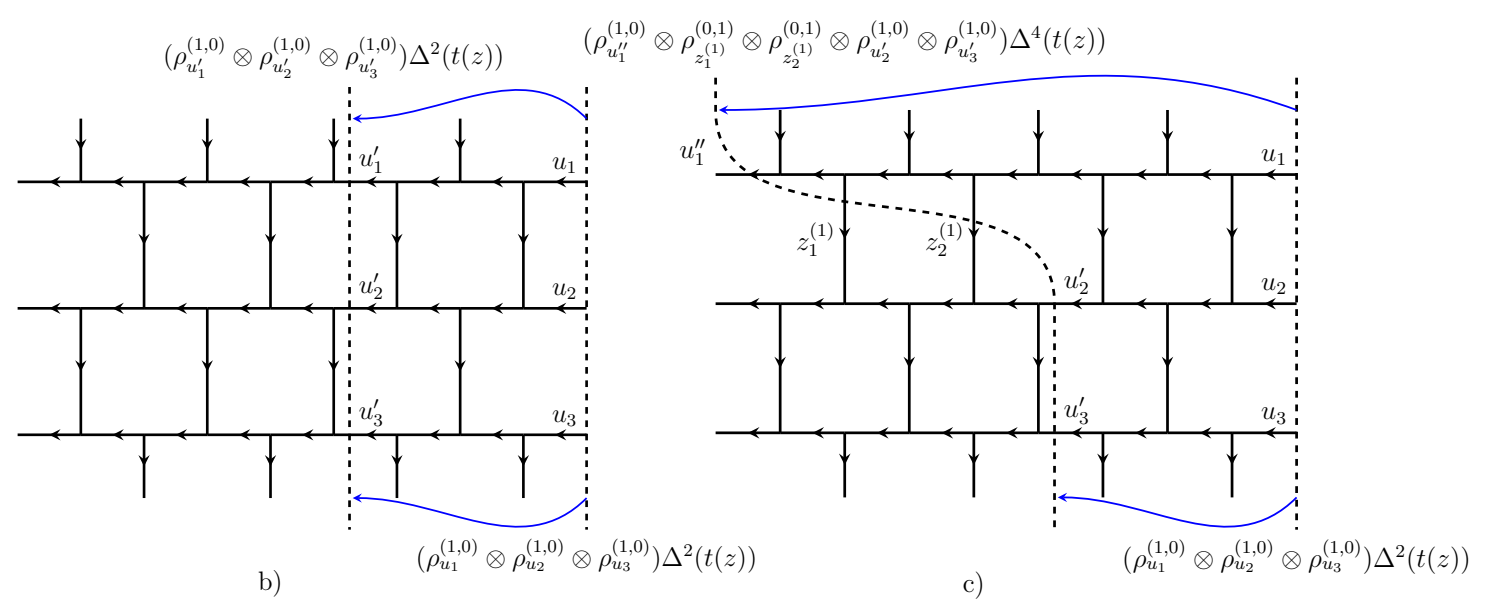

Figure 3. a) An example of balanced network. Notice that the numbers of incoming and outgoing vertical branes are the same in each horizontal section. Because of this, the slopes of the horizontal branes have the same slopes $(1,0)$ to the left and to the right of the diagram. b) The action of a DIM algebra element on the section of the diagram.

A counterpart of (1.2) emerges when the dashed vertical section in figure $3, \mathrm{~b}$ ) is shifted to the left, through external vertical legs, which do not commute with $\hat{\mathcal{T}}(z)$. Moreover, now we can also consider deformations of the section which do not preserve verticality, like the dotted one in figure 3, c), and everything can still be calculated. This should provide a qualitatively new insight into spectral dualities [89-94] associated with global rotations of the network graph.

Non-balanced networks, where the right-most and left-most branes in figure 4 are tilted and the number of operators $\Psi$ differs from that of $\Psi^{*}$, can be considered as certain limits of the balanced ones, but these limits are non-trivial and singular when, say, $q, t \longrightarrow 1$. From the point of view of representation theory these limits should have independent description, making use of more complicated intertwiners. A full-fledged free field description for them comparable to the one in [95-97] for ordinary affine case still needs to be worked out. 
Restriction to the balanced networks is a great technical simplification, but it requires a somewhat lengthy comments on what this means and whether this really restricts the set of handy physical models.

DIM is a quantization of double loop (double affine) algebras, and the existing free field formalism, which we are going to expose and exploit in the present paper, explicitly breaks the symmetry between the two loops. Bosonized/fermionized are only the Chevalley generators, in the case of DIM there are many, still they depend on one of the two loop parameters, while the other loop is associated with their multiple commutators and is described very differently: in terms of Young diagrams parameterizing states in the Fock space. This breaks the symmetry of the DIM algebra: the $\mathrm{SL}(2, \mathbb{Z})$-automorphisms acting on the square lattice of the generators and introduces asymmetry between horizontal and vertical directions in the planar graphs which are used to define the network models, and makes the spectral dualities interchanging these two directions highly non-trivial. In particular, allowed networks look like infinite "horizontal" lines, connected by vertical segments, see figure 4, a), and not vice versa. We call these lines horizontal, though they can have varying slopes, however, they have a non-trivial projection on the horizontal axis, i.e. are strictly non-vertical. In the original brane theory interpretation these horizontal lines depict the $D$-branes, while vertical are the $N S$ branes, from this point of view our description applies only to the conformal models $\left(N_{f}=2 N_{c}\right)$ with definite $N_{c}=M=\#$ of horizontal lines. Quiver models $\otimes \mathrm{SU}\left(N_{i}\right)$ with different $N_{i}$ can seem excluded, but in fact they appear after application of the spectral duality: a $90^{\circ}$ rotation of the graph, see Fig, 4, b). After this rotation, the infinite horizontal lines get associated with the infinite $N S$ branes, while the vertical segments with $D$-branes between them. This pattern looks more relevant from the gauge theory point of view, but we emphasize that our free fields live on the infinite horizontal lines, the three-valent vertices (the DIM algebra intertwiners $\Psi$ and $\Psi^{*}$, also known as topological vertices) act as operators in the Fock spaces horizontally, while the third vertical edge carries a Young-diagram label, not converted into operator language. In result these vertices can look like $\perp$ or $\top$, but not like $\vdash$ or $\dashv$.

All these restrictions can be lifted by switching from Fock to MacMahon modules, which are representations of DIM spanned by $3 \mathrm{~d}$ partitions, but such a description is only combinatorial so far, no generalization to the full-fledged double-loop free field formalism is available yet. This is what makes tedious the consideration of dotted sections in figure $3, \mathrm{c}$ ). We briefly touch this issue at the very end of this text, but detailed presentation is postponed to the future work. Our main purpose here is to describe the powerful free field formalism for the balanced network as a straightforward generalization of that for the ordinary conformal theories, and explain how the DIM algebra becomes the symmetry of generic Nekrasov functions generalizing the Virasoro/W symmetry of the ordinary conformal blocks and Dotsenko-Fateev matrix models.

In the next section 2, we explain how the elementary theory of a harmonic oscillator can be straightforwardly developed and lifted to description of generic networks, i.e. of generic Nekrasov functions. In section 3, in the simplest examples we demonstrate the actual formalism in full detail. It is important that most complications come from sophisticated notation, which are largely no more than a change of variables (normalization 


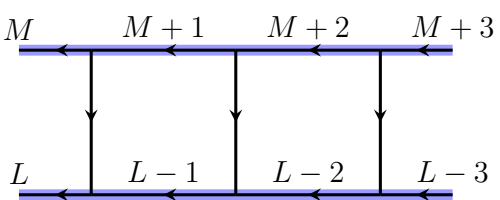

a)

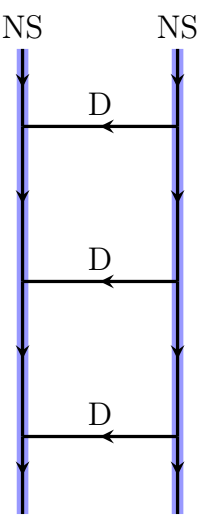

b)

Figure 4. a) An example of non-balanced web with infinite "horizontal" lines shown in blue. Bending of the "horizontal" lines due to tension from the vertical segments is reflected in their slopes marked above them. b) Spectral duality acts by rotating the diagram a). After rotation one can identify the conventional Hanany-Witten (or brane web/geometric engineering) setup with NS5 and D5-branes ().

of creation and annihilation operators). The really big change comes in section 4, when one looks at the symmetry: it is indeed essentially deformed. But this deformation actually simplifies things, reducing all the symmetries to the action of the DIM generators, while the Sugawara construction of Virasoro and W-operators and of their sophisticated $q$-deformations is no more than the simple comultiplication rule. At last, at section 5 we briefly discuss the spectral duality action on symmetry generators. Finally, the appendix contains further details about various DIM algebras and their representations. At present stage of development, different parameters are treated as providing different algebras, but further studies can promote them to parameters of different representations of a single unified algebra (like the triple-affine elliptic Pagoda DIM algebra anticipated in [50]).

Notation. Throughout the text we use the notation

$$
\omega \equiv \sqrt{\frac{q}{t}}
$$

\section{Basic example: theme with variations}

We assume some familiarity with [50] and do not repeat the general logic, leading to Ward identities like (1.2) in DF and network matrix models.

\subsection{The main theme}

Screening charge $\hat{Q}$, acting on the Fock space $\mathcal{F}_{\alpha}=\left\{\operatorname{Pols}\left(\tau_{n}\right)\right\} \cdot e^{\alpha T_{0}}$, is

$$
\hat{Q}=\oint \hat{S}(x) d x=\operatorname{res}_{x=0} \hat{S}(x)
$$




$$
\hat{S}(x)=: e^{\sqrt{2} \hat{\phi}(x)}:=\underbrace{\exp \left(\sum_{n>0} \frac{\tau_{n} x^{n}}{n}\right)}_{\sum_{n} x^{n} \chi_{n}\{\tau\}} e^{T_{0}} x^{2 \partial_{0}} \exp \left(-\sum_{n>0} \frac{2}{x^{n}} \frac{\partial}{\partial \tau_{n}}\right)
$$

where $\chi_{n}\{\tau\}$ are the characters of symmetric representations $[n]$ of $s l$ algebras (the Schur polynomials in this particular case). Applied to a highest-weight state (i.e. the one annihilated by all negative modes $\hat{a}_{-n}=-\sqrt{2} n \frac{\partial}{\partial \tau_{n}}$ ) with negative half- integer $\alpha$

$$
|m+1\rangle=e^{-\frac{1}{2}(m+1) T_{0}}
$$

it gives

$$
\hat{Q}|m+1\rangle=\chi_{m}\{\tau\}|m-1\rangle
$$

Residue is non-vanishing, because $x^{2 \partial_{0}}$ converts $\mid m+1>$ into $x^{-m-1}$. Similarly

$$
\hat{Q}^{2}|m+2\rangle=\chi_{[m m]}\{\tau\}|m-2\rangle
$$

where the calculation involves

$$
-\frac{1}{2} \sum_{m_{1}, m_{2}} \chi_{m_{1}} \chi_{m_{2}} \oint \oint d x_{1} d x_{2} x_{1}^{m_{1}-m-2} x_{2}^{m_{2}-m-2}\left(x_{1}-x_{2}\right)^{2}=\chi_{m}^{2}-\chi_{m+1} \chi_{m-1}=\chi_{[m, m]}
$$

and

$$
\hat{Q}^{r}|m+r\rangle=\chi_{\left[m^{r}\right]}\{\tau\}|m-r\rangle
$$

i.e. the power of $\hat{Q}$ acts as a character of rectangular Young diagram. This is the old result by [98-104]. The rectangular diagrams arise from the Cauchy formula

$$
\prod_{i=1}^{r} \exp \left(\sum_{n>0} \frac{\tau_{n} x_{i}^{n}}{n}\right)=\exp \left(\sum_{n>0} \frac{1}{n} \tau_{n} \sum_{i=1}^{r} x_{i}^{n}\right)=\sum_{\lambda} \chi_{\lambda}\{\tau\} \chi_{\lambda}[\vec{x}]
$$

with a sum over all Young diagrams $\lambda$ (actually, with no more than $r$ lines) after the Vandermonde projection

$$
\prod_{i=1}^{r} \oint \frac{d x_{i}}{x_{i}^{m+r}} \Delta(\vec{x})^{2} \chi_{\lambda}[\vec{x}] \sim \delta_{\lambda,\left[m^{r}\right]}
$$

which is a direct generalization of (2.5).

Since the screening charge commutes

$$
\left[\hat{L}_{n}, \hat{Q}\right]=0
$$

with the Virasoro generators

$$
\hat{L}_{n}=\sum_{k}(k+n) \tau_{k} \frac{\partial}{\partial \tau_{k+n}}+\sum_{k=1}^{n-1} k(n-k) \frac{\partial^{2}}{\partial \tau_{k} \partial \tau_{n-k}}+2 n \frac{\partial^{2}}{\partial \tau_{n} \partial T_{0}}, \quad n>0
$$


one has

$$
\hat{L}_{n} \hat{Q}^{r}|m+r\rangle=\hat{Q}^{r} \hat{L}_{n}|m+r\rangle=0 \text { for } n>0
$$

In application to (2.6), this gives

$$
\hat{L}_{n} \chi_{\left[m^{r}\right]}=n(m-r) \frac{\partial \chi_{\left[m^{r}\right]}}{\partial \tau_{n}} \quad n>0
$$

while the action of

$$
\hat{L}_{0}=\sum_{k} k \tau_{k} \frac{\partial}{\partial \tau_{k}}+\frac{\partial^{2}}{\partial T_{0} \partial T_{0}}
$$

gives just the size of the Young diagram:

$$
\hat{L}_{0} \chi_{\left[m^{r}\right]}=m r \cdot \chi_{\left[m^{r}\right]}
$$

In the Miwa parametrization $\tau_{n}=\sum_{i} X_{i}^{n}$, this turns into the statement about the Calogero eigenfunctions. Also $Q^{r}|m+r\rangle$ are singular vectors in Verma modules and (2.12) can be considered as the simplest version of $\mathrm{BPZ}$ equations for correlators with degenerate fields, [12].

Equation (2.12) provides a simple example of the Ward identity for the state $\hat{Q}^{r} \mid m+$ $r>$, which can be promoted to identity for the matrix element in conformal field theory, i.e. in the abstract Fock module and corresponding Sugawara energy-momentum tensor (which we denote by Gothic letters), $\chi_{\left[m^{r}\right]}=<m-r\left|\hat{C} \mathcal{T}(z) \hat{\mathcal{Q}}^{r}\right| m+r>$ by additional insertion of the intertwining operator, see below. We are now ready to formulate the main theme of the present paper:

A trivial symmetry property (2.9) gives rise to a non-trivial equation for the matrix element (2.12), provided one can calculate (2.6).

In what follows we extend this simple example to matrix elements of an arbitrary network of intertwining operators, what allows to reveal in a rather explicit form the hidden DIM symmetry of the Seiberg-Witten/Nekrasov theory.

We continue in this section with variations on the main theme, developing it at conceptual level. Next sections will describe technical details of the story.

\subsection{Variation I: matrix elements in the free-field theory}

Actually, in theory of free field $\phi(z)$, the bra vacuum state is annihilated by all the negative mode operators $\hat{a}_{-n}=\tau_{n} / \sqrt{2}, n>0$, i.e. contains $\prod_{n>0} \delta\left(a_{-n}\right)$ in holomorphic representation. Thus, one can not simply convert (2.6) into a statement that $\chi_{\left[m^{r}\right]}\{\tau\}$ is equal to $<m-r\left|Q^{r}\right| m+r>$ : this matrix element would not depend on $\tau$ at all. The way out is to introduce a special intertwining operator

$$
\hat{C}\{p\}=\exp \left(\sum_{n>0} \frac{p_{n} \hat{a}_{n}}{n}\right)
$$

which converts the bra vacuum into the coherent state

$$
\langle m| \longrightarrow\langle m| \hat{C}\{p\}
$$


with the property

$$
\langle m| \hat{C}\{p\} \hat{a}_{-n}=p_{n} \cdot\langle m| \hat{C}\{p\}
$$

This allows us to rewrite (2.6) as

$$
\chi_{\left[m^{r}\right]}\left\{\sqrt{2} p_{n}\right\}=\left\langle m-r\left|\hat{C}\{p\} \hat{\mathcal{Q}}^{r}\right| m+r\right\rangle
$$

Among many complications as compared with (2.6), there is $\sqrt{2}$, which reflects the fact that the character is extracted here from the screening charge in a single field ("current") realization. A more adequate kind of formulas arise within the fermionic realization (see section 3.2 of [72] and section 2.6 below) which involves two scalar fields, and $\sqrt{2}$ is a result of basis rotation to their symmetric combination.

\subsection{Variation II: generating functions}

We can make from particular Virasoro generators $\hat{L}_{n}$ a single operator (stress tensor)

$$
\hat{T}(z)=\sum_{n \in \mathbb{Z}} \frac{\hat{L}_{n}}{z^{n+2}}
$$

Positive and zero modes with $n \geq 0$ are given by (2.10) and (2.13) respectively, negative modes are:

$$
\hat{L}_{-n}=\sum_{k} k \tau_{k+n} \frac{\partial}{\partial \tau_{k}}+\tau_{n} \frac{\partial}{\partial T_{0}}+\frac{1}{4} \sum_{k=1}^{n-1} \tau_{k} \tau_{n-k}
$$

so that

$$
\left[\hat{L}_{n}, \hat{L}_{m}\right]=(n-m) \hat{L}_{n+m}+\frac{n\left(n^{2}-1\right)}{12} \delta_{n+m, 0}
$$

for the properly regularized sum $\sum_{n>0} n=-\frac{1}{12}$.

Symmetry (2.9) actually holds for all $n \in Z$.

We will also need a "current"

$$
\begin{aligned}
\hat{J}(z) & =\partial_{z} \hat{\phi}(z)=\sum_{n \in Z} \frac{\hat{J}_{n}}{z^{n+1}} \\
\text { with } \quad \hat{J}_{-n} & =\frac{\tau_{n}}{\sqrt{2}}, \quad \hat{J}_{0}=\sqrt{2} \frac{\partial}{\partial T_{0}}, \quad \hat{J}_{n}=\sqrt{2} n \frac{\partial}{\partial \tau_{n}}
\end{aligned}
$$

and

$$
\begin{aligned}
{\left[\hat{J}_{n}, \hat{J}_{m}\right] } & =n \delta_{n+m, 0} \\
{\left[\hat{L}_{n}, \hat{J}_{m}\right] } & =-m J_{n+m}
\end{aligned}
$$

The two operators are related by the Sugawara relation

$$
\hat{T}(z)=: \frac{1}{2} \hat{J}(z)^{2}:
$$

where normal ordering puts all $p$-derivatives to the right of all $p$ 's (in each term of the formal series). 
The generating functions satisfy the commutation relations

$$
\begin{aligned}
{[\hat{J}(z), \hat{J}(w)] } & =\delta^{\prime}(w / z) \\
\delta(x) & =\sum_{n \in \mathbb{Z}} x^{n}
\end{aligned}
$$

In terms of generating functions, the Ward identity (2.12), i.e. the corollary of symmetry (2.9) becomes

$$
\left[z^{2} \hat{T}(z)-\frac{m-r}{\sqrt{2}} z \hat{J}(z)\right]_{-} \cdot \chi_{\left[m^{r}\right]}=0
$$

or, in other words, a regularity constraint

$$
\left(z^{2} \hat{T}(z)-\frac{m-r}{\sqrt{2}} z \hat{J}(z)\right) \cdot \chi_{\left[m^{r}\right]}=\operatorname{Pol}(z)
$$

This will be the typical form of Ward identities (regularity condition for $q q$-characters) for network Nekrasov functions $Z$ generalizing the simple character $\chi_{\left[m^{r}\right]}$.

\subsection{Variation III: DF model}

Expressions (2.6) and (2.1) together imply the integral representation of the matrix element

$$
\begin{aligned}
\chi_{\left[m^{r}\right]}\{\tau\} & =\left\langle m-r\left|\hat{C}\left\{\tau_{n} / \sqrt{2}\right\} \hat{\mathcal{Q}}^{r}\right| m+r\right\rangle \\
& =-\frac{1}{r !} \underbrace{\oint \ldots \oint}_{r}\left(\prod_{i=1}^{r} \frac{\mathcal{G}\left\{\tau \mid x_{i}\right\} d x_{i}}{x_{i}^{m+r}}\right) \prod_{i<j}\left(x_{i}-x_{j}\right)^{2}=\langle 1\rangle_{\mathrm{DF}_{\mathrm{m}, \mathrm{r}}} \\
\mathcal{G}\{\tau \mid x\} & =\exp \left(\sum_{n=1}^{\infty} \frac{\tau_{n} x^{n}}{n}\right)=\sum_{n=0}^{\infty} x^{n} \chi_{n}\{\tau\}
\end{aligned}
$$

which is the archetypical example of DF or conformal matrix model [70-72, 102-104].

Ward identity (2.27), which is a trivial corollary of commutativity (2.9) looks now like a not-so-obvious set of integral identities:

$$
\begin{aligned}
& \left(z^{2} \hat{T}(z)-\frac{m-r}{\sqrt{2}} z \hat{J}(z)\right)\langle 1\rangle_{\mathrm{DF}_{\mathrm{m}, \mathrm{r}}} \\
& \quad=\left\langle\sum_{k, i} \frac{\tau_{k} x_{i}^{k+1}}{z-x_{i}}+\sum_{i, j=1}^{r} \frac{x_{i} x_{j}}{\left(z-x_{i}\right)\left(z-x_{j}\right)}-(m-r) \sum_{i} \frac{x_{i}}{\left(z-x_{i}\right)}\right\rangle_{\mathrm{DF}_{\mathrm{m}, \mathrm{r}}}=\operatorname{Pol}(z)
\end{aligned}
$$

Actually there are two standard ways to derive the 1.h.s.:

(1) by using bosonization, which is the simplest version of free-field (FF) formalism, i.e. the Wick rule for decomposition of correlators into pair ones,

$$
\hat{T}(z)\langle 1\rangle_{\mathrm{DF}_{\mathrm{m}, \mathrm{r}}}=\langle\hat{C}\left(\tau_{n} / \sqrt{2}\right) \cdot \underbrace{\mathcal{T}(z)}_{\frac{1}{2}: \partial \varphi(z)^{2}:} \cdot \prod_{i=1}^{r} \oint e^{\sqrt{2} \varphi\left(x_{i}\right)} d x_{i}\rangle_{\mathrm{FF}_{\mathrm{m}}}
$$

where the index $m$ refers to a special way of handling the zero mode of $\varphi$ and $\varphi(z)$ refers to the scalar field acting in the abstract Fock module, and 
(2) by a change of integration variables $\delta x_{i}=\epsilon x_{i}^{n+1}$ in the multiple integral (2.28), [17, $18,73-79]$ : in this case we get the identities in a slightly different form:

$$
\left\langle\sum_{i<j}^{r} 2 \frac{x_{i}^{n+1}-x_{j}^{n+1}}{x_{i}-x_{j}}+\sum_{i, k} \tau_{k} x_{i}^{k+n}+\sum_{i}^{r}(n+1-m-r) x_{i}^{n}\right\rangle_{\mathrm{DF}_{\mathrm{m}, \mathrm{r}}}=0, \quad n>0
$$

In this paper we actually need an outdated and tedious third way:

(3) the operator formalism based on an explicit calculation of commutators arising when the stress tensor is carried from the left to the right through the screening operators: this is what we are now doing, starting from section 2.0 and this is what in the simplest case brought us to the Ward identity in the form (2.29).

Both the OPE-based and change-of-integration-variables/total-derivative approaches should also work in the network model context, but they still need to be developed.

\subsection{Variation IV: multi-field case}

The network matrix models can be considered as associated with networks of branes (branewebs [105-110]), which being projected onto the $4-5$ plane look like segments with different slopes. From the point of view of Yang-Mills theories, interpretation of the different slopes is different. Surprisingly or not, it is also different at the present level of understanding of the DIM symmetry. Throughout the section, we distinguish only between the horizontal and vertical segments, while intermediate slopes appear in this section only in sections 2.8 and 2.9. Our next variations introduce and describe the associated notions.

The first one is horizontal branes. These are associated with different free fields. Generalization of the DF model to $K$-field case provides $\mathcal{W}_{K}$ constraints for models with $K$ horizontal branes. An additional procedure can be applied to separate a "center-ofmass" field: this explains why in the previous subsection 2.3 the number of fields was one rather than two.

The multi-field conformal model [72] is defined as

$$
\left\langle\vec{m}-\vec{r}\left|\prod_{a=1}^{K-1} \hat{C}_{a}\left\{\tau_{n}^{(a)} / \sqrt{2}\right\} \hat{\mathcal{Q}}_{a}^{r_{a}}\right| \vec{m}+\vec{r}\right\rangle=\langle 1\rangle_{\mathrm{DF}_{\vec{m}, \vec{r}}}
$$

where the screening charges now carry additional indices labeled by $K-1$ simple roots $\vec{\alpha}_{a}$ of $s l_{K}$. They are actually associated with segments of the vertical branes ending on two adjacent horizontal branes, figure 3 , a), in accordance with the decomposition $\vec{\alpha}_{a}=$ $\vec{e}_{a+1}-\vec{e}_{a}$. In other words, a better labeling of $\mathcal{Q}$ is by pairs of indices $a b$, each corresponding to a particular horizontal (in fact, any non-vertical, see section 2.5) brane. ${ }^{1}$ Now the matrix model partition function depends on $K$ sets of times, one of which is associated with the "center of mass" and actually decouples in the DF model (2.28), thus it was actually suppressed in that formula. However, this is not always true: the decoupling will not take

\footnotetext{
${ }^{1}$ To avoid possible confusion, note that in [50] an "orthogonal" labeling rule was used, treating horizontal edges of the network as segments between the vertical ones.
} 
place already in the Chern-Simons deformation of (2.28) in section 2.5, and all the $\mathcal{M}$ sets of times will be relevant in generic DIM considerations. This phenomenon is familiar in the CFT approach to Nekrasov functions, where relevant is the Heis + Virasoro symmetry and its generalizations rather than the Virasoro alone. This is also reflected in appearance of " 1 " in the popular notation $\mathcal{W}_{1+\infty}$.

Algebraically, the multi-field generalization is controlled by the comultiplication $\Delta_{D I M}$, which builds all the symmetry generators from a single element of DIM:

current algebra
$\downarrow$
Virasoro
$\downarrow$
$\mathcal{W}_{3}$
$\downarrow$
$\cdots$
$\downarrow$
$\mathcal{W}_{K}$
$\downarrow$
$\cdots$

This comultiplication adds new scalar fields, and non-linearity of the usual $4 d$ Sugawara formulas is mostly due to elimination of the center-of-mass field; what makes this possible is the exponential form of symmetry generators beyond $4 d$. Somewhat symbolically, the Sugawara formulas for the stress tensor (at the second level of DIM) arise from the expansion of characters (in fact, $q$-characters)

$$
\begin{aligned}
& K=2: \quad \mathcal{T}_{s l_{2}}=\frac{1}{2}\left(e^{J}+\underline{e^{-J}}\right)=1+\frac{1}{2} J^{2}+\ldots \\
& K=3: \quad \mathcal{T}_{\text {sl }}=\frac{1}{3}\left(e^{J_{1}}+e^{J_{2}-J_{1}}+\underline{e^{-J_{2}}}\right)=1+\frac{1}{3}\left(J_{1}^{2}-J_{1} J_{2}+J_{2}^{2}\right)+\ldots \\
& K: \quad \mathcal{T}_{s l_{K}}=1+\frac{1}{K !} \sum_{a, b=1}^{K-1} C_{a b} J_{a} J_{b}+\ldots \\
& K=\infty: \quad \mathcal{T}_{s l_{\infty}}=1+\text { const } \cdot \int(\nabla J)^{2}+\ldots
\end{aligned}
$$

underlined in the first two lines are terms appearing due to the center-of-mass reduction

$$
\sum_{a=1}^{K} J_{a}=0
$$

$C_{a b}$ is the Cartan matrix for $s l_{K}$, which the $K=\infty$ limit describes a difference Laplace operator $\nabla^{2}$. Other $\mathcal{W}$-operators made from higher powers of $J$ arise in the same way at 
higher levels of DIM, i.e. after several applications of the comultiplication $\Delta_{D I M}$, e.g. at $K=3$ the second generator of the $W_{3}$-algebra is

$$
\mathcal{W}_{s l_{3}}^{(3)}=\frac{1}{3}\left(e^{-J_{1}}+e^{J_{1}-J_{2}}+\underline{e^{J_{2}}}\right)
$$

so that the standard $W_{3}$-generator is a difference

$$
\mathcal{T}_{s l_{3}}-\mathcal{W}_{s l_{3}}^{(3)} \sim \frac{1}{3} J_{1} J_{2}\left(J_{1}-J_{2}\right)+\ldots
$$

\subsection{Variation V: Chern-Simons (CS) model}

The brane slopes show up in a specially designed $4 d$ limit as additional square-logarithmic terms $\left(\log x_{i}\right)^{2}$ in the action of the DF matrix model (2.28), giving rise to what is often called the CS matrix model [111-124]:

$$
\langle 1\rangle_{\mathrm{CS}_{\mathrm{r}}}=\frac{1}{r !} \prod_{i=1}^{r} \oint d x_{i} \mathcal{G}\left\{\tau \mid x_{i}\right\} e^{\gamma\left(\log x_{i}\right)^{2}} \prod_{i<j}\left(x_{i}-x_{j}\right)^{2}
$$

The parameter $\gamma$ controls the brane slope, it vanishes for the horizontal branes, while for the vertical ones it becomes infinite and the story gets a separate twist, see section 2.6 below.

From the point of view of DIM symmetry of the network model, the Virasoro/Ward constraints should look similar with and without these logarithmic terms, in the sense that they should be always dictated by the Wick theorem hidden in the algebraic structures of DIM. There is, however, a crucial difference: in this case, the U(1)-mode should not decouple for non-trivial slopes, and two sets of times survive (see section 2.6). This is reflected in the fact that one needs to consider $\mathcal{G}\{\tau \mid x\}$ depending on $\tau_{n>0}$ and $\tau_{n<0}$ in (2.38),

$$
\mathcal{G}\{\tau \mid x\}=\exp \left(\sum_{n \in \mathbb{Z}}^{\infty} \frac{\tau_{n} x^{n}}{n}\right)
$$

in order to construct the Ward identities. Then, a counterpart of (2.31) for (2.38) looks somewhat different $[122,123,125-127]$ :

$$
\left\langle(n-r+1) \sum_{i}^{r} x_{i}^{n}+\sum_{i}^{r} x_{i}^{n}\left(\log \theta\left(x_{i} \mid q\right)\right)^{\prime}+2 \sum_{i<j} \frac{x_{i}^{n+1}-x_{j}^{n+1}}{x_{i}-x_{j}}+\sum_{k, i} \tau_{k} x_{i}^{n+k}\right\rangle=0
$$

where $q=\exp \left(\frac{1}{2 \gamma}\right)$ and $\theta(x \mid q)=\sum_{\nu=-\infty}^{\infty} q^{\nu^{2} / 2} x^{\nu}$.

\subsection{Variation VI: correlators with vertex operators}

The vertical branes are associated with insertions of vertex operators into the DF and CS models. A particular instance of the vertex operator is the screening current. As already mentioned in section 2.4, screening charges are segments of vertical branes between the two neighbour horizontal ones, and they can be considered as contractions of two vertex operators attached to these two branes. However, the relevant operators are special, 
namely, they are $e^{\alpha \phi}$ with $\alpha= \pm 1$ : a kind of "fermion vertices" (in fact, intertwining operators) $\psi^{ \pm}=e^{ \pm \phi}$. Accordingly, the screening charges should be associated with bilinears $\psi_{a}^{+}(x) \psi_{b}^{-}(x)$, "non-local" in the vertical direction:

$$
\hat{Q}_{a b}=\oint \psi_{a}^{+}(x) \psi_{b}^{-}(x) d x
$$

This non-locality explains, among other things, why the screening currents are "naturally" exponentials rather than $\partial \phi$-like currents, as well as the emergency of peculiar $\sqrt{2}$ in (2.1) coming from the $45^{\circ}$ rotation of the basis $\phi_{1}, \phi_{2}$ into $\frac{\phi_{1} \pm \phi_{2}}{\sqrt{2}}$.

In general, fermion operators (peculiar intertwiners in DIM) carry a Young-diagram label $\lambda$ instead of $x$ and the screening charge is a convolution of these indices (see s.3.2 of [72] for details). Interchanging of + and - labels changes the screening charge to the dual one (in algebraic terms, this corresponds to using instead of a positive root the corresponding negative one): as usual in conformal matrix models [70-72], the use of dual charges is unnecessary. In fact, one can connect every screening charge with a simple root: one can associate with each end of leg $a$ a basis vector $\vec{e}_{a}$, then, the screening charge $Q_{a, a+1}$ corresponds to a simple root $\vec{\alpha}_{a}=\vec{e}_{a+1}-\vec{e}_{a}$.

In operator formalism the correlator of vertex operators is just a matrix element of an ordinary product of linear operators. A generic vertex operator is constructed from the primary field $V_{\alpha}(x)$ and is labeled by the Young diagram $\lambda$ :

$$
\hat{V}_{\alpha}^{\lambda}=\hat{L}_{-\lambda} V_{\alpha}(x)
$$

with $\hat{L}_{-\lambda}=\prod_{i} \hat{L}_{-\lambda_{i}}$. The conjugation with $\hat{L}_{-1}$ moves it to an arbitrary point $z$ :

$$
\hat{V}_{\alpha}^{\lambda}(x+z)=e^{z \hat{L}_{-1}} \hat{V}_{\alpha}^{\lambda}(x) e^{-z \hat{L}_{1}}
$$

However, in CFT the positions of operators does not matter: they can be considered as located at points in the complex $z$-plane, or, more generally, on a Riemann surface (in the latter case same traces need to be taken in operator formalism).

Still, location of the stress-tensor insertion does matter: in the Riemann surface picture, it is associated with a choice of a contour encircling the vertex operator insertions, and correlator depends on the homology class of this contour. Changing the class is equivalent to commutation of $T(z)$ with the vertex operator, which is read off the commutation relations

$$
\left[L_{n}, V_{\alpha}(x)\right]=x^{n+1} V_{\alpha}^{\prime}(x)+\alpha^{2}(n+1) x^{n} V_{\alpha}(x)
$$

and those of the Virasoro algebra. This is what we did in the derivation of (1.5) placing the stress-tensor to the left, and to the right of vertex operators.

Central-charge-preserving comultiplication $\boldsymbol{\Delta}_{M S}$. The action of Virasoro algebra is provided by the Moore-Seiberg comultiplication $\Delta_{M S}$, which is given by the ordinary Leibnitz rule on the negative modes $T_{-}$, but the positive modes act differently:

$$
\Delta_{M S}\left(L_{n}\right) R_{1} \otimes R_{2}=\left(\sum_{k=0}^{\infty} z^{n+1-k}\left(\begin{array}{c}
n+1 \\
k
\end{array}\right) L_{k-1} R_{1}\right) \otimes R_{2}+R_{1} \otimes L_{n} R_{2}
$$


This comultiplication can be read off the conformal Ward identities, [128] and celebrates two important properties:

- It is parameterized by an arbitrary parameter $z$,

- it does not change the central charge, in contrast with the comultiplication in the DIM algebra that we use below.

\subsection{Variation VII: Nekrasov functions}

We define the Nekrasov function as partition function of the DF/CS network matrix model depending on parameters $\vec{\alpha}_{i}, z_{i}$ and $N_{a}$, associated respectively with external legs (assumed vertical), horizontal and vertical edges of the graph $\Gamma$ : schematically,

$$
Z_{\Gamma}=\left\langle\prod_{i=1}^{4} \hat{V}_{\vec{\alpha}_{i}}\left(z_{i}\right) \exp \left(\sum_{a=1}^{K-1} \hat{Q}_{a, a+1}\right)\right\rangle_{\mathrm{DF}_{N_{a}}}
$$

and this partition function describes the $A_{1}$-quiver with obvious modifications for more sophisticated quivers, [50] (changing the number of vertex operators and adding more screening charges that differ by the choice of the integration contours). The right numbers of screening charges are automatically selected from the series expansion of the exponential by zero mode conditions.

On the gauge theory side, this data describes the theory with the gauge group $\mathrm{SU}(K)$ and $2 K$ fundamental matter hypermultiplets (i.e. zero $\beta$-function). Here the numbers $N_{a}$ are the Coulomb moduli, the hypermultiplet masses are parameterized by the vertex operator parameters $\vec{\alpha}_{i}$ and the positions of vertices (rather their double-ratio) control the instanton expansion in the gauge theory. Note that this theory is characterized by zero $\beta$-function, all other cases are obtained by evident degeneration. The case of adjoint matter hypermultiplets is described by the elliptic DIM algebras ${ }^{2}$ [140-143] and is out of scope of the present paper. The other quiver theories, say $A_{k}$ are described, on the physical side, by a product of $k$ gauge groups: $\prod_{i}^{k} \mathrm{SU}\left(n_{i}\right)$ with $\kappa_{i}=2 n_{i}-n_{i-1}-n_{i+1}$ bifundamental hypermultiplets for each $i$ transforming under the gauge groups $\mathrm{SU}\left(n_{i}\right)$ and $\mathrm{SU}\left(n_{i+1}\right)$. There are also $\kappa_{0}$ and $\kappa_{k}$ fundamental hypermultiplets that are transformed under $\operatorname{SU}\left(n_{1}\right)$ or $\mathrm{SU}\left(n_{k}\right)$ (we put $n_{0}=n_{k+1}=0$ ). These theories have also zero $\beta$-functions, other cases can be obtained by a degeneration of hypermultiplet masses. Note that the Nekrasov network partition functions typically contain additional singlet fields, which corresponds to $\mathrm{U}(K)$ instead of $\mathrm{SU}(K)$ group. The contribution of this singlet factorizes out and reduces just to a simple multiplier in the Nekrasov function.

While exponentiation of bosonized screenings $Q=\oint e^{\phi}$ can look somewhat artificial, the same procedure is very natural in the fermionic version $Q=\oint \psi^{+} \psi^{-}$: this adds $\psi$ bilinear terms to the free fermion action, i.e. leaves it quadratic. This is the reason for integrability, and in bosonized version this is reflected in integrable properties of Toda like systems with exponential actions.

Exponentiation of fermionic screenings makes a new interesting twist after the $q$ deformation in section 2.10, see eq. (2.50) below.

\footnotetext{
${ }^{2}$ By DIM algebras in this paper we mean both DIM and its limits like affine Yangian [49, 129-139].
} 


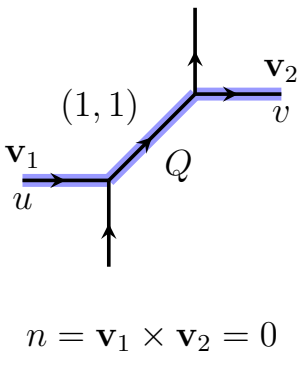

a)

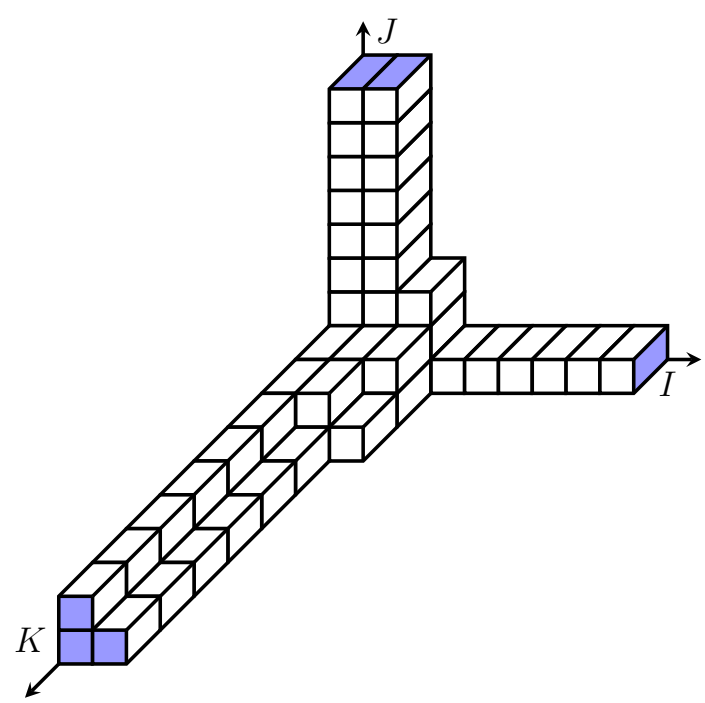

b)

Figure 5. a) Simplest toric diagram. The intermediate edge has slope $(1,1)$ and length $Q$ and framing factor $n=\mathbf{v}_{1} \times \mathbf{v}_{2}=0$. The "horizontal" line with spectral parameters $u$ and $v$ is shown in blue. The length of the intermediate edge is determined by the ratio of the spectral parameters on the adjacent edges, $Q=\frac{v}{u}$. b) An example of a 3d Young diagram which contributes to the vertex $C_{[1],[2],[2,1]}$. The vertex $C_{[1],[2],[2,1]}$ is given by the weighted sum over all $3 \mathrm{~d}$ Young diagrams with three fixed asymptotics shown in blue.

\subsection{Variation VIII: network model level. Network as a Feynman diagram}

Network model is defined for a planar 3-valent graph $\Gamma$ with edges parameterized by slopes and lengths. Slopes are given by pairs of numbers $\left(X_{1}, X_{2}\right)$, see figure 5 , and lengths by parameters $Q$. The 2 -component vectors $\vec{X}$ are conserved at the vertices of $\Gamma: \vec{X}_{v}{ }^{\prime}+\vec{X}_{v}{ }^{\prime \prime}+$ $\vec{X}_{v}^{\prime \prime \prime}=0$ at each vertex $v$; this is a stability condition for the brane-web. The graph $\Gamma$ with this structure describes a $l a[3,4]$ the tropical spectral curve of the underlying integrable system, but for our purposes it can be considered just as a Feynman diagram with cubic vertices and momenta $Q \vec{X}$ on the edges, associated with some effective Chern-Simons-type field theory. Expressions $Z_{\Gamma}$ for this Feynman diagram (Nekrasov partition function or generalized conformal block) is build by convolution of vertices $C_{I J K}\left(\vec{X}^{\prime}, \vec{X}^{\prime \prime}, \vec{X}^{\prime \prime \prime} \mid q\right)$ and propagators $\Pi^{I J}(Q)$, where indices $I, J, K$ are Young diagrams, and $C_{I J K}$ are, in turn, "(refined) topological vertices" [35-37] given [144-148] by sums over $3 d$ (plane) partitions with three boundary conditions described by three ordinary Young diagrams $I, J, K$, see figure $5, \mathrm{~b}$ ).

In the generic network matrix model, the exponentials of screening charges no longer turn into exponential of "fermions": it produces an elementary 3 -valent vertex (=refined topological vertex) providing the true DIM intertwiner. Automatic is now not only adjustment of the number of screenings, but also matching between their $\psi^{+}$and $\psi^{-}$constituents.

- Screening charges are substituted by vertical lines between pairs of horizontal brains, $\oint \exp \left(\vec{\alpha}_{i j} \vec{\phi}\right)$, involving two free fields associated with the corresponding branes. 
- Slopes of the horizontal branes enter the matrix model description through $\left(\log x_{i}\right)^{2}$ terms in the action, see (3.45) in s.3. The coefficient is made out of the skew product (see figure 5 a))

$$
\vec{X}_{v_{1}} \wedge \vec{X}_{v_{2}}
$$

where $\vec{X}_{v_{1}}, \vec{X}_{v_{2}}$ are associated with the external horizontal lines, one incoming, the other one outgoing. In the case with several horizontal lines, see e.g. (3.42), one has to consider $\vec{X}_{v_{1}}, \vec{X}_{v_{2}}$ for different horizontal lines, and the answer in this case does not depend on the concrete choice of these lines.

We described in this subsection a generic network model. One can consider its particular case: the model that gives rise to the quiver gauge theory (as described in the previous subsection). In this case (for any quiver gauge theory), one can construct a K-theoretic version of the Nekrasov functions, $Z_{\Gamma},[149-152]$. They coincides [36, 37, 153, 154] with the refined partition functions in the corresponding geometry, which can be constructed via the refined topological vertex.

Another possibility is to consider the quiver theories with zero $\beta$-functions (so that all other can be obtained via various limiting procedures from these) and all gauge groups coinciding, $n_{i}=n \forall i$. These theories are associated with so called balanced networks and can be immediately described within the representation theory of DIM algebras, and the requirement of all gauge groups having the same rank is implied by a possibility of immediate extension of DIM to the elliptic DIM: this latter describes the quiver gauge theories with adjoint matter, where the condition $n_{i}=n$ is inevitable. We discuss the issue of balanced networks in the next subsection.

\subsection{Variation IX: balanced network model}

As usual, the $q, t$-deformation leads to overloaded formulas, but in fact it drastically simplifies them by providing a very clear and transparent interpretations and unifying seemingly different ingredients. Namely, everything gets controlled by the DIM symmetry: the edges of graph carry DIM representations, the topological vertices $C$ become their intertwiners, and symmetries (stress-tensor and its $\mathcal{W}$-counterparts) are just the generators of DIM acting in tensor products of representations and thus defined by powers of the comultiplication $\Delta_{D I M}$ (which is different from $\Delta_{M S}$ ).

An exhaustive description of the network models depends on development of representation theory for the double affine algebra DIM, and it is not yet brought to the generality level of [95-97] for ordinary affine algebras. In particular, at the moment, it is not immediate to describe within the DIM framework an arbitrary DF or CS matrix model. However, among the DF matrix models there is a subclass that is directly lifted to rather peculiar networks, which we call balanced which are controlled by an analogue of the level one representations of Kac-Moody algebras and allow a drastically simplified bosonization and even fermionization. As we already mentioned the balanced networks correspond to special quiver gauge theories with zero $\beta$-functions. 
We provide the details in section 3 below, and devote the rest of this subsection to a bird's eye view survey which makes use of an oversimplified, almost symbolic notation. One can find the exact formulas in section 3.

The network basically is a constructor with the main building block being a (refined) topological vertex, which is a matrix element of an intertwining operator that intertwines three representations, hence, the topological vertex is associated with three legs.

The balanced network is defined by three requirements:

(a) Consider a class of representations of DIM such that each leg is parameterized by a pair of integers $\vec{M}=\left(M_{1}, M_{2}\right)$ (DIM central charges) and a Young diagram $Y$. Then, the integers are subject to the conditions: $\sum_{i}^{3} \vec{M}_{i}=0$ and $\left|\vec{M}_{i} \wedge \vec{M}_{j}\right|=1$ for any pair of legs in the vertex. ${ }^{3}$ As in section 2.8 , we associate every vector $\left(M_{1}, M_{2}\right)$ with an edge of the network, and parameterize slopes as ratios $M_{2} / M_{1}$.

(b) Assume one of the legs of vertices is always vertical, $\vec{M}=(0, M)$. This implies that $M= \pm 1$ and that the two other vertices are $( \pm 1, L \pm 1)$. From a general network with rational slopes one can make this minimal (i.e. that with all vertices having a vertical edge) by a sequence of resolutions, introducing new edges and triple vertices. Reversing, a general rational network arises from a minimal one, when some edges are shrunk to a point while others "fattened" (i.e. described by $M_{1}$ and $M_{2}$ which are not coprime, this can be needed to keep vertices three-valent).

We represent such a minimal rational network (figure 4 , a)) by a set of $K$ horizontal lines connected by vertical segments (for planar graph, only adjacent lines can be connected), which can also be as external vertical legs (to the lowest and highest horizontal lines). Horizontal segments are also labeled by slopes: in other words, we draw all non-vertical edges horizontal, but keep the slopes as labels.

(c) Balanced is the minimal rational network where all external legs are either vertical or horizontal, i.e. either $( \pm 1,0)$ or $(0, \pm 1)$.

Partition functions for non-balanced networks have singular limit $t \longrightarrow 1, q / t=$ fixed and thus do not directly reduce to a DF model in $4 d$. Also the $\mathrm{U}(1)$ center-of-mass field does not split from the Virasoro and other symmetries in this case. However, maybe not these two issues are the main drawbacks, the real problem is a more sophisticated representation theory needed to lift any of the three above restrictions: balance, minimality and rationality (in the order of complexity).

From now on, we draw all networks on the square lattice: the vertical lines $(0,1)$ are vertical, while all the lines with slopes $(1, M)$ are horizontal and just carry the charges $(1, M)$.

The partition function for the balanced network is a contraction of just two types of vertices: the generalized "fermions" $\Psi$ and $\Psi^{*}$, which intertwine the DIM representations: $(1, M) \otimes(0,1) \longrightarrow(1, M+1)$ and $(1, M+1) \otimes(0,-1) \longrightarrow(1, M)$. These intertwiners can be described in terms of free field, which acts as an operator in "horizontal" direction, i.e. converts the Verma module $V_{(1, M)}$ into $V_{(1, M \pm 1)}$. Thus, of the three Young diagrams

\footnotetext{
${ }^{3}$ In terms of topological strings, these are the Calabi-Yau and smoothness conditions.
} 
$\Psi$ depends explicitly only on one: that sitting on the vertical leg, while those on two other, horizontal legs parameterize the states in the Fock space, but not the operator. Instead $\Psi$ depends on the spectral parameter $u$, as well as on the position. The position is described by a continuous coordinate $z$ along the horizontal line and by the discrete number $a$ labelling the horizontal line itself. Actually, all $\Psi_{a}$ and $\Psi_{a}^{*}$ with a given $a$ depend on the free field $\phi_{a}$ : there are $K$ independent free fields for $K$ horizontal lines. It remains to provide explicit formulas for the $\Psi$-operators, slightly symbolically

$$
\prod_{I} \Psi_{\lambda_{I}}\left[z_{I}\right] \prod_{J} \Psi_{\mu_{J}}^{*}\left[z_{J}^{*}\right] \longrightarrow \prod_{I, J} \exp \left(\sum_{n \neq 0} \frac{1}{n}\left(\omega^{|n|}\left[\lambda_{I}, z_{I}\right]_{n} \mathfrak{a}_{n}-\left[\mu_{J}, z_{J}^{*}\right]_{n} \mathfrak{a}_{n}^{*}\right)\right)
$$

and details can be found in the next section 3 (see especially section 3.5).

Clearly, this description of balanced networks is as asymmetric w.r.t. vertical/horizontal symmetry as only possible. Thus, it does not respect most of interesting dualities, which appear as non-trivial properties of the answers. Instead, it is extremely simple and very close to conventional matrix model techniques. In particular, it provides a very simple description of infinitesimal symmetries (Ward identities), and this is some compensation for non-transparency of large invariances (dualities). Moreover, as mentioned in the Introduction, the Ward identities are now labeled by sections of the network. The description is simple when the sections are pure vertical, but they can be easily deformed to include horizontal pieces, and the study of such cases can bring us closer to description of spectral dualities, even in this asymmetric formalism.

\subsection{Variation X: $q$-deformation}

The main new thing at this level is Jackson discretization of integrals:

$$
\int_{0}^{z} f(x) d x \longrightarrow(1-q) \sum_{n>0} z q^{n} f\left(q^{n} z\right)
$$

It can seem that there is a problem here, because the screening charges would require integrals along closed contours, and one may think the Jackson integral is not their good counterpart. What makes this deformation possible is the fact that the screening charges in the DF matrix models of [24-30] are actually defined along open contours between ramification points.

The most important result of discretization is the Young diagram expansion for exponentiated screening in fermionic realization (2.41) (an avatar of the Cauchy formula):

$$
\begin{aligned}
& \exp (\hat{Q})=\exp \left(\oint \psi_{a}^{+}(x) \psi_{a+1}^{-}(x) d x\right) \\
& \longrightarrow \exp \left((1-q) \sum_{n>0}^{\infty} q^{n} \psi_{a}^{+}\left(q^{n}\right) \psi_{a+1}^{-}\left(q^{n}\right)\right)=\sum_{\lambda} \frac{q^{|\lambda|}}{\zeta_{\lambda}} \Psi_{a, \lambda}^{+} \Psi_{a+1, \lambda}^{-}
\end{aligned}
$$

where $\lambda=\left\{\lambda_{1} \geq \lambda_{2} \geq \ldots \geq \lambda_{l(\lambda)}>0\right\}$ is the Young diagram with $|\lambda|=\sum_{i=1}^{l(\lambda)} \lambda_{i}$ boxes, $\Psi_{\lambda}=\prod_{i} \psi\left(q^{\lambda_{i}}\right)$ and $\zeta_{\lambda}=\prod_{r} m_{r}$ !, where $m_{r}$ is a number of times $r$ appears in the partition $\lambda$. This formula is a simple avatar of the Cauchy expansion. 
Operators of the type $\psi_{\lambda}$ play a crucial role in building particular network models: they are intertwiners of peculiar representations of DIM and their matrix elements are the topological vertices (perhaps, refined) within the topological string framework. Since elements of some Verma modules of double affine algebra $\operatorname{DIM}\left(\mathfrak{g l}_{1}\right)$ are labeled by $3 \mathrm{~d}$ Young diagrams (just like Verma modules of affine Virasoro by ordinary Young diagrams), topological vertices are naturally expressed as sums over plane partitions.

\subsection{Variation XI: $q, t, \ldots$-deformations}

Everything, what we surveyed above is straightforwardly deformed, at least from Schur to the Macdonald level, or, in group theory terms, from ordinary and affine (current) to double affine algebras DIM. Moreover, one can expect a topicality of the elliptic and further Kerov deformations, and, perhaps, even further, to triple-affine Pagoda algebras of [50], at least, to those corresponding to the double elliptic systems.

A short list of algebraic deformations is (in accordance with the columns: dimension|deformation parameters|symmetric polynomials|algebra of symmetry):

$$
\begin{aligned}
& 4 d t=1, q=1 \quad \text { Schur } \quad \text { Virasoro } / \mathcal{W}_{1+\infty} \\
& 4 d t=q^{\beta}, q \rightarrow 1 \quad \text { generalized Jack affine Yangian } \\
& 5 d \quad t=q \quad \text { Schur } \quad q-\text { Virasoro } \\
& 5 d \quad t, q \quad \text { generalized Macdonald DIM }
\end{aligned}
$$

From the gauge theory/string perspective, the deformation parameters are associated with compactification radius of the fifth dimension $R_{5}$ :

$$
q=e^{\epsilon_{1} R_{5}}, \quad t=e^{-\epsilon_{2} R_{5}}
$$

One naturally expects more parameters: the $q, t$ probably can be lifted to a three-parameter deformation associated with F-theory compactified on an elliptically fibred Calabi-Yau four-fold. Some evidence that the Seiberg-Witten/Nekrasov theory survives in a nice form beyond the Macdonald $q$, $t$-deformation is provided by the double elliptic studies on integrability side [155-159] and by reinterpretation of the Seiberg duality for $N_{f}>2 N_{c}$ [160-164] in terms of topological strings [165-169]. It remains to repeat once again that potential of the DIM algebras is also far from being exhausted by the $q, t$-deformation.

From CFT perspective, the most natural is the $\beta$-deformation, $t=q^{\beta}, \beta=\sqrt{\epsilon_{1} / \epsilon_{2}}$, which shifts the Virasoro central charge away from unity and other integer values in the multi-field case. As to the $q$-like deformations, they are long known to be natural for hypergeometric series and their generalizations, which CFT is really about. One of the main new things is that the stress tensor and more general $\mathcal{W}_{K}$ generators are now unified: they are all combinations of primary vertex operators, form a closed subalgebra and possess a nonvanishing centralizer so that one can consider models with the corresponding symmetry.

Another interesting point is a drastic increase of applicability domain for fermionization: after discretization of screening integrals, it continues to work in many representations beyond $c=1$, moreover, the fermionic intertwiners in DIM are actually the refined topological vertices from topological string theory. 
The most impressive result of deformation is clear unification of a huge variety of notions and phenomena, which appeared in different branches of science. It gets clear that they were describing the same things, just in different interpretations and limits, about one and the same object: the network matrix model, which is no more than a generic DIM-symmetric partition function on graphs.

In the last part of this section, we briefly consider the peculiarities of the simplest deformation, $t=q^{\beta}, q \rightarrow 1$.

\subsection{Variation XII: $\beta$-deformation to non-unit Virasoro central charge}

The main new thing at non-unit $\beta=\log t / \log q$ as compared with subsection 2.0 is that the Vandermonde determinants in the matrix model measure are raised to power $2 \beta$ instead one 2 , i.e. the matrix models are lifted to the $\beta$-ensembles [24-30, 104, 170-174], what leads to a temporal loss of connection to integrability theory (which is presumably restored after the $q$-deformation). Anyhow, technically most formulas are obtained by analytical continuation from integer values of $\beta$. The possibility to do so (unambiguously) comes from $\beta$ polynomiality of the Selberg integrals, which define most correlators in the DF $\beta$-ensembles.

In the conformal field theory representation [104], the $\beta$-ensemble corresponds to theory with non-unit central charge. As already mentioned, for non-integer Virasoro central charge $c$ one can expect problems with fermionization: only bosonization is straightforward. However, an appropriate substitute of fermionized formulas actually survives all the deformations, all the way to DIM, at least in some representations (not restricted to $\beta=1$ ).

Screening charge $\hat{Q}$, acting on the Fock space $\mathcal{F}_{\alpha}=\left\{\operatorname{Pols}\left(\tau_{n}\right)\right\} \cdot e^{\alpha T_{0}}$, is

$$
\begin{aligned}
\hat{Q} & =\oint \hat{S}(x) d x=\underbrace{\operatorname{res}_{x=0} \hat{S}(x)}_{\sum_{n} x^{n} \chi_{n}\{\tau\}} \\
\hat{S}(x) & =: e^{\sqrt{2 \beta} \phi(x)}:=e^{\exp \left(\sum_{n>0} \frac{\sqrt{\beta} \tau_{n} x^{n}}{n}\right)} e^{\sqrt{\beta} T_{0}} x^{2 \sqrt{\beta} \partial_{0}} \exp \left(-\sum_{n} \frac{\sqrt{\beta}}{n x^{n}} \frac{\partial}{\partial \tau_{n}}\right)
\end{aligned}
$$

where $\chi_{n}\{p\}$ are the characters of symmetric representations $[n]$ of $s l$ algebras (the Jack polynomials in this particular case). Applied to the highest-weight state

$$
|m+1\rangle=e^{-\alpha_{r, s} T_{0}}, \quad \alpha_{r, s}=(1+r) \frac{\sqrt{\beta}}{2}-(1+s) \frac{1}{2 \sqrt{\beta}}
$$

it gives

$$
\hat{Q}\left|\alpha_{-1, m}\right\rangle=\chi_{m}\{\tau\}\left|\alpha_{1, m}\right\rangle
$$

Similarly

$$
\hat{Q}^{r}\left|\alpha_{-r, m}\right\rangle=\chi_{\left[m^{r}\right]}\{\tau\}\left|\alpha_{r, m}\right\rangle
$$

These screening charge commutes

$$
\left[\hat{L}_{n}, \hat{Q}\right]=0
$$


with the Virasoro generators

$\hat{L}_{n}=\sum_{k}(k+n) \tau_{k} \frac{\partial}{\partial \tau_{k+n}}+\sum_{k=1}^{n-1} k(n-k) \frac{\partial^{2}}{\partial \tau_{k} \partial \tau_{n-k}}+2 n \sqrt{\beta} \frac{\partial^{2}}{\partial \tau_{n} \partial T_{0}}-n(n+1) \mathfrak{Q} \frac{\partial}{\partial \tau_{n}}, \quad n>0$

where $\mathfrak{Q}=\sqrt{\beta}-\frac{1}{\sqrt{\beta}}$. Then, one obtains

$$
\hat{L}_{n} \chi_{\left[m^{r}\right]}=2 n \sqrt{\beta} \alpha_{r, m} \frac{\partial \chi_{\left[m^{r}\right]}}{\partial \tau_{n}} \quad n>0
$$

while the action of

$$
\hat{L}_{0}=\sum_{k} k \tau_{k} \frac{\partial}{\partial \tau_{k}}+\beta \frac{\partial^{2}}{\partial T_{0} \partial T_{0}}+(1-\beta) \frac{\partial}{\partial T_{0}}
$$

still gives the size of the Young diagram:

$$
\hat{L}_{0} \chi_{\left[m^{r}\right]}=m r \cdot \chi_{\left[m^{r}\right]}
$$

The negative modes are:

$$
\hat{L}_{-n}=\sum_{k} k \tau_{k+n} \frac{\partial}{\partial \tau_{k}}+\sqrt{\beta} \tau_{n} \frac{\partial}{\partial T_{0}}+\frac{1}{4} \sum_{k=1}^{n-1} \tau_{k} \tau_{n-k}+\frac{n-1}{2} \mathfrak{Q} \tau_{n}
$$

so that

$$
\left[\hat{L}_{n}, \hat{L}_{m}\right]=(n-m) \hat{L}_{n+m}+\frac{n\left(n^{2}-1\right)}{12}\left(1-6 \mathfrak{Q}^{2}\right) \delta_{n+m, 0}
$$

and the current modes are now

$$
\hat{J}_{-n}=\frac{\tau_{n}}{\sqrt{2}}, \quad \hat{J}_{0}=\sqrt{2 \beta} \frac{\partial}{\partial T_{0}}, \quad \hat{J}_{n}=\sqrt{2} n \frac{\partial}{\partial \tau_{n}}
$$

while the Sugawara relation is

$$
\hat{T}(z)=: \frac{1}{2} \hat{J}(z)^{2}:+\frac{\mathfrak{Q}}{\sqrt{2}} \partial_{z} J(z)
$$

In terms of generating functions, the Ward identity (2.59), i.e. the corollary of symmetry (2.57) becomes

$$
\left[z^{2} \hat{T}(z)-\sqrt{2 \beta} \alpha_{r, m} z \hat{J}(z)\right]_{-} \cdot \chi_{\left[m^{r}\right]}=0
$$

or

$$
\left(z^{2} \hat{T}(z)-\sqrt{2 \beta} \alpha_{r, m} z \hat{J}(z)\right) \cdot \chi_{\left[m^{r}\right]}=\operatorname{Pol}(z)
$$

Now similarly to obtaining (2.28), we can get the matrix element that is given by the integral ( $\beta$-ensemble) representation. It looks like

$$
\begin{aligned}
\chi_{\left[m^{r}\right]}\{\tau\} & =\left\langle\alpha_{r, m}\left|\hat{C}\left\{\tau_{n} / \sqrt{2}\right\} \hat{\mathcal{Q}}^{r}\right| \alpha_{-r, m}\right\rangle \\
& =-\frac{1}{r !} \underbrace{\oint \ldots \oint}_{r}\left(\prod_{i=1}^{r} \frac{\mathcal{G}\left\{\tau \mid x_{i}\right\} d x_{i}}{x_{i}^{m+r}}\right) \prod_{i<j}\left(x_{i}-x_{j}\right)^{2 \beta}=\langle 1\rangle_{\mathrm{DF}_{\mathrm{m}, \mathrm{r}}}
\end{aligned}
$$

However, the symmetric function $\chi_{\left[m^{r}\right]}\{\tau\}$ is now not the Schur, but the Jack polynomial. 
The Ward identity (2.29) is now substituted by

$$
\begin{aligned}
& \left(z^{2} \hat{T}(z)-\sqrt{2 \beta} \alpha_{r, m} z \hat{J}(z)\right)\langle 1\rangle_{\mathrm{DF}_{\mathrm{m}, \mathrm{r}}}=\operatorname{Pol}(z) \\
& =\left\langle\sum_{k, i} \frac{\tau_{k} x_{i}^{k+1}}{z-x_{i}}+\sum_{i, j=1}^{r} \frac{x_{i} x_{j}}{\left(z-x_{i}\right)\left(z-x_{j}\right)}-\mathfrak{Q} \sum_{i} \frac{z^{2}}{\left(z-x_{i}\right)^{2}}-2 \sqrt{\beta} \alpha_{r, m} \sum_{i} \frac{x_{i}}{\left(z-x_{i}\right)}\right\rangle_{\mathrm{DF}_{\mathrm{m}, \mathrm{r}}}
\end{aligned}
$$

One has to get two important points from considering this $\beta$-deformation:

- The deformation preserves the structure of equations and the vertex operators, moderately changing only the screening charges (the change that can be removed to a rescaling of the Heisenberg algebra operators), while the main change is due to changing the Sugawara relation, i.e. the construction of the Virasoro/W algebra.

- Matrix models partition functions are also changed moderately, basically with only the Vandermonde determinant being deformed (hence, changing the Ward identities).

These two properties will persist in the generic $q, t$-case, as we demonstrate in the next sections.

In fact, one could repeat this matrix model consideration in the deformed case with non-unit $q$, following the lines of [175-181]. However, the actual symmetry in this case becomes much larger than the Virasoro algebra: it is the DIM algebra, and we start its general description in the next section.

\section{DIM calculus for balanced network model}

In this section, we demonstrate how to deal with the balanced network model by methods of the DIM algebra, which is a development based on the previous consideration in [31$34,48,50,182]$. It is rather special from the algebraic perspective: only the $\operatorname{DIM}\left(g l_{1}\right)$ algebra with special values of central charges and rather peculiar representations allowing straightforward bosonization and even fermionization is considered, however, this covers almost all what is presently known about Nekrasov partition functions.

Details on various DIM algebras and their simplest representations are provided in the appendix, which can be useful for further development of the theory.

\subsection{DIM algebra}

Let us first remind the definition of the DIM algebra $U_{q, t}\left(\widehat{\widehat{\mathfrak{g l}}}_{1}\right)$. It looks like a deformation of the affine quantum algebra $U_{q}\left(\widehat{\mathfrak{g l}}_{2}\right)$ with the four Drinfeld currents: the positive/negative root generators $x^{ \pm}(z)=\sum_{n \in \mathbb{Z}} x_{n}^{ \pm} z^{-n}$, two exponentiated Cartan generators $\psi^{+}(z)$ and $\psi^{-}(z)$, which are power series in $z^{-1}$ and $z$ correspondingly, and the central element $\gamma$.

Commutation relations are

$$
\begin{aligned}
G^{\mp}(z / w) x^{ \pm}(z) x^{ \pm}(w) & =G^{ \pm}(z / w) x^{ \pm}(w) x^{ \pm}(z) \\
{\left[x^{+}(z), x^{-}(w)\right] } & =\frac{(1-q)\left(1-t^{-1}\right)}{1-q / t}\left(\delta\left(\gamma^{-1} z / w\right) \psi^{+}\left(\gamma^{1 / 2} w\right)-\delta(\gamma z / w) \psi^{-}\left(\gamma^{-1 / 2} w\right)\right)
\end{aligned}
$$




$$
\begin{aligned}
\psi^{ \pm}(z) \psi^{ \pm}(w) & =\psi^{ \pm}(w) \psi^{ \pm}(z) \\
\psi^{+}(z) \psi^{-}(w) & =\frac{g(\gamma w / z)}{g\left(\gamma^{-1} w / z\right)} \psi^{-}(w) \psi^{+}(z) \\
\psi^{+}(z) x^{ \pm}(w) & =g\left(\gamma^{\mp 1 / 2} w / z\right)^{\mp 1} x^{ \pm}(w) \psi^{+}(z) \\
\psi^{-}(z) x^{ \pm}(w) & =g\left(\gamma^{\mp 1 / 2} z / w\right)^{ \pm 1} x^{ \pm}(w) \psi^{-}(z) \\
\operatorname{Sym}_{z_{1}, z_{2}, z_{3}} z_{2} z_{3}^{-1} & {\left[x^{ \pm}\left(z_{1}\right),\left[x^{ \pm}\left(z_{2}\right), x^{ \pm}\left(z_{3}\right)\right]\right]=0 }
\end{aligned}
$$

The DIM algebra is a Hopf algebra with comultiplication

$$
\begin{aligned}
& \Delta\left(\psi^{ \pm}(z)\right)=\psi^{ \pm}\left(\gamma_{2}^{ \pm 1 / 2} z\right) \otimes \psi^{ \pm}\left(\gamma_{1}^{\mp 1 / 2} z\right) \\
& \Delta\left(x^{+}(z)\right)=\psi^{-}\left(\gamma_{1}^{1 / 2} z\right) \otimes x^{+}\left(\gamma_{1} z\right)+x^{+}(z) \otimes 1 \\
& \Delta\left(x^{-}(z)\right)=1 \otimes x^{-}(z)+x^{-}\left(\gamma_{2} z\right) \otimes \psi^{+}\left(\gamma_{2}^{1 / 2} z\right)
\end{aligned}
$$

where $\gamma_{1}^{ \pm 1 / 2}=\gamma^{ \pm 1 / 2} \otimes 1, \quad \gamma_{2}^{ \pm 1 / 2}=1 \otimes \gamma^{ \pm 1 / 2}$ and the functions $g(z)=\frac{G^{+}(z)}{G^{-}(z)}$ is restricted by the associativity requirement $g(z)^{-1}=g\left(z^{-1}\right)$. We omit expression for the counit and antipode, since we will not need them.

This data allows one to construct the universal $R$-matrix [67-69].

In these relations, $\gamma^{ \pm 1 / 2}$ and $\psi_{0}^{+} \equiv \psi^{+}(z=\infty), \psi_{0}^{-} \equiv \psi^{-}(z=0)$ are the central elements. Parameterizing their values as

$$
\gamma=\omega^{-M_{1}}, \quad \psi_{0}^{ \pm}=\omega^{ \pm M_{2}}, \quad \omega \equiv \sqrt{\frac{q}{t}}
$$

we reproduce the $\left(M_{1}, M_{2}\right)$ pairs of integers enumerating representations in section 2.9. The action of this comultiplication increases the central charges, in contrast with the MooreSeiberg comultiplication $\Delta_{M S}(2.45)$. This is why the number of free fields is also increased by action of the comultiplication. In particular, starting from one free field (Kac-Moody level), we produce the Virasoro by acting with comultiplication, which adds yet another free field etc. Of the two integers $M_{1}$ and $M_{2}$, the first one is a counterpart of the Kac-Moody algebra level so that the refined topological vertex is a matrix element of the operator intertwining the level one representations, i.e. it can be realized by one free field. We explain this construction manifestly in the next subsections.

The structure of the algebra is encoded in the function $G(z)$ which is often chosen to be cubic in $z$ with additional restriction $q_{1} q_{2} q_{3}=1$ :

$$
G^{ \pm}(z)=\left(1-q_{1} z\right)\left(1-q_{2} z\right)\left(1-q_{3} z\right)=\left(1-q^{ \pm 1} z\right)\left(1-t^{\mp 1} z\right)\left(1-(q / t)^{\mp 1} z\right)
$$

Without any harm to commutation relations and comultiplication, it can be further promoted to unrestricted $q_{1,2,3}$ and more general Kerov deformations, and even to the elliptic function, though details of bosonization procedure below should still be worked out in these cases. 


\subsection{Bosonization in the case of special slopes}

Explicit expressions for $C_{I J K}$ are currently known only for particular slopes: $\vec{s}^{\prime \prime}=(0,1)$ and $\vec{s}^{\prime}=(1, M), \vec{s}^{\prime \prime \prime}=(1, M \pm 1)$, see figure 5 . According to [66], they can be expressed in terms of the following bosonization:

$$
\begin{aligned}
\phi(z) & =\sum_{n>0}\left(\frac{1-t^{n}}{1-q^{n}} \frac{z^{n} a_{-n}}{n}-\frac{1-t^{n}}{1-q^{n}} \frac{a_{n}}{n z^{n}}\right), \\
{\left[a_{n}, a_{m}\right] } & =n \frac{1-q^{|n|}}{1-t^{|n|}} \delta_{m+n, 0}
\end{aligned}
$$

From this free field we can construct pre-vertex operators depending on infinitely many time-variables $p_{n}$ :

$$
\begin{aligned}
C\{p\} & =\exp \left(\sum_{n>0} \frac{1-t^{n}}{1-q^{n}} \frac{a_{n}}{n} p_{n}\right) \\
\bar{C}\{p\} & =\exp \left(-\sum_{n>0} \frac{1-t^{n}}{1-q^{n}} \frac{a_{n}}{n} p_{n}\right) \\
C^{\dagger}\{p\} & =\exp \left(\sum_{n>0} \frac{1-t^{n}}{1-q^{n}} \frac{a_{-n}}{n} p_{n}\right) \\
\bar{C}^{\dagger}\{p\} & =\exp \left(-\sum_{n>0} \frac{1-t^{n}}{1-q^{n}} \frac{a_{-n}}{n} p_{n}\right)
\end{aligned}
$$

with

$$
C^{\dagger}\left\{p_{n}=\sum_{i} z_{i}^{n}\right\} \bar{C}\left\{p_{n}=\sum_{i} z_{i}^{-n}\right\}=: \prod_{i} e^{\phi\left(z_{i}\right)}:
$$

These operators can be used to define the main vertex operators for the above-mentioned particular slopes:

$$
\begin{aligned}
& \Psi_{\lambda}(z)=\Psi\left[\underset{-u z}{M} \cdot{ }_{-M}^{z, \lambda}-1\right]=\frac{(-z)^{M|\lambda|}}{c_{\lambda}\left(f_{\lambda}\right)^{M}} C^{\dagger}\left[t^{-1} q^{\lambda} t^{\rho+1 / 2} z\right] \bar{C}\left[q^{-\lambda} t^{-\rho-1 / 2} q z^{-1}\right] \times(-u z)^{|\lambda|} q^{n\left(\lambda^{T}\right)}
\end{aligned}
$$

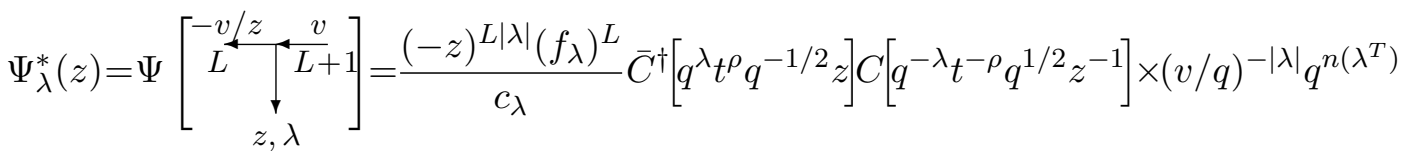

Here only one Young diagram $\lambda$ is shown explicitly, the two others label matrix elements of the operator. The operator acts on the Fock space $\mathcal{F}_{u}$, in which the basis vectors are labelled by Young diagrams (e.g. the Schur functions provide a basis, $\chi_{Y}\left(a_{-n}\right)|u, \varnothing\rangle$ ). The edge parameters/lengths $Q$ are encoded in the spectral parameters $u$ and $z$. More precisely, edge lengths are given by ratios of the spectral parameters between the parallel lines, as shown in figure 5 , a). Notice that the vertices $\Psi, \Psi^{*}$ depend only on two spectral parameters, the third one being determined by the momentum conservation condition. 
This condition follows from the requirement that the vertices intertwine the action of the zero mode of the generator $x^{+}(z)$. An additional notation is:

$f_{\lambda}=\prod_{(i, j) \in \lambda}(-) \cdot q^{j-1 / 2} t^{1 / 2-i}, \quad c_{\lambda}=\prod_{(i, j) \in \lambda}\left(1-q^{\lambda_{i}-j} t^{\lambda_{j}^{T}-i+1}\right), \quad n\left(\lambda^{T}\right)=\sum_{(i, j) \in \lambda}(j-1)$

where $\lambda_{j}^{T}$ are row lengths of transposed Young diagram. Finally,

$$
C\left[q^{\lambda} t^{\rho+1 / 2}\right]=C\left\{p_{n}=\sum_{i \geq 1}\left(q^{n \lambda_{i}}-1\right) t^{n(1-i)}+\frac{1}{1-t^{-n}}\right\} \stackrel{|t|>1}{=} C\left\{p_{n}=\sum_{i \geq 1} q^{n \lambda_{i}} t^{n(1-i)}\right\}
$$

Here the requirement $|t|>1$ is needed for convergence of the sum. However, the result is analytic, and thus valid for any complex $t \neq 1$.

The Feynman diagram is made from horizontal lines and vertical segments between them. Operators along the horizontal lines are simply multiplied, but each horizontal line depends on its own free field, i.e. with $K$-line diagram we associate operators acting in the $K$-th tensor power of the single field Fock space, $\mathcal{F}_{u_{1}} \otimes \cdots \otimes \mathcal{F}_{u_{K}}$. Sum over the Young diagrams, $\lambda$ on vertical segments is performed with the simple weight, which is independent of the edge length $Q$ : all $Q$-dependent factors are already included in the definitions of $\Psi$. One can understand this procedure as cutting the propagators $\Pi^{I J}$ in two halves (taking a "square root") and attaching the resulting stubs to the corresponding adjacent vertices.

\subsection{Relation to topological vertex}

The operator $C\{p\}$ defined in eq. (3.6) switches between the Fock space and the time variables: for the vacuum state annihilated by all operators $a_{-n}$ with $n>0,\langle 0| a_{-n}=0$ we have

$$
\begin{aligned}
\langle 0| C\{p\} a_{-n} & =p_{n}\langle 0| C\{p\} \\
\langle 0| C\{p\} a_{n} & =n \frac{1-q^{n}}{1-t^{n}} \frac{\partial}{\partial p_{n}}\langle 0| C\{p\}
\end{aligned}
$$

This $\langle 0| C\{p\}$ is a $p$-dependent set of common coherent eigenstates of all the annihilation operators $a_{-n}$. Accordingly, one can use the Macdonald polynomials $M_{\lambda}\{p\}$ to define "Macdonald states":

and their involutions

$$
M_{\lambda}\{p\}=\left\langle 0|C\{p\}| M_{\lambda}\right\rangle
$$

$$
\bar{M}_{\lambda}\{p\}=M_{\lambda}\{-p\}=\left\langle 0|\bar{C}\{p\}| M_{\lambda}\right\rangle
$$

The skew characters are given by the matrix elements

$$
M_{\lambda / \mu}\{p\}=\frac{\left\langle M_{\mu}|C\{p\}| M_{\lambda}\right\rangle}{\left\langle M_{\mu} \mid M_{\mu}\right\rangle}
$$

The matrix elements of the intertwiners $\Psi, \Psi^{*}$ in the basis of the Macdonald states give the standard expression for the AK version of the refined topological vertex $[36,37]$

$$
\left\langle\bar{M}_{\mu}|\Psi[\stackrel{z, \lambda}{\longleftarrow}]| \bar{M}_{\nu}\right\rangle=\left\|M_{\lambda}\right\|^{2}|| M_{\nu} \|^{2}\left(-\frac{t^{1 / 2} u}{q(-z)^{M}}\right)^{|\lambda|} f_{\lambda}^{-M}\left(t^{-1 / 2} z\right)^{|\mu|-|\nu|} f_{\nu}^{-1} C_{\nu}^{\mu \lambda}(q, t)
$$




$$
\left\langle\bar{M}_{\nu}|\Psi[\underset{z, \lambda}{\longleftarrow}]| \bar{M}_{\mu}\right\rangle=\left.|| M_{\mu}\right|^{2}\left(-\frac{q(-u)^{L}}{t^{1 / 2} z}\right) f_{\lambda}^{L}\left(t^{-1 / 2} u\right)^{-|\mu|+|\nu|} f_{\nu} C_{\mu \lambda}{ }^{\nu}(q, t)
$$

where

$$
\begin{aligned}
& C_{\mu \lambda}{ }^{\nu}(q, t)=M_{\lambda}^{(q, t)}\left[t^{\rho}\right] \sum_{\sigma} \bar{M}_{\mu^{T} / \sigma^{T}}^{(t, q)}\left[t^{-\lambda^{T}} q^{\rho}\right] M_{\nu / \sigma}^{(q, t)}\left[q^{\lambda} t^{\rho}\right](q / t)^{\frac{|\sigma|-|\nu|}{2}} f_{\nu}^{-1}(q, t), \\
& C_{\nu}^{\mu \lambda}(q, t)=(-1)^{|\lambda|+|\mu|+|\nu|} C_{\mu^{T} \lambda^{T}} \nu^{T}(t, q)
\end{aligned}
$$

The IKV vertices [35] arise in another basis: for the $q, t$-independent Schur states $\left|s_{\lambda}\right\rangle$ and their $q, t$-dependent duals $\left\langle S_{\lambda}\right|$ w.r.t. to the Macdonald scalar product.

\subsection{Building screening charges and vertex operators}

The screening charges and vertex operators of the Virasoro or $W_{K}$-algebra arise as combinations of intertwiners $\Psi, \Psi^{*}$. The screening charges should commute with the Virasoro generators, and since the Virasoro algebra is generated by an element of DIM algebra, the intertwiners of DIM are the natural candidates for the screening charges. We will see in the next section that one can interpret the commutation graphically. The Virasoro generators act on the horizontal lines, and the screening charges are segments of the vertical lines between the horizontal ones. There are also external vertical lines, which correspond to the Virasoro vertex operators. These do not commute with the Virasoro algebra, because the corresponding intertwiner contains an extra representation, the vertical one. The action of energy-momentum tensor on this additional representation gives extra terms, making the commutation rules nontrivial.

Screenings charges. Let us start by building the screening charges. They correspond to internal vertical lines in the web. The minimal example contains two intertwiners, which are contracted with each other to form a vertical segment between the adjacent horizontal lines. The whole procedure resembles the free fermion construction of the screening currents from section 2.6. Each intertwiner plays the role of a free fermion, so that their contraction gives rise to fermion bilinears, i.e. the screening currents of dimension one. The integral of the currents is replaced by the sum over intermediate states in the vertical representation as in section 2.10 .

The product of intertwiners is given by

$$
\begin{aligned}
& \stackrel{(1, M)}{\left(\Psi^{*}(1, M+1)\right.}
\end{aligned}
$$

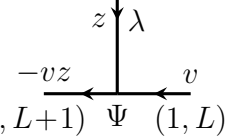

$$
\begin{aligned}
& =\sum_{\lambda}\left\|M_{\lambda}\right\|^{-2} \Psi_{\lambda}^{*}(z) \otimes \Psi_{\lambda}(z)= \\
& =\sum_{\lambda}\left(\frac{q v}{u}(-z)^{M-L+1}\right)^{|\lambda|} \frac{f_{\lambda}^{M-L-1} q^{2 n\left(\lambda^{T}\right)}}{c_{\lambda} c_{\lambda}^{\prime}} \exp \left\{-\sum_{n \geq 1} \frac{1}{n} \frac{1-t^{n}}{1-q^{n}}\left(1+\left(\frac{q}{t}\right)^{n}\right) p_{n}\left(q^{\lambda} t^{\rho} q^{-1 / 2} z\right) \tilde{\alpha}_{-n}\right\} \times \\
& \times \exp \left\{\sum_{n \geq 1} \frac{1}{n} \frac{1-t^{n}}{1-q^{n}}\left(1+\left(\frac{q}{t}\right)^{n}\right) p_{n}\left(q^{-\lambda} t^{-\rho} q^{1 / 2} z^{-1}\right) \tilde{\alpha}_{n}\right\}=
\end{aligned}
$$




$$
=\sum_{\lambda}\left(\frac{q v}{u}(-z)^{M-L+1}\right)^{|\lambda|} \frac{f_{\lambda}^{M-L-1} q^{2 n\left(\lambda^{T}\right)}}{c_{\lambda} c_{\lambda}^{\prime}}: \prod_{i \geq 1} S\left(q^{\lambda_{i}} t^{\rho_{i}} q^{-1 / 2} z\right):
$$

where $c_{\lambda}^{\prime}=\prod_{(i, j) \in \lambda}\left(1-q^{\lambda_{i}-j+1} t^{\lambda_{j}^{T}-i}\right)$ and

$S(x)=\exp \left\{-\sum_{n \geq 1} \frac{1}{n} \frac{1-t^{n}}{1-q^{n}}\left(1+\left(\frac{q}{t}\right)^{n}\right) x^{n} \tilde{\alpha}_{-n}\right\} \exp \left\{\sum_{n \geq 1} \frac{1}{n} \frac{1-t^{n}}{1-q^{n}}\left(1+\left(\frac{q}{t}\right)^{n}\right) x^{-n} \tilde{\alpha}_{n}\right\}$

We see that the contraction of two intertwiners depends on a particular ("Virasoro") combination of the bosonic oscillators acting on the two horizontal Fock representations: ${ }^{4}$

$$
\tilde{\alpha}_{n}=\frac{1}{1+\omega^{2|n|}}\left(a_{n}^{(1)}-\omega^{|n|} a_{n}^{(2)}\right), \quad \omega \equiv \sqrt{\frac{q}{t}}
$$

where $a_{n}^{(1)}=a_{n} \otimes 1$ and $a_{n}^{(2)}=1 \otimes a_{n}$. The generators $\tilde{\alpha}_{n}$ are normalized differently from the original Heisenberg generators $a_{n}$ (cf. eq. (3.5)):

$$
\left[\tilde{\alpha}_{n}, \tilde{\alpha}_{m}\right]=n \frac{1-q^{|n|}}{\left(1-t^{|n|}\right)\left(1+\omega^{2|n|}\right)} \delta_{n+m, 0}
$$

The contraction of intertwiners provides us with an indefinite number of screening currents, since the product in the last line of eq. (3.17) is infinite. This corresponds to the exponential of the screening charge and fits well with the picture where the pair of intertwiners gives fermion bilinear screening current:

$$
\sum_{\lambda}\left\|M_{\lambda}\right\|^{-2} \Psi_{\lambda}^{*}(z) \otimes \Psi_{\lambda}(z) \sim \exp (\oint S(x) d x)=\sum_{N} \frac{1}{N !} \oint \prod_{i=1}^{N} S\left(x_{i}\right) d^{N} x
$$

According to the $q$-deformation prescription from section 2.10, the positions of the screening currents are discrete and parameterized by the Young diagrams $\lambda$ :

$$
x_{i}=q^{\lambda_{i}} t^{\rho_{i}} q^{-1 / 2} z
$$

so that the contour integral in eq. (3.21) is replaced by the sum over $\lambda .{ }^{5}$ To get a definite number of screenings one should put some vertex operators and external states into the system. Then, the selection rules automatically provide one with a necessary number of screening charges. We will see this effect below, when discussing the vertex operators.

The operator product expansion of two screening operators immediately defines the corresponding matrix model measure. We have

$$
S(x) S(y) \sim \frac{\left(\frac{x}{y} ; q\right)_{\infty}\left(\frac{y}{x} ; q\right)_{\infty}}{\left(t \frac{x}{y} ; q\right)_{\infty}\left(t \frac{y}{x} ; q\right)_{\infty}}: S(x) S(y):
$$

\footnotetext{
${ }^{4}$ We conform with the notations of [50].

${ }^{5}$ One can understand this recipe in different ways: either as the Jackson integral, or as a sum over residues of the normal ordered operator expression. The final result for the sum over Young diagrams is the same in the both approaches.
} 
where $(x ; q)_{\infty}=\prod_{k \geq 1}\left(1-q^{k} x\right)$. This means that the matrix model is of $(q, t)$-deformed type, with the measure given by

$$
\Delta^{(q, t)}(x)=\prod_{i \neq j} \frac{\left(\frac{x_{i}}{x_{j}} ; q\right)_{\infty}}{\left(t \frac{x_{i}}{x_{j}} ; q\right)_{\infty}}
$$

It is known [31, 32, 80-82] that such a matrix model explicitly computes the Nekrasov partition function and the $\mathrm{Vir}_{q, t}$ conformal block.

Of course, the expression for the intermediate vertical segment between any two adjacent horizontal lines (e.g. $i$-th and $(i+1)$-th) has the same form as eq. (3.17). The only difference is that the Heisenberg generators are $a_{n}^{(i)}$ and $a_{n}^{(i+1)}$ instead of $a_{n}^{(1)}$ and $a_{n}^{(2)}$. On the tensor product of $K \geq 3$ Fock representations acts the $W_{K}$-algebra and the intermediate segments correspond to $(K-1)$ different screening charges commuting with this algebra. The combinations of the differences between the adjacent bosonic oscillators correspond to the roots of the $A_{K-1}$ algebra.

Vertex operators. As we have already mentioned, vertex operators should be built from the intertwiners with external vertical legs. Again, a minimal example contains a pair of intertwiners on two horizontal lines, which are, however, not contracted in this case. Their product now essentially depends on the both horizontal oscillators. This corresponds to a composite vertex operator having two parts: the Virasoro part depending on $\tilde{a}_{n}$ and the Heisenberg part depending on the orthogonal linear combination of the oscillators, $\bar{a}_{n}$. This is exactly as prescribed by the AGT relation [175-181, 183-185], where the Nekrasov functions for the gauge group $\mathrm{U}(N)$ correspond to the conformal block of the algebra $\operatorname{Vir}_{q, t} \otimes$ Heis $_{q, t}$.

We have the following result:

$$
\begin{aligned}
& \underset{-u w_{1} \Psi \leftarrow u}{\left.\stackrel{w_{1}}{\longleftarrow}\right\rfloor^{\varnothing}(1, M)} \\
& =\Psi_{\varnothing}\left(w_{1}, v\right) \otimes \Psi_{\varnothing}^{*}\left(w_{2}, u\right)=\widetilde{V}_{w_{1} / w_{2}}^{\mathrm{Vir}}\left(\left(w_{1} w_{2}\right)^{1 / 2}\right) V_{w_{1} / w_{2}}^{\mathrm{Heis}}\left(\left(w_{1} w_{2}\right)^{1 / 2}\right) \\
& \frac{-v / w_{2} \Psi^{*}}{\left.\left.(1, L) \oiiint_{w_{2}}^{\longleftarrow}\right|_{\varnothing} ^{(1, L}+1\right)}
\end{aligned}
$$

where the indices denote the Liouville-like momenta of the vertex operators

$$
\begin{aligned}
\widetilde{V}_{P}^{\mathrm{Vir}}(z)= & \exp \left\{-\sum_{n \geq 1} \frac{P^{n / 2}+P^{-n / 2}}{n\left(1-q^{n}\right)} z^{n} \tilde{\alpha}_{-n}\right\} \exp \left\{-\sum_{n \geq 1} \frac{q^{n}\left(P^{n / 2}+P^{-n / 2}\right)}{n\left(1-q^{n}\right)} z^{-n} \tilde{\alpha}_{n}\right\}, \\
V_{P}^{\mathrm{Heis}}(z)= & \exp \left\{-\sum_{n \geq 1} \frac{\omega^{-n}\left(\left(\omega^{2} P\right)^{n / 2}-\left(\omega^{2} P\right)^{-n / 2}\right)}{n\left(1-q^{n}\right)} z^{n} \bar{\alpha}_{-n}\right\} \\
& \times \exp \left\{-\sum_{n \geq 1} \frac{(q t)^{n / 2}\left(\left(P / \omega^{2}\right)^{-n / 2}-\left(P / \omega^{2}\right)^{n / 2}\right)}{n\left(1-q^{n}\right)} z^{-n} \bar{\alpha}_{n}\right\} .
\end{aligned}
$$


where $\tilde{\alpha}_{n}$ are defined in eq. (3.19) and

$$
\bar{\alpha}_{n}=\frac{\omega^{|n|}}{1+\omega^{2|n|}}\left(\omega^{|n|} a_{n}^{(1)}+a_{n}^{(2)}\right)
$$

Notice that the momenta in the U(1) part are slightly different (by $t^{2} / q^{2}$ ) for the positive and negative modes which matches the AGT prescription [41, 186-188].

The vertex operator (3.26), though it depends on the right combination of the oscillators $\tilde{\alpha}_{n}$ is not the full Virasoro vertex operator (in particular, it does not have a smooth limit for $t, q \rightarrow 1)$. The same comment actually applies to the exponential of the screening charge (3.17). The reason for this behavior is that both (3.17) and (3.25) are not balanced. This means that either incoming or outgoing representations are not horizontal. To get the balanced combination, one should consider the product of (3.17) and (3.25), i.e. the partial contraction of four intertwiners:

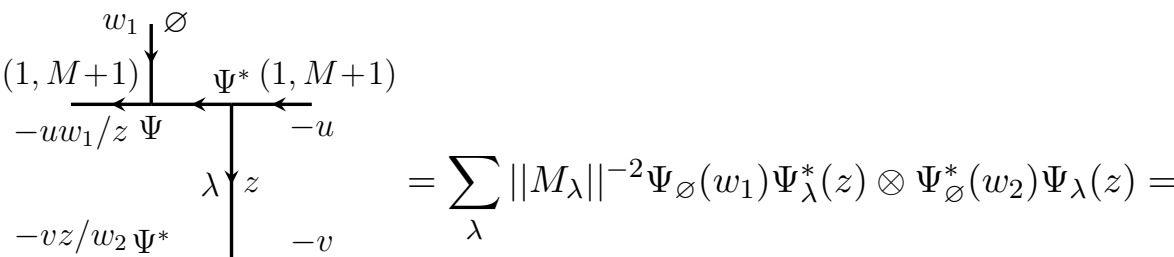

$$
\begin{aligned}
& (1, L) \\
& \begin{array}{l}
\qquad \prod_{w_{2}} \begin{array}{r}
\Psi(1, L) \\
\end{array} \\
\quad=V_{w_{1} / w_{2}}^{\mathrm{Heis}}\left(\left(w_{1} w_{2}\right)^{1 / 2}\right) \oint \prod_{i=1}^{N} S\left(x_{i}\right) d^{N} x V_{\left(t w_{2}\right) /\left(q w_{1}\right)}^{\mathrm{Vir}}\left(\left(w_{1} w_{2} q / t\right)^{1 / 2}\right),
\end{array}
\end{aligned}
$$

where $t^{N}=\sqrt{\frac{t}{q}} \frac{z}{w_{1}}$ and

$$
\begin{aligned}
V_{P}^{\mathrm{Vir}}(x)= & \exp \left(-\sum_{n \geq 1} \frac{1}{n} \frac{x^{n}}{1-q^{n}} \tilde{\alpha}_{-n}\left(P^{n / 2}-P^{-n / 2}\right)\right) \\
& \times \exp \left(-\sum_{n \geq 1} \frac{1}{n} \frac{q^{n}}{1-q^{n}} x^{-n} \tilde{\alpha}_{n}\left(P^{-n / 2}-P^{n / 2}\right)\right)
\end{aligned}
$$

Of course, one can change $w_{1}$ to $w_{2}$ and vice versa in all the formulas. The balanced combination of the operators automatically fixes two problems: it determines the number of screening charges $N$ and gives the correct expression for the $\operatorname{Vir}_{q, t}$ vertex operator (3.30) in terms of free fields $[175,176]$.

Other combinations of four intertwiners. In this paragraph we give an exhaustive list of webs, both balanced and unbalanced, obtained from combinations of four intertwiners on two horizontal lines. The first possibility is given by eq. (3.29), the second we describe below in (3.42), when we discuss conformal blocks. Here we consider two more variations. 


\section{Two antiparallel lines.}

$$
\begin{aligned}
& (1, M+1)
\end{aligned}
$$

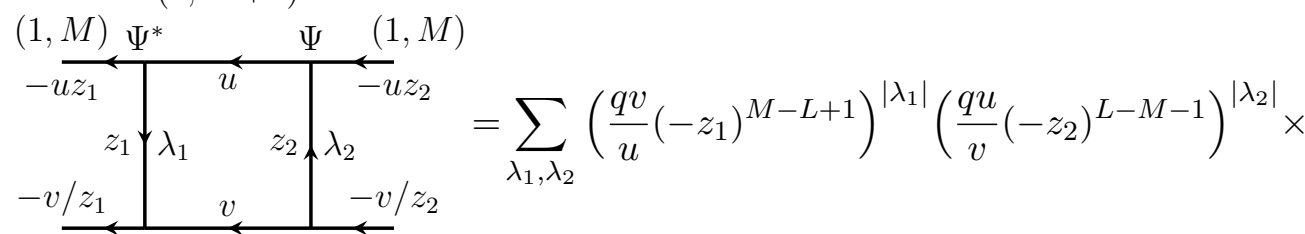

$$
\begin{aligned}
& (1, L+1) \Psi \quad(1, L) \Psi^{*}(1, L+1) \\
& \times \frac{f_{\lambda_{1}}^{M-L-1} f_{\lambda_{2}}^{M-L-1} q^{2 n\left(\lambda_{1}^{T}\right)+2 n\left(\lambda_{2}^{T}\right)}}{c_{\lambda_{1}} c_{\lambda_{1}}^{\prime} c_{\lambda_{2}} c_{\lambda_{2}}^{\prime}}: \prod_{i \geq 1} S_{2}\left(q^{\lambda_{2, i}} t^{\rho_{i}} q^{-1 / 2} z_{2}\right):: \prod_{j \geq 1} S_{1}\left(q^{\lambda_{1, j}} t^{\rho_{j}} q^{-1 / 2} z_{1}\right):
\end{aligned}
$$

Notice that here $S_{2}$ depends on the combination of the oscillators corresponding to the affine (imaginary) root of the algebra $\widehat{A}_{1}$ :

$$
\begin{array}{llrl}
\tilde{\alpha}_{n}^{(2)} & =\frac{1}{1+\omega^{2 n}}\left(a_{n}^{(2)}-\omega^{n} a_{n}^{(1)}\right), & & n \geq 1 \\
\tilde{\alpha}_{-n}^{(2)} & =\frac{1}{1+\omega^{2 n}}\left(a_{-n}^{(2)}-\omega^{n} a_{-n}^{(1)}\right), & & n \geq 1
\end{array}
$$

This diagram is balanced and corresponds to a particular case of the compactified toric diagram. The two antiparallel vertical lines should be understood as living on the two sides of the cylinder. We will give a more general "quasi-periodic" version of this diagram in section 3.7 , where we describe the affine $(q, t)$-matrix model.

2. Horizontal cut. This strange variation is obtained by adding two "internal" lines ending at empty diagrams:

$$
\begin{aligned}
& (1, M) \underset{\Psi^{*}}{(1, M+1)} \Psi^{*}(1, M+2)
\end{aligned}
$$

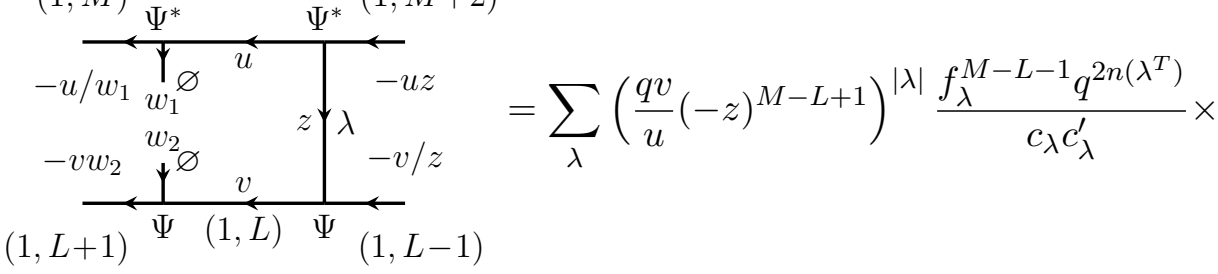

$$
\begin{aligned}
& \times \widehat{V}_{w_{1} / w_{2}}^{\mathrm{Heis}}\left(\sqrt{w_{1} w_{2}}\right) \widehat{V}_{w_{1} / w_{2}}^{\mathrm{Vir}}\left(\sqrt{w_{1} w_{2}}\right): \prod_{j=1}^{\tilde{N}} S\left(q^{\lambda_{j}} t^{\rho_{j}} q^{-1 / 2} z\right):
\end{aligned}
$$

where $t^{\tilde{N}}=\frac{z}{w_{1}}$ and

$$
\begin{aligned}
\widehat{V}_{P}^{\mathrm{Vir}}(z)= & \exp \left\{-\sum_{n \geq 1} \frac{\omega^{n}\left(P^{n / 2}-P^{-n / 2}\right)}{n\left(1-q^{n}\right)} z^{n} \tilde{\alpha}_{-n}\right\} \\
& \times \exp \left\{-\sum_{n \geq 1} \frac{q^{n} \omega^{n}\left(P^{-n / 2}-P^{n / 2}\right)}{n\left(1-q^{n}\right)} z^{-n} \tilde{\alpha}_{n}\right\},
\end{aligned}
$$




$$
\begin{aligned}
\widehat{V}_{P}^{\text {Heis }}(z)= & \exp \left\{-\sum_{n \geq 1} \frac{\omega^{-n}\left(P^{n / 2}-P^{-n / 2}\right)}{n\left(1-q^{n}\right)} z^{n} \bar{\alpha}_{-n}\right\} \\
& \times \exp \left\{-\sum_{n \geq 1} \frac{\omega^{-n}\left(P^{-n / 2}-P^{n / 2}\right)}{n\left(1-q^{n}\right)} z^{-n} \bar{\alpha}_{n}\right\}
\end{aligned}
$$

This network is unbalanced and, hence, produces wrong vertex operators $\widehat{V}^{\mathrm{Vir}}$, i.e. those which do not satisfy the usual commutation relations with the $q$-deformed Virasoro energy-momentum tensor. Notice also that the Heisenberg vertex operator $\widehat{V}^{\text {Heis }}$ is not the required Carlsson-Okounkov vertex operator [189, 190], i.e. the momenta are not shifted for the positive and negative modes (see also [186-188]).

\subsection{Network partition function}

Now we have all ingredients necessary for constructing network partition functions. It schematically has the form

$$
\left\langle\prod_{a} \Psi_{\lambda_{a}}\left[z_{a}\right] \prod_{b} \Psi_{\mu_{b}}^{*}\left[z_{b}^{*}\right] \prod_{c}\left(\sum_{\mu} \Psi_{\mu} \Psi_{\mu}^{*}\right)\right\rangle
$$

where the first product describes the external vertex operators, and the second one the "internal" screening operators. We denoted the vertex attached to brane $a$ by $\Psi_{a}$.

As we already mentioned at the end of the previous section, the deformation does not influence much the screening and vertex operators. This means that one can straightforwardly construct (3.36). Indeed, one can choose the normalization of the Heisenberg algebra operators in such a way that the pre-vertex operators become very simple:

$$
\begin{aligned}
C\{p\} & =\exp \left(\sum_{n>0} \frac{\mathfrak{a}_{n} p_{n}}{n}\right) & \bar{C}\{p\} & =\exp \left(-\sum_{n>0} \frac{\mathfrak{a}_{n} p_{n}}{n}\right) \\
C^{\dagger}\{p\} & =\exp \left(\sum_{n>0} \frac{\mathfrak{a}_{-n} p_{n}}{n}\right) & \bar{C}^{\dagger}\{p\} & =\exp \left(-\sum_{n>0} \frac{\mathfrak{a}_{-n} p_{n}}{n}\right)
\end{aligned}
$$

and the screening currents (3.18) get the non-deformed form

$$
S(x)=\exp \left\{-\sum_{n \geq 1} \frac{1}{n} x^{n} \tilde{\mathfrak{a}}_{-n}\right\} \exp \left\{\sum_{n \geq 1} \frac{1}{n} x^{-n} \tilde{\mathfrak{a}}_{n}\right\}
$$

In this simplified notation, the first part of formula (3.36), the external vertex operators, can be rewritten in the form (we are using equation (3.8) with the rescaled Heisenberg algebra)

$$
\prod_{I} \Psi_{\lambda_{I}}\left[z_{I}\right] \prod_{J} \Psi_{\mu_{J}}^{*}\left[z_{J}^{*}\right] \longrightarrow \prod_{I, J} \exp \left(\sum_{n \neq 0} \frac{1}{n}\left(\omega^{|n|}\left[\lambda_{I}, z_{I}\right]_{n} \mathfrak{a}_{n}-\left[\mu_{J}, z_{J}^{*}\right]_{n} \mathfrak{a}_{n}^{*}\right)\right)
$$


where all incoming vertex operators (labeled by the index $I$ ) are associated with the horizontal brane described by the Heisenberg operators $\mathfrak{a}_{n}$, while those outgoing ones (labeled by the index $J$ ) correspond to the Heisenberg operators $\mathfrak{a}_{n}^{*}$. Here the symbol

$$
[\lambda, z]_{n} \equiv \operatorname{sign}(n) \sum_{i}\left(q^{\lambda_{i}-1 / 2} t^{1 / 2-i} z\right)^{n}
$$

introduces the Miwa variables. This is exactly the formula (1.7).

Formulas (3.38) and (3.39) give simple expressions for the ingredients of (3.36), thus providing a description of the network partition functions.

As we already explained, in variance with vertex operators, the Virasoro/W-algebra non-trivially changes with deformation. We shall discuss this phenomenon in the next two sections, and here give a few examples of conformal blocks (calculated in terms of the non-rescaled Heisenberg algebras).

\subsection{Examples of conformal blocks}

The simplest conformal block $\mathcal{B}_{\mathbf{P G}}$. The simplest possible contraction corresponding to a nontrivial conformal block includes four intertwiners. It gives a peculiar "pure gauge" limit of the four-point Virasoro conformal block $\mathcal{B}_{4}\left(P_{1}, P_{2}, P_{3}, P_{4}, P, \mathfrak{x}\right)$, which, in the gauge theory language, corresponds to the pure $\mathrm{SU}(2)$ gauge theory partition function. In this limit [191], the dimensions $P_{i}$ of all the external fields become infinite, and simultaneously the points 0 and $\mathfrak{x}$ merge in a very particular way:

$$
P_{i} \rightarrow \infty, \quad \mathfrak{x} \rightarrow 0, \quad \mathfrak{x} P_{1} P_{2} P_{3} P_{4}=\Lambda^{4}=\text { fixed }
$$

Only two parameters, $\Delta$ and $\Lambda$ remain finite, so that $\mathcal{B}_{\mathrm{PG}}=\mathcal{B}_{\mathrm{PG}}(P, \Lambda)$.

The corresponding web partition function is equal to

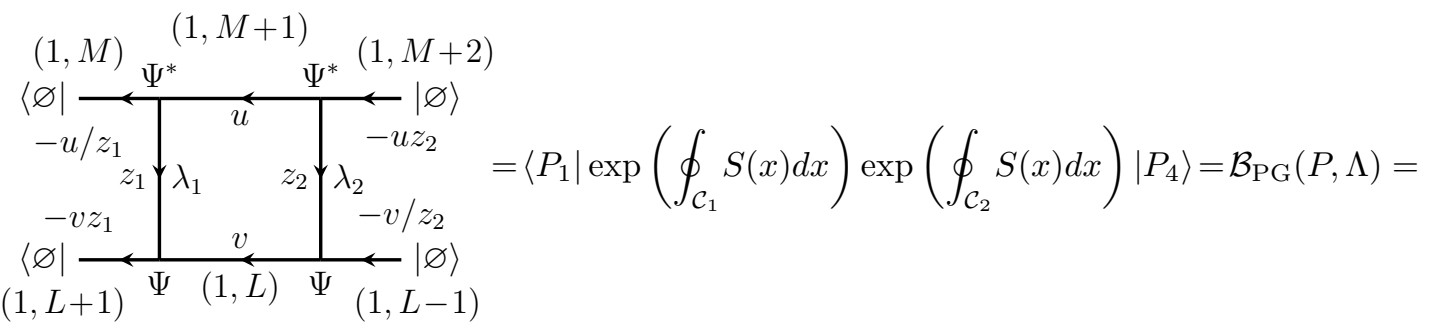

$$
\begin{aligned}
& =\sum_{\lambda_{1}, \lambda_{2}}\left(\frac{q v}{u}\left(-z_{1} z_{2}\right)^{\frac{M-L+1}{2}}\right)^{\left|\lambda_{1}\right|+\left|\lambda_{2}\right|}\left(-\frac{z_{1}}{z_{2}}\right)^{\frac{M-L+1}{2}\left(\left|\lambda_{1}\right|-\left|\lambda_{2}\right|\right)} \frac{f_{\lambda_{1}}^{M-L-1} f_{\lambda_{2}}^{M-L+1} q^{2 n\left(\lambda_{1}^{T}\right)+2 n\left(\lambda_{2}^{T}\right)}}{c_{\lambda_{1}} c_{\lambda_{2}} c_{\lambda_{1}}^{\prime} c_{\lambda_{2}}^{\prime}} \times
\end{aligned}
$$

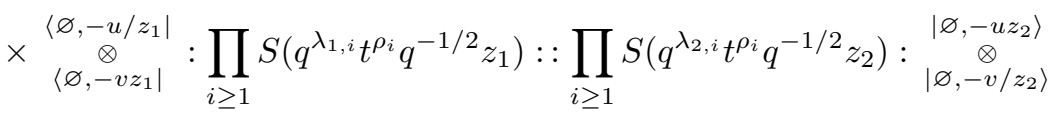

Here $\mathcal{C}_{1}$ and $\mathcal{C}_{2}$ are the contours encircling the two points 1 and $\Lambda . P_{1}$ and $P_{4}$ denote the momenta of the fields at points $0, \infty$. These momenta are actually infinite in the pure gauge limit. However, the infinite charges at zero and at infinity are compensated by the infinite number of screening charges coming from the two exponentials, so that the dimension of the field in the intermediate channel is finite and equal to $P$. In our formalism, $P$ is 
related to $\frac{u}{v}$ (since $u$ and $v$ are dimensions associated with the intermediate segments of the horizontal lines) and the $\Lambda=\frac{z_{1}}{z_{2}}$.

Using eq. (3.23), we can evaluate the matrix element of the two normal ordered factors in the last line of (3.42) to obtain the Vandermonde determinant:

$$
\left\langle\varnothing\left|\otimes\left\langle\varnothing\left|: \prod_{i \geq 1} S\left(q^{\lambda_{2, i}} t^{\rho_{i}} q^{-1 / 2} z_{2}\right):: \prod_{i \geq 1} S\left(q^{\lambda_{1, i}} t^{\rho_{i}} q^{-1 / 2} z_{1}\right):\right| \varnothing\right\rangle \otimes\right| \varnothing\right\rangle \sim \Delta^{(q, t)}(\{x\},\{y\})
$$

where

$$
x_{i}=q^{\lambda_{1, i}} t^{-i} z_{1}, \quad y_{i}=q^{\lambda_{2, i}} t^{-i} z_{2}
$$

Substituting the Vandermonde determinant back to eq. (3.42), one can verify that what is left is a particular limit of $(q, t)$-matrix model with the Chern-Simons terms:

$$
\begin{aligned}
& \mathcal{B}_{\mathrm{PG}}(P, \Lambda)= \\
& =\lim _{N_{1,2} \rightarrow \infty} \oint \oint d^{N_{1}} x d^{N_{2}} y \Delta^{(q, t)}(x) \Delta^{(q, t)}(y) \Delta^{(q, t)}(\{x\},\{y\}) \prod_{i=1}^{N_{1}} x_{i}^{\alpha_{1}} e^{(M-L+1) \frac{\left(\ln x_{i}\right)^{2}}{\ln q}} \prod_{j=1}^{N_{2}} y_{j}^{\alpha_{2}} e^{(M-L+1) \frac{\left(\ln y_{j}\right)^{2}}{\ln q}}
\end{aligned}
$$

where $q^{\alpha_{1,2}}=\frac{q v}{u} z_{1,2}^{M-L+1}$. The parameter $\Lambda=\frac{z_{1}}{z_{2}}$ is hidden inside the definition of the contour integrals $\mathcal{C}_{1,2}$. Notice that the Chern-Simons coupling constants depend on the relative slope of the two "horizontal" lines and, in particular, vanish for $L=M+1$, when we also have $\alpha_{1}=\alpha_{2}$.

Let us also give a spectral dual gauge theory interpretation for this conformal block. In the AGT correspondence, this limit of the conformal block corresponds to the pure SU(2) gauge theory, with $\Lambda$ being the instanton counting parameter (coupling constant) and $P$ being related to the Coulomb modulus $a$. After applying the spectral duality, however, we have a different interpretation: the coupling constant $\Lambda$ and the Coulomb modulus $q^{2 a}$ are exchanged. This spectral dual approach is directly applicable to eq. (3.42). If we simplify the infinite products (3.42) (or equivalently in the Vandermonde factors in eq. (3.45)) we get:

$$
\mathcal{B}_{\mathrm{PG}}(P, \Lambda)=\sum_{\lambda_{1}, \lambda_{2}}\left(\frac{q v}{u} z_{1}^{M-L+1}\right)^{\left|\lambda_{1}\right|}\left(\frac{q v}{u} z_{2}^{M-L+1}\right)^{\left|\lambda_{2}\right|}\left(f_{\lambda_{1}} f_{\lambda_{2}}\right)^{M-L+1} \frac{1}{z_{\mathrm{vec}}\left(\frac{z_{1}}{z_{2}}, \lambda_{1}, \lambda_{2}\right)}
$$

where $z_{\mathrm{vec}}$ is the standard Nekrasov factor. Notice that the whole sum becomes the Nekrasov function for the pure $\mathrm{SU}(2)$ theory (with additional "framing" factors in the case of general slopes $L \neq M+1$ ). However, the instanton counting parameter and the Coulomb modulus are related to $\frac{u}{v}$ and $\frac{z_{1}}{z_{2}}$ respectively, while, following the AGT duality it should be vice versa. Thus, what we write in eq. (3.46) is actually the spectral dual of the AGT dual Nekrasov function corresponding to the pure gauge limit of the conformal block.

Though this example is very simple in the gauge theory, as well as for the webs of intertwiners, from the point of view of the CFT it looks a bit contrived. The reason is that the corresponding diagram is not balanced. Let us describe a more regular example of a balanced diagram corresponding to a general four-point conformal block. 
More general Virasoro conformal blocks. To get the general four-point conformal block $\mathcal{B}_{4}$, we should combine the two balanced building blocks from eq. (3.29):

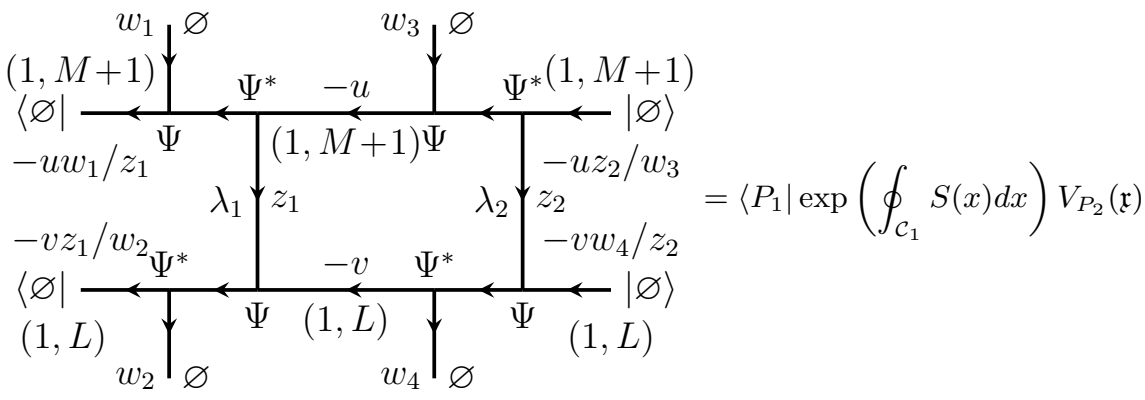

$$
\begin{aligned}
& \times \exp \left(\oint_{\mathcal{C}_{2}} S(x) d x\right) V_{P_{3}}(1)\left|P_{4}\right\rangle= \\
& =\mathcal{B}_{4}\left(P_{1}, P_{2}, P_{3}, P_{4}, P, \mathfrak{x}\right) \\
& =\sum_{\lambda_{1}, \lambda_{2}}\left(\frac{q v}{u}\left(-z_{1}\right)^{M-L+1}\right)^{\left|\lambda_{1}\right|}\left(\frac{q v w_{4} w_{3}}{u}\left(-z_{2}\right)^{M-L-1}\right)^{\left|\lambda_{2}\right|} \frac{f_{\lambda_{1}}^{M-L-1} f_{\lambda_{2}}^{M-L+1} q^{2 n\left(\lambda_{1}^{T}\right)+2 n\left(\lambda_{2}^{T}\right)}}{C_{\lambda_{1}} C_{\lambda_{2}} C_{\lambda_{1}}^{\prime} C_{\lambda_{2}}^{\prime}} \times
\end{aligned}
$$

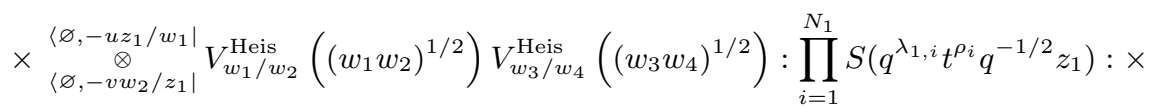

$$
\begin{aligned}
& \times V_{\left(t w_{2}\right) /\left(q w_{1}\right)}^{\mathrm{Vir}}\left(\left(w_{1} w_{2} q / t\right)^{1 / 2}\right): \prod_{i=1}^{N_{2}} S\left(q^{\lambda_{2, i}} t^{\rho_{i}} q^{-1 / 2} z_{2}\right): V_{\left(t w_{4}\right) /\left(q w_{3}\right)}^{\mathrm{Vir}}\left(\left(w_{3} w_{4} q / t\right)^{1 / 2}\right) \begin{array}{c}
\left|\varnothing,-u w_{3} / z_{2}\right\rangle \\
\varnothing \\
\left|\varnothing,-v w_{4} / z_{2}\right\rangle
\end{array}
\end{aligned}
$$

Here $t^{N_{1}}=\sqrt{\frac{t}{q}} \frac{z_{1}}{w_{1}}$ and $t^{N_{2}}=\sqrt{\frac{t}{q}} \frac{z_{2}}{w_{3}}$. Notice that the Heisenberg vertex operators commute with the Virasoro ones and also with the screening operators, so that their contribution factorizes and adds the standard "U(1) factor" to the conformal block. Employing the scaling invariance argument, one can consider only the conformal blocks in which the position of the last Virasoro vertex operator is the identity, so that $w_{3} w_{4} q / t=1$ and $w_{1} w_{2} q / t=\mathfrak{x}$. The dimensions of the primary fields are given by

$$
\begin{aligned}
P_{1} & =\frac{q v}{u}, \quad P_{2}=\frac{t w_{2}}{q w_{1}}, \quad P_{3}=\frac{w_{4}}{w_{3}}, \quad P_{4}=\frac{u z_{2}^{2}}{q v w_{4} w_{3}} \\
P & =\frac{t^{N_{1}+1} P_{2}}{q P_{1}}=\frac{t^{N_{2}} P_{4}}{P_{3}}
\end{aligned}
$$

The corresponding matrix model is of the Penner type with the additional Chern-Simons terms:

$$
\begin{aligned}
& \mathcal{B}_{4}\left(P_{1}, P_{2}, P_{3}, P_{4}, P, \mathfrak{x}\right)= \\
& =\oint \oint d^{N_{1}} x d^{N_{2}} y \Delta^{(q, t)}(x) \Delta^{(q, t)}(y) \Delta^{(q, t)}(\{x\},\{y\}) \prod_{i=1}^{N_{1}} x_{i}^{\alpha_{1}} e^{(M-L+1)} \frac{\left(\ln x_{i}\right)^{2}}{\ln q} \frac{\left(q^{1-\alpha_{2}} x_{i} / \mathfrak{x} ; q\right)_{\infty}}{\left(x_{i} / \mathfrak{x} ; q\right)_{\infty}} \frac{\left(q^{1-\alpha_{3}} x_{i} ; q\right)_{\infty}}{\left(x_{i} ; q\right)_{\infty}} \times \\
& \times \prod_{j=1}^{N_{2}} y_{j}^{\alpha_{4}} e^{(M-L+1) \frac{\left(\ln y_{j}\right)^{2}}{\ln q}} \frac{\left(q^{1-\alpha_{3}} y_{j} ; q\right)_{\infty}}{\left(y_{j} ; q\right)_{\infty}} \frac{\left(q^{1-\alpha_{2}} y_{j} / \mathfrak{x} ; q\right)_{\infty}}{\left(y_{j} / \mathfrak{x} ; q\right)_{\infty}}
\end{aligned}
$$

where $q^{\alpha_{i}}=P_{i}$.

The five-point conformal block can be obtained by putting three building blocks like (3.29) together. This gives a product of three Virasoro vertex operators, three Heisen- 
berg vertex operators and three groups of screening charges. Schematically, one has:

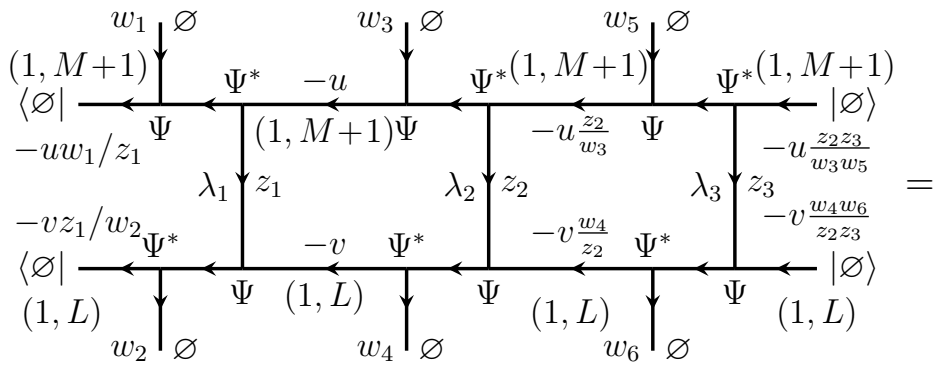

$$
\begin{aligned}
& =\left\langle P_{1}\left|\exp \left(\oint_{\mathcal{C}_{1}} S(x) d x\right) V_{P_{2}}\left(\mathfrak{x}_{1}\right) \exp \left(\oint_{\mathcal{C}_{2}} S(x) d x\right) V_{P_{3}}\left(\mathfrak{x}_{2}\right) \exp \left(\oint_{\mathcal{C}_{3}} S(x) d x\right) V_{P_{4}}(1)\right| P_{5}\right\rangle
\end{aligned}
$$

where $V_{P}$ includes both the Virasoro and Heisenberg parts.

Conformal blocks of $\boldsymbol{W}_{\boldsymbol{K}}$-algebra. Generalizing our formalism in another direction, we consider the $W_{3}$ algebra conformal block. In this case, there are three horizontal lines and two different types of screening currents $S_{1}$ and $S_{2}$, which correspond to the vertical segments between the first and second or the second and third lines respectively:

$$
S_{i}(x)=\exp \left\{-\sum_{n \geq 1} \frac{1}{n} \frac{1-t^{n}}{1-q^{n}}\left(1+\omega^{2 n}\right) x^{n} \tilde{\alpha}_{-n}^{(i)}\right\} \exp \left\{\sum_{n \geq 1} \frac{1}{n} \frac{1-t^{n}}{1-q^{n}}\left(1+\omega^{2 n}\right) x^{-n} \tilde{\alpha}_{n}^{(i)}\right\}
$$

where

$$
\begin{array}{cl}
\tilde{\alpha}_{n}^{(i)}=\frac{1}{1+\omega^{2 n}}\left(a_{n}^{(i)}-\omega^{n} a_{n}^{(i+1)}\right), & n \geq 1 \\
\tilde{\alpha}_{-n}^{(i)}=\frac{1}{1+\omega^{2 n}}\left(a_{-n}^{(i)}-\omega^{n} a_{-n}^{(i+1)}\right), \quad n \geq 1
\end{array}
$$

The simplest example is the pure gauge limit of the four-point block, which is given by the following web diagram:

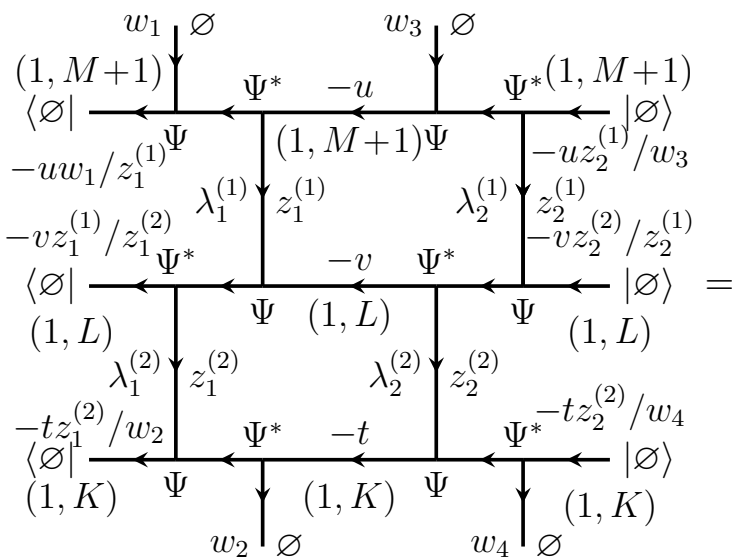

$$
\begin{aligned}
& =\left\langle\vec{P}_{1}\left|\exp \left(\oint_{\mathcal{C}_{1}} S_{1}(x) d x\right) \exp \left(\oint_{\mathcal{C}_{1}} S_{2}(x) d x\right) \exp \left(\oint_{\mathcal{C}_{2}} S_{1}(x) d x\right) \exp \left(\oint_{\mathcal{C}_{2}} S_{2}(x) d x\right)\right| \vec{P}_{4}\right\rangle
\end{aligned}
$$




\subsection{Compactified network and the affine screening operator}

Let us also give an expression for the compactified diagram describing the affine quiver gauge theory. The compactification identifies the vertical line going down the lower edge of the diagram with the line coming from the upper edge. Moreover, to get the general diagram, one should add one more ingredient, the shift in the spectral parameter. There is an automorphism of the DIM algebra, which multiplies the spectral parameters of all lines (and all elements of the algebra) by a constant. In general, the vertical compactification, i.e. the trace over vertical representation can contain a "twist" by this automorphism, which does not spoil the nice intertwining properties of the whole diagram. Taking the twist into account, one arrives at the "quasiperiodic" compactification, where the lines wrapping the compactification cylinder have their spectral parameters shifted. The whole picture now looks as follows:

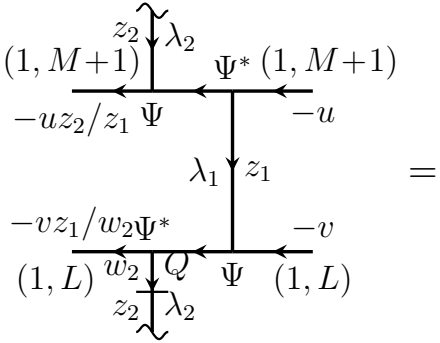

$$
\begin{aligned}
& =\sum_{\lambda_{1}, \lambda_{2}}\left(\frac{q v}{u}\left(-z_{1}\right)^{M-L+1}\right)^{\left|\lambda_{1}\right|}\left(\frac{q u w_{2}}{v z_{2}} Q\left(-z_{2}\right)^{L-M-1}\right)^{\left|\lambda_{2}\right|} \frac{f_{\lambda_{1}}^{M-L-1} f_{\lambda_{2}}^{M-L-1} q^{2 n\left(\lambda_{1}^{T}\right)+2 n\left(\lambda_{2}^{T}\right)}}{c_{\lambda_{1}} c_{\lambda_{1}}^{\prime} c_{\lambda_{2}} c_{\lambda_{2}}^{\prime}} \times \\
& \times: \prod_{i \geq 1} \widetilde{S}_{2}\left(q^{\lambda_{2, i}} t^{\rho_{i}} q^{-1 / 2} z_{2}\right):: \prod_{j \geq 1} S_{1}\left(q^{\lambda_{1, j}} t^{\rho_{j}} q^{-1 / 2} z_{1}\right):
\end{aligned}
$$

Here the wavy lines denote the identification of two vertical edges and the shift automorphism is marked by a short horizontal line. The automorphism shifts the spectral parameter of the line passing through it by $\frac{w_{2}}{z_{2}}$ and simultaneously adds $Q$ to the length of the corresponding edge. As in eq. (3.31) one has two types of screening operators $S_{1}$ and $\widetilde{S}_{2}$ corresponding to two simple roots of $\widehat{A}_{1}$, however because of the shift, the definition of the set of "root" oscillators inside the second screening is different:

$$
\begin{aligned}
\tilde{\alpha}_{n}^{(2)} & =\frac{1}{1+\omega^{2 n}}\left(a_{n}^{(2)}-\omega^{n}\left(z_{2} / w_{2}\right)^{n} a_{n}^{(1)}\right), & & n \geq 1 \\
\tilde{\alpha}_{-n}^{(2)} & =\frac{1}{1+\omega^{2 n}}\left(a_{-n}^{(2)}-\omega^{n}\left(w_{2} / z_{2}\right)^{n} a_{-n}^{(1)}\right), & & n \geq 1
\end{aligned}
$$

Taking an average, i.e. using the Wick theorem, one arrives at the affine $q$-Selberg matrix model [50]:

$$
\oint d^{N_{1}} x d^{N_{2}} y \frac{\Delta^{(q, t)}(x) \Delta^{(q, t)}(y)}{\Delta^{(q, t)}(\{x\},\{y\}) \Delta^{(q, t)}(\{y\},\{\tilde{t} x\})} \prod_{i=1}^{N_{1}} x_{i}^{\alpha_{1}} e^{(M-L+1) \frac{\left(\ln x_{i}\right)^{2}}{\ln q}} \prod_{j=1}^{N_{2}} y_{j}^{\alpha_{2}} e^{-(M-L+1) \frac{\left(\ln y_{j}\right)^{2}}{\ln q}}
$$

where the parameter of compactification $\tilde{t}=\sqrt{\frac{q}{t}} \frac{z_{2}}{w_{2}}, q^{\alpha_{1}}=\frac{q v}{u}, q^{\alpha_{2}}=Q \frac{q u}{v}$. Notice the characteristic combination of the Vandermonde factors in the measure, which is determined 
by the Cartan matrix of the affine algebra $\widehat{A}_{1}$. This matrix model can also be understood as the refined version of the ABJM matrix model [111-116], in particular, the level of the two Chern-Simons terms are opposite to each other. On the other hand, the spectral dual of this network is described by the elliptic DIM algebra (see appendix A2).

If one can cuts the diagram (3.55) along the vertical compactified line, one arrives at the regular Virasoro vertex operator (3.29). This is equivalent to the decompactification limit $Q \rightarrow \infty$, since in this case only $\lambda_{2}=\varnothing$ contributes.

\section{The action of Virasoro and $\operatorname{DIM}\left(\mathfrak{g l}_{1}\right)$}

There is a simple way [62] to build $q$-deformed Virasoro or $W_{K}$-algebras from DIM generators. To this end, one considers the dressed current $t(z)$ :

$$
t(z)=\alpha(z) x^{+}(z) \beta(z)
$$

where

$$
\alpha(z)=\exp \left(-\sum_{n \geq 1} \frac{1}{\gamma^{n}-\gamma^{-n}} b_{-n} z^{n}\right), \quad \beta(z)=\exp \left(\sum_{n \geq 1} \frac{1}{\gamma^{n}-\gamma^{-n}} b_{n} z^{-n}\right)
$$

and $b_{n}$ are the modes of the $\psi^{ \pm}$generators:

$$
\psi^{ \pm}(z)=\psi_{0}^{ \pm} \exp \left( \pm \sum_{n \geq 1} b_{ \pm n} \gamma^{n / 2} z^{\mp n}\right)
$$

The dressing is needed to kill the extra Heisenberg part of the algebra. The element $t(z)$ acts as a $W_{K}$ algebra current in the $K$-fold tensor product of Fock modules $\mathcal{F}_{u_{1}} \otimes \cdots \otimes \mathcal{F}_{u_{K}}$. One has:

$$
\rho_{u_{1}, \ldots, u_{K}}(t(z))=\sum_{i=1}^{K} u_{i} \Lambda_{i}(z)
$$

where

$$
\Lambda_{i}(z)=\exp \left(\sum_{n \geq 1} \frac{1-t^{-n}}{n} z^{n} \hat{\alpha}_{-n}^{(i)}\right) \exp \left(-\sum_{n \geq 1} \frac{1-t^{n}}{n} z^{-n} \hat{\alpha}_{n}^{(i)}\right)
$$

The oscillators $\hat{\alpha}_{n}^{(i)}$ are defined as

$$
\hat{\alpha}_{n}^{(i)}=\hat{\hat{\alpha}}_{n}^{(i)}-\bar{\alpha}_{n}
$$

where (compare with [72, s.4.2]

$$
\begin{array}{ll}
\hat{\hat{\alpha}}_{n}^{(i)}=a_{n}^{(i)} \omega^{(i-1) n}, & n \geq 1, \\
\hat{\hat{\alpha}}_{-n}^{(i)}=\left(1-\omega^{-2 n}\right)\left(a_{-n}^{(1)}+a_{-n}^{(2)} \omega^{-n}+\ldots+a_{-n}^{(i-1)} \omega^{(2-i) n}\right)+a_{-n}^{(i)} \omega^{(1-i) n}, & n \geq 1
\end{array}
$$

and the Heisenberg part oscillators are given by

$$
\bar{\alpha}_{n}=\frac{1-\omega^{-2 n}}{1-\omega^{-2 n K}}\left(a_{n}^{(1)}+a_{n}^{(2)} \omega^{-n}+\ldots+a_{n}^{(K)} \omega^{(1-K) n}\right), \quad n \geq 1,
$$




$$
\bar{\alpha}_{-n}=\frac{1-\omega^{-2 n}}{1-\omega^{-2 n K}}\left(a_{-n}^{(1)}+a_{-n}^{(2)} \omega^{-n}+\ldots+a_{-n}^{(K)} \omega^{(1-K) n}\right), \quad n \geq 1,
$$

Here $a_{n}^{(i)}$ acts in the $i$-th Fock module. Notice that we can obtain the "root" bosons $\tilde{\alpha}_{n}^{(i)}$ from $\hat{\alpha}_{n}^{(i)}$ :

$$
\begin{aligned}
\tilde{\alpha}_{n}^{(i)} & =\frac{\omega^{(i+1) n}}{1+\omega^{2 n}}\left(\hat{\alpha}_{n}^{(i)}-\hat{\alpha}_{n}^{(i+1)}\right), \\
\tilde{\alpha}_{-n}^{(i)} & =\frac{\omega^{(1-i) n}}{1+\omega^{2 n}}\left(\hat{\alpha}_{-n}^{(i)}-\hat{\alpha}_{-n}^{(i+1)}\right)
\end{aligned}
$$

Another useful property of the oscillators $\hat{\alpha}_{n}^{(i)}$ is that they commute with the "U(1)" oscillators $\bar{\alpha}_{n}$ :

$$
\left[\hat{\alpha}_{n}, \bar{\alpha}_{m}\right]=0 .
$$

The $W_{K}$ algebra is built from the generators $\Lambda_{i}(z)$ by the Miura transform:

$$
W_{k+1}(z)=\sum_{i_{1}<\ldots<i_{k+1}} u_{i_{1}} \cdots u_{i_{k+1}}: \Lambda_{i_{1}}(z) \Lambda_{i_{2}}\left(\omega^{2} z\right) \cdots \Lambda_{i_{k+1}}\left(\omega^{2 k} z\right):
$$

The screening charges are built from the contractions of the DIM intertwiners. Thus, they commute with any element of the DIM algebra, e.g. with $t(z)$ by construction. This returns us to the definition of the Virasoro algebra as the centralizer of the screening charges [50]. Any element of the DIM algebra acts in the tensor products of some of the representations corresponding to the lines of the network. This can be described as an action in a particular section of the diagram (see figure 1). The DIM element acts in the tensor product of Fock modules associated with the legs intersected by the dotted line. The section can be brought through the intertwiners, so that eventually the element of the algebra acts on the external lines. These external lines correspond to the vertex operators, and the commutation with the intertwiners leads to the Ward identities for the corresponding CFT or matrix model.

One should always be careful to include all the spaces, which are intersected by the section. Let us give an example of commutation of the DIM element with the contraction of two vertices. Pictorially we have:

$$
\rho_{-u y}^{(1, M)} \otimes \rho_{-\frac{v}{y}}^{(1, L+1)} \Delta\left(x^{ \pm}(z)\right) \quad \rho_{u}^{(1, M+1)} \otimes \rho_{v}^{(1, L)} \Delta\left(x^{ \pm}(z)\right)
$$

This can be written out as follows:

$$
\left[\rho_{u_{1}} \otimes \rho_{u_{2}}\left(\Delta\left(x^{ \pm}(\omega z)\right)\right), \sum_{\lambda}\left\|M_{\lambda}\right\|^{-2}\left(\begin{array}{c}
\Psi_{\lambda}^{*}(y) \\
\otimes \\
\Psi_{\lambda}(y)
\end{array}\right)\right]
$$




$$
\begin{gathered}
=\left[\rho_{u_{1}}\left(x^{ \pm}\left(\omega^{\xi_{ \pm}} z\right)\right) \otimes \rho_{u_{2}}\left(\Xi^{-\xi_{\mp}}(z)\right), \sum_{\lambda}\left\|M_{\lambda}\right\|^{-2}\left(\begin{array}{c}
\Psi_{\lambda}^{*}(y) \\
\otimes \\
\Psi_{\lambda}(y)
\end{array}\right)\right]+ \\
+\left[\rho_{u_{1}}\left(\Xi^{\xi_{ \pm}}(z)\right) \otimes \rho_{u_{2}}\left(x^{ \pm}\left(\omega^{\xi_{\mp}} z\right)\right), \sum_{\lambda}\left\|M_{\lambda}\right\|^{-2}\left(\begin{array}{c}
\Psi_{\lambda}^{*}(y) \\
\otimes \\
\Psi_{\lambda}(y)
\end{array}\right)\right]= \\
=(\omega / q)^{\xi_{\mp}} \sum_{\lambda} \frac{q}{\left\|M_{\lambda}\right\|^{2}} \sum_{i=1}^{l(\lambda)} A_{\lambda, i}^{-} \delta\left(x_{i} t y /(q z)\right)\left(\begin{array}{c}
\rho_{u_{1}}\left(\Xi^{\xi_{ \pm}}(z)\right) \Psi_{\lambda-\xi_{ \pm} \cdot 1_{i}}^{*}(y) \\
\otimes \\
\Psi_{\lambda-\xi_{\mp} \cdot 1_{i}}(y) \rho_{u_{2}}\left(\Xi^{-\xi_{\mp}}(z)\right)
\end{array}\right)+ \\
+(\omega / q)^{\xi_{\mp}} \sum_{\lambda} \frac{1}{\left\|M_{\lambda}\right\|^{2}} \sum_{i=1}^{l(\lambda)+1} A_{\lambda, i}^{+} \delta\left(x_{i} t y / z\right)\left(\begin{array}{c}
\rho_{u_{1}}\left(\Xi^{\xi_{ \pm}}(z)\right) \Psi_{\lambda+\xi_{\mp} \cdot 1_{i}}^{*}(y) \\
\otimes \\
\Psi_{\lambda+\xi_{ \pm} \cdot 1_{i}}(y) \rho_{u_{2}}\left(\Xi^{-\xi_{\mp}}(z)\right)
\end{array}\right)= \\
\left.=(\omega / q)^{\xi_{\mp}} \sum_{\lambda} \sum_{i=1}^{l(\lambda)+1}\left(\frac{q A_{\lambda+1_{i}, i}^{-}}{\left\|M_{\lambda+1_{i}}\right\|^{2}}+\frac{A_{\lambda, i}^{+}}{\left\|M_{\lambda}\right\|^{2}}\right) \begin{array}{c}
A_{u_{1}}\left(\Xi_{i} t y / z\right) \\
\left.\Xi_{ \pm}(z)\right) \Psi_{\lambda+\xi_{\mp} \cdot 1_{i}}^{*}(y) \\
\otimes \\
\Psi_{\lambda+\xi_{ \pm} \cdot 1_{i}}(y) \rho_{u_{2}}\left(\Xi^{\left.-\xi_{\mp}(z)\right)}\right.
\end{array}\right)=0
\end{gathered}
$$

where

$$
\begin{aligned}
A_{\lambda, i}^{+} & =(1-t) \prod_{j=1}^{i-1} \frac{\left(1-t \frac{x_{i}}{x_{j}}\right)\left(1-\frac{q}{t} \frac{x_{i}}{x_{j}}\right)}{\left(1-\frac{x_{i}}{x_{j}}\right)\left(1-q \frac{x_{i}}{x_{j}}\right)} \\
A_{\lambda, i}^{-} & =\left(1-t^{-1}\right) \prod_{j=i+1}^{\infty} \frac{\left(1-\frac{t}{q} \frac{x_{i}}{x_{j}}\right)\left(1-\frac{1}{t} \frac{x_{i}}{x_{j}}\right)}{\left(1-\frac{1}{q} \frac{x_{i}}{x_{j}}\right)\left(1-\frac{x_{i}}{x_{j}}\right)} \\
\xi_{+} & =1, \quad \xi_{-}=0, \quad \Xi^{ \pm 1}(y)=\psi^{\mp}\left(\omega^{1 / 2} y\right), \quad \Xi^{0}(y)=1, \quad x_{i}=q^{\lambda_{i}} t^{-i}
\end{aligned}
$$

and we remind that $\omega=\sqrt{q / t}$. The last line in eq. (4.15) vanishes because of a particular sum rule for the norms of Macdonald polynomials. Commutation with $\psi^{ \pm}(z)$ can also be explicitly verified:

$$
\begin{aligned}
& \rho_{u_{1}} \otimes \rho_{u_{2}} \Delta\left(\psi^{ \pm}(y)\right) \sum_{\lambda}\left\|M_{\lambda}\right\|^{-2}\left(\begin{array}{c}
\Psi_{\lambda}^{*}(z) \\
\otimes \\
\Psi_{\lambda}(z)
\end{array}\right)=\sum_{\lambda}\left\|M_{\lambda}\right\|^{-2}\left(\begin{array}{c}
\rho_{u_{1}}\left(\psi^{ \pm}\left(\omega^{\mp 1 / 2} y\right)\right) \Psi_{\lambda}^{*}(z) \\
\otimes \\
\rho_{u_{2}}\left(\psi^{ \pm}\left(\omega^{ \pm 1 / 2} y\right)\right) \Psi_{\lambda}(z)
\end{array}\right)= \\
= & \sum_{\lambda}\left\|M_{\lambda}\right\|^{-2}\left(\begin{array}{c}
\Psi_{\lambda}^{*}(z) \rho_{u_{1}}\left(\psi^{ \pm}\left(\omega^{\mp 1 / 2} y\right)\right) \\
\otimes \\
\Psi_{\lambda}(z) \rho_{u_{2}}\left(\psi^{ \pm}\left(\omega^{ \pm 1 / 2} y\right)\right)
\end{array}\right)=\sum_{\lambda}\left\|M_{\lambda}\right\|^{-2}\left(\begin{array}{c}
\Psi_{\lambda}^{*}(z) \\
\otimes \\
\Psi_{\lambda}(z)
\end{array}\right) \rho_{u_{1}} \otimes \rho_{u_{2}} \Delta\left(\psi^{ \pm}(y)\right)
\end{aligned}
$$

All the commutation calculations above work by a similar mechanism, summarized schematically in figure 1, b). The action of the DIM element on the two horizontal representations is first transformed into its action on the intermediate vertical segment and finally the other side of the dashed line is also pulled through the vertex to get the commutation.

The action of the element $t(z)$ gives the Ward identities of the corresponding matrix model. This can be seen directly by computing the operator product expansion of this current with the screening charges. For example in the Virasoro case (see [50] for details):

$$
\rho_{u_{1}} \otimes \rho_{u_{2}}(\Delta(t(z))) S(y)=\frac{1-t \frac{y}{z}}{1-\frac{y}{z}}: u_{1} \Lambda_{1}(z) S(y):+t \frac{1-\frac{q}{t} \frac{y}{z}}{1-q \frac{y}{z}}: u_{2} \Lambda_{2}(z) S(y):
$$


Since $t(z)$ commutes with the screening charges, in any correlator with these latter it can be brought through to the vacuum, which is annihilated by the negative modes of $t(z)$. Following this logic, one gets the matrix model Ward identities, the regularity of certain averages:

$$
\left\langle K_{+}(z) \prod_{i} \frac{1-t \frac{x_{i}}{z}}{1-\frac{x_{i}}{z}}+K_{-}(z) \prod_{i} \frac{1-\frac{q}{t} \frac{x_{i}}{z}}{1-q \frac{x_{i}}{z}}\right\rangle=\operatorname{Regular}(z)
$$

where $K_{ \pm}(z)$ are certain polynomials. This equation is the appropriate $(q, t)$-deformations of the familiar Ward identity (1.5).

\section{Vertical action of DIM}

As was mentioned earlier, the vertical representation of the DIM algebra has a combinatorial description in terms of Young diagrams, [60,63]. We have

$$
\begin{aligned}
\rho_{u}^{(0,1)}\left(x^{+}(z)\right)\left|M_{\lambda}^{(q, t)}\right\rangle & =\sum_{i=1}^{l(\lambda)+1} A_{\lambda, i}^{+} \delta\left(x_{i} t u / z\right)\left|M_{\lambda+1_{i}}^{(q, t)}\right\rangle \\
\rho_{u}^{(0,1)}\left(x^{-}(z)\right)\left|M_{\lambda}^{(q, t)}\right\rangle & =\omega \sum_{i=1}^{l(\lambda)} A_{\lambda, i}^{-} \delta\left(x_{i} t u /(q z)\right)\left|M_{\lambda-1_{i}}^{(q, t)}\right\rangle \\
\rho_{u}^{(0,1)}\left(\psi^{+}(z)\right)\left|M_{\lambda}^{(q, t)}\right\rangle & =\omega B_{\lambda}^{+}(u / z)\left|M_{\lambda}^{(q, t)}\right\rangle \\
\rho_{u}^{(0,1)}\left(\psi^{-}(z)\right)\left|M_{\lambda}^{(q, t)}\right\rangle & =\omega^{-1} B_{\lambda}^{-}(z / u)\left|M_{\lambda}^{(q, t)}\right\rangle
\end{aligned}
$$

where $A_{\lambda, i}^{ \pm}$were defined in eq. (4.16) and

$$
\begin{aligned}
& B_{\lambda}^{+}(z)=\prod_{i=1}^{\infty} \frac{1-z x_{i}}{1-t z x_{i}} \frac{1-\frac{t^{2}}{q} z x_{i}}{1-\frac{t}{q} z x_{i}} \\
& B_{\lambda}^{-}(z)=\prod_{i=1}^{\infty} \frac{1-\frac{z}{x_{i}}}{1-\frac{z}{t x_{i}}} \frac{1-\frac{q}{t^{2}} \frac{z}{x_{i}}}{1-\frac{q}{t} \frac{z}{t x_{i}}}
\end{aligned}
$$

where $x_{i}=q^{\lambda_{i}} t^{-i}$.

Similar action in the Yangian limit $q, t \rightarrow 1$ has been considered in [49], where the matrix model Ward identities or regularity condition for $q q$-characters (4.21) were derived from the intertwining property with the Virasoro vertex operators. We should note here that the interpretation of the vertex operators in this work was spectrally dual to our present consideration, i.e. the $\mathrm{SU}(N)$ gauge theory corresponded there to the four-point conformal block of the $W_{N}$ algebra as prescribed by the AGT relation.

In our formalism such an intertwining relation is natural: the vertex operator is build out of the DIM intertwiners, which combinations commute with elements of DIM. However, this is only true unless there are external legs. If we consider a horizontal section of the web diagram, and try to move it between the "layers" of the diagram, we necessarily encounter the external legs, or vertex operators in the language of [49]. The DIM generators do not commute with the intertwiners having external legs, since one should consider the 
additional terms due to the action of DIM element on these legs. However, these terms turn out to be tractable. Indeed, they precisely reproduce the $q q$-character insertion into the matrix model average. The matrix model arises from the sum over diagrams residing on the legs intersected by the horizontal section.

\section{Spectral duality and change of basis}

In $[31,32]$ it was shown that the change of preferred direction in refined topological string is a nontrivial change of basis. The change of basis is nontrivial in the sense that for the states on several parallel legs the matrix of this transformation does not factorize into a tensor product of matrices acting on each leg. Indeed, the transformation is given by the spectral duality, and the two basis sets are the standard Schur (or Macdonald) symmetric functions and the generalized Macdonald polynomials [41, 192-195]. This matrix was called generalized Kostka function in [31, 32].

In this subsection, we show how the spectral duality shows up in our present algebraic approach. Let us consider the "vertical" basis in the tensor product of vertical representations $\rho_{u_{1}, \ldots, u_{K}}^{(0,1)}=\rho_{u_{1}}^{(0,1)} \otimes \cdots \otimes \rho_{u_{K}}^{(0,1)}$, which we define as the set of eigenvalues of the particular DIM element $\psi_{-1}^{+}$:

$$
\begin{aligned}
& \rho_{u_{1}, \ldots, u_{K}}^{(0,1)} \Delta^{K-1} \psi_{-1}^{+}\left|M_{\lambda_{1}}^{(q, t)}\right\rangle \otimes \cdots \otimes\left|M_{\lambda_{K}}^{(q, t)}\right\rangle \\
& =(1-t)(1-t / q) \sum_{k=1}^{K}\left(u_{k} \sum_{i=1}^{\infty} q^{\lambda_{k, i}} t^{-i}\right)\left|M_{\lambda_{1}}^{(q, t)}\right\rangle \otimes \cdots \otimes\left|M_{\lambda_{K}}^{(q, t)}\right\rangle
\end{aligned}
$$

where $\psi_{-1}^{+}=\oint \psi^{+}(z) d z$. For generic $u_{i}$, all eigenvalues are distinct, so this property defines the basis uniquely. This basis is certainly very simple: it is a tensor product of Macdonald polynomials.

Let us now perform the spectral duality. In the DIM algebra, this corresponds to the $S$-transformation from $\mathrm{SL}(2, \mathbb{Z})$ acting on the generators of the algebra. Under this transformation, $\psi_{-1}^{+}$transforms into $x_{0}^{+}=\oint x^{+}(z) d z / z$. The basis (5.7) transforms into the basis of $x_{0}^{+}$with the same eigenvalues. However, the operator $x_{0}^{+}$should be taken in the new representation $\rho_{u_{1}, \ldots, u_{M}}^{(1,0)}=\rho_{u_{1}}^{(1,0)} \otimes \cdots \otimes \rho_{u_{K}}^{(1,0)}$ :

$$
\begin{aligned}
& \rho_{u_{1}, \ldots, u_{K}}^{(1,0)} \Delta^{K-1} x_{0}^{+}\left|M_{\lambda_{1}, \ldots, \lambda_{K}}^{(q, t)}\left(u_{1}, \ldots, u_{K}\right)\right\rangle \\
& =(1-t)(1-t / q) \sum_{k=1}^{K}\left(u_{k} \sum_{i=1}^{\infty} q^{\lambda_{k, i}} t^{-i}\right)\left|M_{\lambda_{1}, \ldots, \lambda_{K}}^{(q, t)}\left(u_{1}, \ldots, u_{K}\right)\right\rangle
\end{aligned}
$$

We recognize the operator $\rho_{u_{1}, \ldots, u_{K}}^{(1,0)} \Delta^{K-1} x_{0}^{+}$: this is just the generalized Macdonald Hamiltonian. The eigenvalues also match, so the new basis $\left|M_{\lambda_{1}, \ldots, \lambda_{K}}^{(q, t)}\left(u_{1}, \ldots, u_{K}\right)\right\rangle$ is the basis of generalized Macdonald polynomials. The generalized Kostka functions are just representations of this $\mathrm{SL}(2, \mathbb{Z})$ transformation.

\section{Conclusion}

In this paper, we presented technical details on evaluation of the Nekrasov functions and their symmetries (including the $q q$-character correlators) from the free field formalism for 
the $\operatorname{DIM}\left(g l_{1}\right)$ algebra. This is a very powerful method, but it is only at the first stage of development. There are several restrictions which should be consequently lifted at the next stages. If one considers them as a consequent specification of representation types, the list should be read in inverse order.

- The construction that admits fermionization of intertwiners $\Psi$ and $\Psi^{*}$ at the level one of DIM is much similar to that for the level $k=1$ Kac-Moody algebras. Hence, one could expect a straightforward generalization to arbitrary level a la [95-97] involving analogues of the $b, c$-systems. Note, however, that the requirement on the level does not restrict the value of the Virasoro central charge regulated by $\beta$ : all matrix models and $\beta$-ensembles and, hence, the generic Liouville and $W_{K}$-conformal blocks are already handled by the existing formalism. Also, at this level the difference disappears between the vertex operators (in particular, the screening charges) and the stress tensors (including the $\mathrm{W}$-operators): all these are described by exponentials of the free fields, the differences emerge only in the limit $q, t \longrightarrow 1$.

- The formalism is best developed for the intertwiners, which act as operators between the two "horizontal" Fock modules $\mathcal{F}^{(1, L)}$ and $\mathcal{F}^{(1, L \pm 1)}$, while the third representation is the "vertical" leg associated with $\mathcal{F}^{(0, \pm 1)}$. Such a non-symmetricity is inevitable since the resulting topological vertex of [35] is still asymmetric and remembers about the distinguished vertical direction. Technically this restricts consideration to the balanced networks, what makes many important models, including the quiver ones, treatable only via additional application of the spectral duality.

- A better treatment should involve infinitely many free fields, giving rise to MacMahon type modules, what should also allow one to define skew intertwiners, where all the three legs are non-vertical. An existing description of the MacMahon modules is pure combinatorial, in terms of $3 d$ Young diagrams (plane partitions). A naive free field formalism would involve fields depending on two coordinates instead of one, and this requires a far-going generalization of holomorphic fields used in the ordinary $2 d$ CFT. Such a formalism is now developing, also with the motivation coming from MHV amplitudes, but its incorporation into the DIM representation theory is a matter of future. Still, it seems important for a full understanding of the spectral dualities and of generic networks, including the sophisticated ones from [165-169]. They can be treated by the existing formalism, but it leaves the underlying symmetries well hidden: they show up only in answers, but not at any of the intermediate stages.

- A further challenge is further generalization from $\operatorname{DIM}\left(\mathfrak{g l}_{1}\right)$ to $\operatorname{DIM}\left(\mathfrak{g l}_{n}\right)$ and the triple-Pagoda algebras $\operatorname{DIM}\left(\widehat{\mathfrak{g l}_{1}}\right)$ and $\operatorname{DIM}\left(\widehat{\mathfrak{g l}_{n}}\right)$. An intriguing problem (see appendix A3) is that already $\operatorname{DIM}\left(\mathfrak{g l}_{n}\right)$ is built from the affine Dynkin diagram of $\widehat{\mathfrak{g l}}_{n}$, thus, the triple-affine generalization should involve more sophisticated Dynkin diagrams.

We hope that the present text can serve as a good introduction in the DIM-based generalization of conformal theories, where the conformal blocks are the generic Nekrasov functions and the Ward identities are the associated regularity conditions for $q q$-characters. 
We hope that it will help to attract more attention to emerging challenging problems, which we have just enumerated. Technical means for this seem to be already at hand.

\section{Acknowledgments}

A.M.'s and Y.Z. are grateful for remarkable hospitality at Nagoya University during the work on this project.

Our work is supported in part by Grant-in-Aid for Scientific Research (\# 24540210) (H.A.), (\# 15H05738) (H.K.), for JSPS Fellow (\# 26-10187) (Y.O.), JSPS Grant-in-Aid for Young Scientists (B) \# 16K17567 (T.M.) and JSPS Bilateral Joint Projects (JSPSRFBR collaboration) "Exploration of Quantum Geometry via Symmetry and Duality" from MEXT, Japan. It is also partly supported by grants 15-31-20832-Mol-a-ved (A.Mor.), 15-31-20484-Mol-a-ved (Y.Z.), mol-a-dk 16-32-60047 (And.Mor), by RFBR grants 16-0100291 (A.Mir.) and 16-02-01021 (A.Mor. and Y.Z.), by joint grants 15-51-50034-YaF, 15-51-52031-NSC-a, 16-51-53034-GFEN.

\section{A Properties of the DIM algebras and their limits}

In this appendix, we describe the algebraic structures of DIM algebras and their degenerations.

\section{A.1 Constructing $\operatorname{DIM}\left(\mathfrak{g l}_{1}\right)$ from $W_{1+\infty}$ algebra}

Let us discuss how one can construct $\operatorname{DIM}\left(\mathfrak{g l}_{1}\right)$ starting from the algebra of difference operators, $[55,196]$.

Algebra $\boldsymbol{W}_{\mathbf{1 + \infty}}$. Consider the algebra $W_{1+\infty}$ (as usual, $1+\infty$ refers here to adding the Heisenberg algebra to $\left.W_{\infty}\right)$ given by the generators $W_{n}^{k}=W\left(z^{n} \mathcal{D}^{k}\right), n \in \mathbb{Z}, k \in \mathbb{Z}_{\geq 0}$, where $\mathcal{D}=z \partial_{z}$. One can consider the central extension of this algebra:

$$
\begin{aligned}
{\left[W\left(z^{n} \mathcal{D}^{k}\right), W\left(z^{m} \mathcal{D}^{l}\right)\right] } & =W\left(\left[z^{n} \mathcal{D}^{k}, z^{m} \mathcal{D}^{l}\right]\right)+c \delta_{n+m, 0} \cdot \psi_{n, k l}, \\
\psi_{n, k l} & =\left\{\begin{array}{cl}
\sum_{j=1}^{n}(-j)^{k}(n-j)^{l}, & n>0 \\
0 & n=0
\end{array}\right.
\end{aligned}
$$

or, in the different basis of $W_{n}^{k}=W\left(z^{n} D^{k}\right)$ with $D \equiv \mathfrak{t}^{\mathcal{D}}$ (see (A.22)),

$$
\left[W\left(z^{n} D^{k}\right), W\left(z^{m} D^{l}\right)\right]=\left(\mathfrak{t}^{m k}-\mathfrak{t}^{n l}\right) W\left(z^{n+m} D^{k+l}\right)-c \delta_{n+m, 0} \frac{\mathfrak{t}^{m k}-\mathfrak{t}^{n l}}{\mathfrak{t}^{k+l}-1}
$$

Note that, if $k+l \neq 0$, the second term in the right hand side of (A.2) can be absorbed into the first term by redefining the generators $W\left(\mathcal{D}^{k}\right)$ with $k \neq 0: W\left(D^{k}\right) \rightarrow W\left(D^{k}\right)-$ $\frac{c}{\mathfrak{t}^{k}-1}, k \neq 0$. However, at $k+l=0$ this term can not be absorbed and is equal to $n c t^{-n k} \delta_{n+m, 0} \delta_{k+l, 0}$, see (A.3). 
Algebra $\overline{\boldsymbol{W}_{\mathbf{1 + \infty}}}$. The next step is to consider the algebra $\overline{W_{1+\infty}}=\left\{W\left(z^{n} q^{k D}\right), n, k \in\right.$ $\mathbb{Z}\}$, which is a double of the $W_{1+\infty}$ and may have two central extensions:

$$
\left[W\left(z^{n} D^{k}\right), W\left(z^{m} D^{l}\right)\right]=\left(\mathfrak{t}^{m k}-\mathfrak{t}^{n l}\right) W\left(z^{n+m} D^{k+l}\right)+\mathfrak{t}^{-n k}\left(n c_{1}+k c_{2}\right) \delta_{m+n, 0} \delta_{k+l, 0}
$$

Automorphisms. The algebra $\overline{W_{1+\infty}}$, (A.3) has the evident automorphisms $\sigma, \tilde{\sigma}$ and $\tau$ defined by

$$
\begin{array}{lll}
\sigma\left(W_{n}^{k}\right)=\mathfrak{t}^{-n k} W_{k}^{-n}, & \sigma\left(c_{1}\right)=-c_{2}, & \sigma\left(c_{2}\right)=c_{1}, \\
\tilde{\sigma}\left(W_{n}^{k}\right)=-W_{k}^{n}, & \tilde{\sigma}\left(c_{1}\right)=c_{2}, & \tilde{\sigma}\left(c_{2}\right)=c_{1}, \\
\tau\left(W_{n}^{k}\right)=\mathfrak{t}^{\frac{1}{2} n^{2}} W_{n}^{k+n}, & \tau\left(c_{1}\right)=c_{1}+c_{2}, & \tau\left(c_{2}\right)=c_{2} .
\end{array}
$$

In particular, $\sigma$ and $\tau$ form $\mathrm{SL}(2, \mathbb{Z})$ acting on two central charges $c_{1}$ and $c_{2}$.

Heisenberg subalgebras. By the commutation relations (A.3), it is easy to see that it contains a Heisenberg subalgebra generated by $\left\{W_{n}^{0}, c_{1}\right\}_{n \in \mathbb{Z}}$ satisfying

$$
\left[W_{n}^{0}, W_{m}^{0}\right]=n c_{1} \delta_{n+m, 0} .
$$

From the viewpoint of the root lattice of $\overline{W_{1+\infty}}$, this can be seen as the vertical embedding of the Heisenberg algebra. By using the automorphisms $\sigma$ and $\tau$ in the above, it is easy to find the horizontal and the embedding with arbitrary slope $\alpha \in \mathbb{Z}$ as follows;

$$
\begin{aligned}
{\left[W_{0}^{n}, W_{0}^{m}\right] } & =n c_{2} \delta_{n+m, 0}, \\
{\left[W_{n}^{\alpha n}, W_{m}^{\alpha m}\right] } & =n \mathfrak{t}^{-\alpha n^{2}}\left(c_{1}+\alpha c_{2}\right) \delta_{n+m, 0} .
\end{aligned}
$$

Chevalley generators and Serre relations. The generators $W_{n}^{ \pm, 0}=W\left(z^{n} D^{ \pm 1,0}\right)$ form a closed subalgebra:

$$
\begin{aligned}
{\left[W_{n}^{+}, W_{m}^{-}\right] } & =\left(\mathfrak{t}^{m}-\mathfrak{t}^{-n}\right) W_{m+n}^{0}+\left(n c_{1}+c_{2}\right) \mathfrak{t}^{-n} \delta_{n+m, 0} \\
{\left[W_{n}^{0}, W_{m}^{ \pm}\right] } & =\left(1-\mathfrak{t}^{ \pm n}\right) W_{m+n}^{ \pm} \\
{\left[W_{n}^{0}, W_{m}^{0}\right] } & =n c_{1} \delta_{n+m, 0}
\end{aligned}
$$

One can generate the whole algebra from this subalgebra provided the Serre relations are added:

$$
\left[W_{n}^{ \pm},\left[W_{n+1}^{ \pm}, W_{n-1}^{ \pm}\right]\right]=0
$$

Quantization: from $\overline{W_{1+\infty}}$ to $\operatorname{DIM}\left(\mathfrak{g l}_{1}\right)$. This algebra can be deformed with the deformation parameter $\mathfrak{q}$. Let us denote the deformed (properly rescaled) generators through $W_{n}^{0} \rightarrow x_{n}^{0}, W_{n}^{ \pm} \rightarrow x_{n}^{ \pm}$. Then,

$$
\begin{aligned}
& {\left[x_{n}^{0}, x_{m}^{ \pm}\right]=\mp \frac{\kappa_{n}}{n} \mathfrak{q}^{(n \pm|n|) c_{1} / 2} x_{n+m}^{ \pm}} \\
& {\left[x_{n}^{0}, x_{m}^{0}\right]=-\frac{\kappa_{n}}{n} \frac{\mathfrak{q}^{c_{1} n}-\mathfrak{q}^{-c_{1} n}}{\mathfrak{q}-\mathfrak{q}^{-1}} \delta_{n+m, 0}}
\end{aligned}
$$




$$
\left[x_{n}^{+}, x_{m}^{-}\right]=\frac{1}{\kappa_{1}}\left(\mathfrak{q}^{c_{2}+n c_{1}} \psi_{n+m}^{+}-\mathfrak{q}^{-n c_{1}-c_{2}} \psi_{n+m}^{-}\right)
$$

where

$$
\kappa_{n} \equiv\left(q_{1}^{n}-1\right)\left(q_{2}^{n}-1\right)\left(q_{3}^{n}-1\right), \quad q_{1}=\mathfrak{t}^{2}, \quad q_{2}=\mathfrak{q}^{-2} \mathfrak{t}^{-2}, \quad q_{3}=\mathfrak{q}^{2} \quad\left(q_{1} q_{2} q_{3}=1\right)
$$

and

$$
\sum_{k=0}^{\infty} \psi_{k}^{ \pm} z^{\mp k} \equiv \mathfrak{q}^{\mp c_{2}} \exp \left( \pm \sum_{n=1}^{\infty} x_{ \pm n}^{0} z^{\mp n}\right)
$$

Introducing the series of generators,

$$
\psi^{ \pm}(z)=\frac{\left(1-\mathfrak{q}^{2}\right)\left(1-\mathfrak{q}^{-2}\right)}{\kappa_{1}^{2}} \sum_{k=0}^{\infty} \psi_{k}^{ \pm} \mathfrak{q}^{-c_{1} k / 2} z^{\mp k}, \quad x^{ \pm}(z)=\sum_{n \in \mathbb{Z}} x_{n}^{ \pm} z^{-n}
$$

we immediately come to the $\operatorname{DIM}\left(\mathfrak{g l}_{1}\right)$ algebra of section 3.1 upon identification $q_{1}=q$, $q_{2}=t^{-1}$.

Free field realization. At the values of central charges $\left(c_{1}, c_{2}\right)=(1,0)$, the constructed DIM algebra has the deformed affine U(1) subalgebra so that the generators are realized in its terms as

$$
\begin{aligned}
& x^{+}(z)=\exp \left(\sum_{n>0} \frac{1-t^{-n}}{n} \cdot z^{n} p_{n}\right) \cdot \exp \left(\sum_{n>0}\left(q^{n}-1\right) z^{-n} \partial_{p_{n}}\right) \\
& x^{-}(z)=\exp \left(-\sum_{n>0} \frac{1-t^{-n}}{n} \cdot \omega^{-n} z^{n} p_{n}\right) \cdot \exp \left(-\sum_{n>0}\left(q^{n}-1\right) \omega^{-n} z^{-n} \partial_{p_{n}}\right) \\
& \psi^{+}(z)=\exp \left(\sum_{n>0}\left(q^{n}-1\right)\left(1-\omega^{-2 n}\right) z^{-n} \omega^{n / 2} \partial_{p_{n}}\right) \\
& \psi^{-}(z)=\exp \left(\sum_{n>0} \frac{1-t^{-n}}{n}\left(1-\omega^{-2 n}\right) \omega^{n / 2} \cdot z^{n} p_{n}\right)
\end{aligned}
$$

After the Miwa transform of variables $p_{n}=\sum_{i}^{N} z_{i}^{n}$, these expressions reduce to the Macdonald operators

$$
\begin{aligned}
& \left(t^{ \pm 1}-1\right) \sum_{i=1}^{N} \prod_{j(\neq i)} \frac{t^{ \pm 1} z_{i}-z_{j}}{z_{i}-z_{j}} \cdot z_{i}^{n} q^{ \pm \mathcal{D}_{i}} \\
& =\oint \frac{d z}{2 \pi i z} z^{-n}\left\{t^{ \pm N} \exp \left(\sum_{n>0} \frac{1-t^{\mp n}}{n} z^{n} p_{n}\right)-\exp \left(\sum_{n>0} \frac{1-t^{ \pm n}}{n} z^{-n} p_{-n}\right)\right\} \exp \left(\sum_{n>0}\left(q^{ \pm n}-1\right) z^{-n} \partial_{p_{n}}\right)
\end{aligned}
$$

with $\mathcal{D}_{i}:=z_{i} \frac{\partial}{\partial z_{i}}$. Note that the second term in the r.h.s. of the above equation vanishes for $n>0$ so that

$$
\left(t^{ \pm 1}-1\right) \sum_{i=1}^{N} \prod_{j(\neq i)} \frac{t^{ \pm 1} z_{i}-z_{j}}{z_{i}-z_{j}} \cdot z_{i}^{n} q^{ \pm \mathcal{D}_{i}}=(q t)^{\frac{n \mp n}{4}} t^{ \pm N} x_{-n}^{ \pm}-\delta_{n, 0}
$$

with $n \geq 0$. Similarly, at the values of central charges $\left(c_{1}, c_{2}\right)=(2,0)$ this DIM algebra contains a $q$-deformed subalgebra (Virasoro $\otimes \widehat{\mathrm{U}(1)}$ ) (and is realized by two free fields), at $\left(c_{1}, c_{2}\right)=(3,0)$ it contains a $q$-deformed subalgebra $\left(W^{(3)} \otimes \widehat{\mathrm{U}(1)}\right)$ (and is realized by three free fields), etc. 


\section{A.2 Elliptic $\operatorname{DIM}\left(\mathfrak{g l}_{1}\right)$ algebra}

Elliptic version of DIM algebra is generated by the same set of operators as the ordinary DIM: $x^{ \pm}(z), \psi^{ \pm}(z)$ and the central element $\gamma$. The relations are a copy of eq. (3.1), except for the $\left[x^{+}, x^{-}\right]$relation, which changes to

$$
\left[x^{+}(z), x^{-}(w)\right]=\frac{\Theta_{q^{\prime}}\left(q ; q^{\prime}\right) \Theta_{q^{\prime}}\left(t^{-1} ; q^{\prime}\right)}{\left(q^{\prime} ; q^{\prime}\right)_{\infty}^{3} \Theta_{q^{\prime}}\left(q / t ; q^{\prime}\right)}\left(\delta\left(\gamma^{-1} z / w\right) \psi^{+}\left(\gamma^{1 / 2} w\right)-\delta(\gamma z / w) \psi^{-}\left(\gamma^{-1 / 2} w\right)\right)
$$

where $\Theta_{p}(z)=(p ; p)_{\infty}(z ; p)_{\infty}(p / z ; p)_{\infty}$ is the theta-function. Also, most importantly, the structure function $G^{ \pm}(z)$ is now not trigonometric, but elliptic:

$$
G_{\mathrm{ell}}^{ \pm}(z)=\Theta_{p}\left(q^{ \pm 1} z\right) \Theta_{p}\left(t^{\mp 1} z\right) \Theta_{p}\left(q^{\mp 1} t^{ \pm 1} z\right)
$$

The comultiplication $\Delta$ is exactly the same as in the trigonometric case, given by eqs. (3.2). The essential difference with the trigonometric case appears when one tries to build Fock representation of elliptic DIM: one set of bosons turns out not to be enough. One needs at least two sets of Heisenberg generators $\hat{a}_{n}$ and $\hat{b}_{n}$ to reproduce the commutation relations of the elliptic algebra. Concretely, we have for the level one representation:

$$
\begin{aligned}
\rho_{u}\left(x^{+}(z)\right) & =u \eta(z)=u: \exp \left(-\sum_{n \neq 0} \frac{\left(1-t^{n}\right) z^{-n}}{n\left(1-q^{|n|}\right)} \hat{\mathfrak{a}}_{n}\right) \exp \left(-\sum_{n \neq 0} \frac{\left(1-t^{-n}\right) q^{\prime|n|} z^{n}}{n\left(1-q^{|n|}\right)} \hat{\mathfrak{b}}_{n}\right): \\
\rho_{u}\left(x^{-}(z)\right) & =u^{-1} \xi(z)=u^{-1}: \exp \left(\sum_{n \neq 0} \frac{\left(1-t^{n}\right) \omega^{-|n|} z^{-n}}{n\left(1-q^{\prime n \mid}\right)} \hat{\mathfrak{a}}_{n}\right) \exp \left(\sum_{n \neq 0} \frac{\left(1-t^{-n}\right) \omega^{|n|} q^{\prime|n|} z^{n}}{n\left(1-q^{\prime|n|}\right)} \hat{\mathfrak{b}}_{n}\right): \\
\rho_{u}\left(\psi^{+}(z)\right) & =\varphi^{+}(z)=\exp \left(\sum_{n>0} \frac{\left(1-t^{n}\right)\left(\omega^{-n}-\omega^{n}\right) \omega^{-n / 2}}{n\left(1-q^{\prime n}\right)}\left(z^{-n} \hat{\mathfrak{a}}_{n}-\omega^{n} q^{\prime n} z^{n} \hat{\mathfrak{b}}_{n}\right)\right) \\
\rho_{u}\left(\psi^{-}(z)\right) & =\varphi^{-}(z)=\exp \left(-\sum_{n>0} \frac{\left(1-t^{-n}\right)\left(\omega^{-n}-\omega^{n}\right) \omega^{-n / 2}}{n\left(1-q^{\prime n}\right)}\left(z^{n} \hat{\mathfrak{a}}_{-n}-\omega^{n} q^{\prime n} z^{-n} \hat{\mathfrak{b}}_{-n}\right)\right) \\
\rho_{u}(\gamma) & =(t / q)^{1 / 2}
\end{aligned}
$$

where the bosons $\hat{\mathfrak{a}}_{n}$ and $\hat{\mathfrak{b}}_{n}$ satisfy the following commutation relations:

$$
\begin{aligned}
{\left[\hat{\mathfrak{a}}_{m}, \hat{\mathfrak{a}}_{n}\right] } & =m \frac{\left(1-q^{|m|}\right)\left(1-q^{|m|}\right)}{1-t^{|m|}} \delta_{m+n, 0}, \\
{\left[\hat{\mathfrak{b}}_{m}, \hat{\mathfrak{b}}_{n}\right] } & =m \frac{\left(1-q^{\prime|m|}\right)\left(1-q^{|m|}\right)}{\left(p q^{\prime}\right)^{|m|}\left(1-t^{|m|}\right)} \delta_{m+n, 0}, \\
{\left[\hat{\mathfrak{a}}_{m}, \hat{\mathfrak{b}}_{n}\right] } & =0
\end{aligned}
$$

The dressed current $t(z)=\alpha(z) x^{+}(z) \beta(z)$, corresponding to the stress energy tensor is given by exactly the same expression (4.1), as in the ordinary DIM case. Moreover, the dressing operators $\alpha(z)$ and $\beta(z)$ are constructed from the $\psi^{ \pm}$generators of the elliptic DIM algebra using the same formulas (4.2) as give above. In the level two representation $\rho_{u_{1}, u_{2}}^{(2)}$ the element $t(z)$ produces the elliptic Virasoro stress-energy tensor

$$
\mathcal{T}(z)=: e^{\hat{\Phi}(z)} e^{-\hat{\Phi}\left(t^{-1} z\right)}:+t: e^{-\hat{\Phi}(t z / q)} e^{\hat{\Phi}(z / q)}:
$$


where

$$
\hat{\Phi}(z)=\sum_{n \neq 0} \frac{z^{n}}{n\left(1-q^{\prime|n|}\right)} \frac{\hat{\mathfrak{a}}_{-n}}{\sqrt{1+\omega^{|n|}}}-\sum_{n \neq 0} \frac{z^{-n}}{n\left(1-q^{\prime|n|}\right)}\left(\omega^{2} q^{\prime}\right)^{|n| / 2} \hat{\mathfrak{b}}_{-n}
$$

Let us also mention that the undressed elliptic DIM charge $\oint x^{+}(z) d z / z$ also leads to several very interesting objects. In the level one representation it gives elliptic Ruijsenaars Hamiltonian, while in the second level representation it is the difference version of the intermediate long-wave Hamiltonian [197-202], which itself is a generalization of the Benjamin-Ono system.

\section{A.3 Rank > 1: $\operatorname{DIM}\left(\mathfrak{g l}_{n}\right)=$ quantum toroidal algebra of type $\mathfrak{g l}_{n}$}

In complete parallel with the previous consideration, $\operatorname{DIM}\left(\mathfrak{g l}_{n}\right)$ emerge as a deformation of the universal enveloping algebra of the Lie algebra $A_{n}=\operatorname{Mat}_{n} \otimes C\left[z^{ \pm 1}, D^{ \pm 1}\right]$ with

$$
D=q_{1}^{z \frac{\partial}{\partial z}}
$$

i.e. of $n \times n$ matrices with entries being elements of the algebra of functions on the quantum torus, $z D=q_{1} D z$. The deformation of $A_{\mathcal{N}}$ introduces another parameter, $q_{2}$. Providing this deformed algebra with two-dimensional central extension, one arrives at $\operatorname{DIM}\left(\mathfrak{g l}_{n}\right)$.

The set of generators of $\operatorname{DIM}\left(\mathfrak{g l}_{n}\right)$ is $E_{i k}, F_{i k}, H_{i r}, K_{i 0}^{ \pm}, q^{ \pm c}$ with $k \in \mathbb{Z}, r \in \mathbb{Z} /\{0\}$, $0 \leq i \leq n-1$. The generating functions (currents) are:

$$
\begin{aligned}
E_{i}(z) & =\sum_{k \in \mathbb{Z}} E_{i k} z^{-k} \\
F_{i}(z) & =\sum_{k \in \mathbb{Z}} F_{i k} z^{-k} \\
K_{i}^{ \pm}(z) & =K_{i 0}^{ \pm 1} \exp \left( \pm\left(\mathfrak{q}-\mathfrak{q}^{-1}\right) \sum_{r=1}^{\infty} H_{i, \pm r} z^{\mp r}\right)
\end{aligned}
$$

The two centers are $\mathfrak{q}^{c}$ and $\kappa=\prod_{i=0}^{n-1} K_{i 0}$.

The commutation relations are

$$
\begin{aligned}
d_{i j} G_{i j}(z, w) E_{i}(z) E_{j}(w)+G_{j i}(w, z) E_{j}(w) E_{i}(z) & =0, \\
d_{i j} G_{i j}(z, w) K_{i}^{ \pm}\left(\mathfrak{q}^{(1 \mp 1) c / 2} z\right) E_{j}(w)+G_{j i}(w, z) E_{j}(w) K_{i}^{ \pm}\left(\mathfrak{q}^{(1 \mp c) / 2} z\right) & =0, \\
d_{j i} G_{j i}(z, w) F_{i}(z) F_{j}(w)+G_{i j}(w, z) F_{j}(w) F_{i}(z) & =0, \\
d_{j i} G_{j i}(z, w) K_{i}^{ \pm}\left(\mathfrak{q}^{(1 \pm 1) c / 2} z\right) F_{j}(w)+G_{i j}(w, z) F_{j}(w) K_{i}^{ \pm}\left(\mathfrak{q}^{(1 \pm c) / 2} z\right) & =0, \\
{\left[E_{i}(z), F_{j}(w)\right] } & =\frac{\delta_{i j}}{\mathfrak{q}-\mathfrak{q}^{-1}}\left(\delta\left(\frac{\mathfrak{q}^{c} w}{z}\right) K_{i}^{+}(z)-\delta\left(\frac{\mathfrak{q}^{c} z}{w}\right) K_{i}^{-}(w)\right) \\
\frac{G_{i j}\left(\mathfrak{q}^{-c} z, w\right)}{G_{i j}\left(\mathfrak{q}^{c} z, w\right)} K_{i}^{-}(z) K_{j}^{+}(w) & =\frac{G_{j i}\left(w, \mathfrak{q}^{-c} z\right)}{G_{j i}\left(w, \mathfrak{q}^{c} z\right)} K_{i}^{-}(z) K_{j}^{+}(w) \\
{\left[K_{i}^{ \pm}(z), K_{j}^{ \pm}(w)\right] } & =0
\end{aligned}
$$


where, in variance with the $\operatorname{DIM}\left(\mathfrak{g l}_{1}\right)$-case,

$$
q_{1}=\mathfrak{t q}^{-1}, \quad q_{2}=\mathfrak{q}^{2}, \quad q_{3}=\mathfrak{t}^{-1} \mathfrak{q}^{-1}
$$

and powers of $\mathfrak{q}$ are made from entries of the Cartan matrix. The commutation relations can be added with the Serre relations

$$
\begin{array}{rr}
\text { for } n \geq 3 & \operatorname{sym}_{z_{1}, z_{2}}\left[E_{i}\left(z_{1}\right),\left[E_{i}\left(z_{2}\right), E_{i \pm 1}(w)\right]_{\mathfrak{q}}\right]_{\mathfrak{q}^{-1}} \\
\text { for } n=2 & \operatorname{sym}_{z_{1}, z_{2}, z_{3}}\left[E_{i}\left(z_{1}\right),\left[E_{i}\left(z_{2}\right),\left[E_{i}\left(z_{3}\right), E_{i \pm 1}(w)\right]_{\mathfrak{q}^{2}}\right]_{\mathfrak{q}^{0}=1}\right]_{\mathfrak{q}^{-2}}=0
\end{array}
$$

and similarly for $F$. The $q$-commutator is $[A, B]_{\mathfrak{q}}=A B-\mathfrak{q} B A$.

The comultiplication is the same as for $\operatorname{DIM}\left(\mathfrak{g l}_{1}\right)$.

The structure functions are build from the affine Dynkin diagrams and for $\mathfrak{g l}_{n}$-case are defined as follows:

- for the simply laced case $n \geq 3$

$$
\begin{array}{r}
G_{i j}(z, w)=\left\{\begin{array}{c}
\left(z-q_{1} w\right) \text { for } i=j-1 \\
\left(z-q_{2} w\right) \text { for } i=j \\
\left(z-q_{3} w\right) \text { for } i=j+1 \\
(z-w) \text { for } i \neq j, j \pm 1
\end{array}\right. \\
d_{i j}=\left\{\begin{array}{cc}
\mathfrak{t}^{ \pm 1} \text { for } i=j \pm 1, n \geq 3 \\
1 & \text { otherwise }
\end{array}\right.
\end{array}
$$

- The affine Dynkin diagram for $n=2$ is not simply laced, and in this case

$$
\begin{aligned}
G_{00}^{\mathfrak{g l}}(z, w) & =G_{11}^{\mathfrak{g l} l_{2}}(z, w)=\left(z-q_{2} w\right) \\
\widehat{A}_{1} \quad \longleftrightarrow \quad G_{01}^{\mathfrak{g l}_{2}}(z, w) & =G_{10}^{\mathfrak{g l}_{2}}(z, w)=\left(z-q_{1} w\right)\left(z-q_{3} w\right) \\
d_{00} & =d_{11}=1, \quad d_{01}=d_{10}=-1
\end{aligned}
$$

- For $n=1$ we return to section 3.1, i.e.

$$
\bigcirc \quad G_{00}^{\mathfrak{g l}_{1}}(z, w)=\left(z-q_{1} w\right)\left(z-q_{2} w\right)\left(z-q_{3} w\right), \quad d_{00}=1
$$

- One expects in the Pagoda (triple-affine) case $\operatorname{DIM}\left(\widehat{\mathfrak{g l}}_{1}\right)$ (or $U_{q, t, \widehat{t}}\left(\widehat{\widehat{\mathfrak{g l}}}_{1}\right)$, hence, the name Pagoda) the Dynkin diagram of the form:

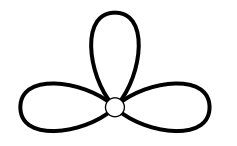

\section{A.4 Affine Yangian of $\mathfrak{g l}_{1}[139]$}

One can consider a "quasiclassical" limit of the $\operatorname{DIM}\left(\mathfrak{g l}_{1}\right)$ algebra, $q=e^{\hbar h_{1}}, t^{-1}=e^{\hbar h_{2}}$, $t / q=e^{\hbar h_{3}}$ with properly rescaled generators. We also use another parameterizations:

$$
\sigma_{1}=h_{1}+h_{2}+h_{3}=0
$$




$$
\begin{aligned}
\sigma_{2} & =h_{1} h_{2}+h_{1} h_{3}+h_{2} h_{3}, \\
\sigma_{3} & =h_{1} h_{2} h_{3}
\end{aligned}
$$

In the limit of $\hbar \rightarrow 0$, one obtains the affine Yangian, which, on the gauge theory side, describes the $4 d$ theories/Nekrasov functions. It is given by the commutation relations:

$$
\begin{aligned}
{\left[e_{i}, f_{j}\right] } & =\psi_{i+j} \\
{\left[\psi_{i}, \psi_{j}\right] } & =0 \\
{\left[e_{i+3}, e_{j}\right]-3\left[e_{i+2}, e_{j+1}\right]+3\left[e_{i+1}, e_{j+2}\right]-\left[e_{i}, e_{j+3}\right] } & \\
+\sigma_{2}\left(\left[e_{i+1}, e_{j}\right]-\left[e_{i}, e_{j+1}\right]\right)-\sigma_{3}\left(e_{i} e_{j}+e_{j} e_{i}\right) & =0 \\
{\left[\psi_{i+3}, e_{j}\right]-3\left[\psi_{i+2}, e_{j+1}\right]+3\left[\psi_{i+1}, e_{j+2}\right]-\left[\psi_{i}, e_{j+3}\right] } & \\
+\sigma_{2}\left(\left[\psi_{i+1}, e_{j}\right]-\left[\psi_{i}, e_{j+1}\right]\right)-\sigma_{3}\left(\psi_{i} e_{j}+\psi_{j} e_{i}\right) & =0
\end{aligned}
$$

and two more relations similar to (A.34) with $e_{i}$ substituted by $f_{i}$ and $\sigma_{3}$ substituted by $-\sigma_{3}$. These commutation relations should be added by the Serre relations

$$
\operatorname{sym}_{i_{1}, i_{2}, i_{3}}\left[e_{i_{1}},\left[e_{i_{2}}, e_{i_{3}+1}\right]\right]=0
$$

and similarly for $f_{i}$.

The commutation relations should be supplemented with the "initial conditions":

- $\psi_{0,1}$ are the central elements, i.e. commute with everything all generators

- $\psi_{2}$ is the grading element, i.e.

$$
\left[\psi_{2}, e_{j}\right]=2 e_{j}, \quad\left[\psi_{2}, f_{j}\right]=-2 f_{j}, \quad\left[\psi_{2}, \psi_{j}\right]=0
$$

Note that, introducing the generator functions

$$
\begin{aligned}
e(u) & =\sum_{i=0}^{\infty} e_{i} u^{-i-1} \\
f(u) & =\sum_{i=0}^{\infty} f_{i} u^{-i-1} \\
\psi(u) & =1+\sigma_{3} \sum_{i=0}^{\infty} \psi_{i} u^{-i-1}
\end{aligned}
$$

one can rewrite the commutation relations as

$$
\begin{aligned}
e(u) e(v) & \sim \Phi(u-v) e(v) e(u), \\
f(u) f(v) & \sim \Phi(v-u) f(v) f(u), \\
\psi(u) e(v) & \sim \Phi(u-v) e(v) \psi(u), \\
\psi(u) f(v) & \sim \Phi(v-u) f(v) \psi(u), \\
e(u) f(v)-f(v) e(u) & \sim-\frac{1}{\sigma_{3}} \frac{\psi(u)-\psi(v)}{u-v}, \\
\psi(u) \psi(v) & \sim \psi(v) \psi(u)
\end{aligned}
$$

with $\Phi(u)=\frac{\left(u+h_{1}\right)\left(u+h_{2}\right)\left(u+h_{3}\right)}{\left(u-h_{1}\right)\left(u-h_{2}\right)\left(u-h_{3}\right)}$. 


\section{Virasoro $\oplus$ Heisenberg subalgebra}

The commutation relations of the Virasoro algebra with extended $\widehat{\mathrm{U}(1)}$-algebra,

$$
\begin{aligned}
{\left[J_{m}, J_{n}\right] } & =k m \delta_{m+n} \\
{\left[L_{m}, J_{n}\right] } & =-n J_{m+n} \\
{\left[L_{m}, L_{n}\right] } & =(m-n) L_{m+n}+\frac{c}{12} n\left(n^{2}-1\right) \delta_{m+n}
\end{aligned}
$$

can be realized with identification:

$$
\begin{aligned}
& J_{-1}=e_{0}, \quad J_{1}=-f_{0}, \\
& L_{-1}=e_{1}+\alpha e_{0}, \quad L_{1}=f_{1}-\alpha f_{0}, \quad \Longrightarrow \quad L_{0}=\psi_{2}+2 \alpha \psi_{1}+\alpha^{2} \psi_{0} \\
& L_{-2}=\frac{1}{2}\left[e_{2}, e_{0}\right]-\frac{1}{2} \beta \sigma_{3} \psi_{0}\left[e_{1}, e_{0}\right], \quad L_{2}=\frac{1}{2}\left[f_{2}, f_{0}\right]+\frac{1}{2} \beta \sigma_{3} \psi_{0}\left[f_{1}, f_{0}\right]
\end{aligned}
$$

From the first line it follows that $k=\psi_{0}$. The other current mode are constructed by repeated commutators: $J_{-2}=\left[e_{1}, e_{0}\right], J_{2}=-\left[f_{1}, f_{0}\right]$ etc. Consistency conditions (e.g. $\left.J_{-3} \sim\left[L_{-1}, J_{-2}\right] \sim\left[L_{-2}, J_{-1}\right]\right)$ require $2 \alpha=(1-\beta) \sigma_{3} \psi_{0}$ (the dependence on $h$-parameters comes from relation with $\left[e_{0}, \psi_{3}\right]$, which does not involve $e_{3}$, because $\left.\left[e_{3}, \psi_{0}\right]=0\right)$. Thus, there remains a free parameter $\beta$.

The central charge is $c=-\sigma_{2} \psi_{0}-\sigma_{3} \psi_{0}^{3}=1-\left(1-\lambda_{1}\right)\left(1-\lambda_{2}\right)\left(1-\lambda_{3}\right)$, where $\lambda_{a}=$ $-\psi_{0} h_{b} h_{c}$ with $(a b c)$ is a cyclic permutation of (123).

\section{Representations: plane partitions}

The basis of a quasi-finite representation of this affine Yangian $^{6}$ can be described by plane partitions ( $3 d$ Young diagrams). The generators of algebra act on the plane partition as follows:

$$
\begin{aligned}
& e(u) \sim \text { adding a box to } 3 d \text { Young diagram } \\
& f(u) \sim \text { removing a box to } 3 d \text { Young diagram } \\
& \psi(u) \sim \text { diagonal action }
\end{aligned}
$$

More precisely,

- the diagonal action is

$$
\begin{aligned}
\psi \mid \Lambda> & =\psi_{\Lambda}(u) \mid \Lambda> \\
\psi_{\Lambda}(u) & =\psi_{\emptyset}(u) \prod_{\square \in \Lambda} \Phi\left(u-u_{0}-h(\square)\right)
\end{aligned}
$$

where $h(\square)=x h_{1}+y h_{2}+z h_{3}$ and $(x, y, z)$ are the coordinates of the box within the plane partition;

\footnotetext{
${ }^{6}$ Such representations are labeled by a triple of ordinary Young diagrams: "minimal" plane partitions are labeled by boundary conditions, [65, 139].
} 
- the raising (lowering) action is

$$
\begin{aligned}
e(u) \mid \Lambda> & =\sum_{\square \in \Lambda_{+} \backslash \Lambda} \frac{E\left(\Lambda \longrightarrow \Lambda_{+}\right)}{u-u_{0}-h(\square)} \mid \Lambda_{+}>, \\
f(u) \mid \Lambda> & =\sum_{\square \in \Lambda \backslash \Lambda_{-}} \frac{F\left(\Lambda \longrightarrow \Lambda_{-}\right)}{u-u_{0}-h(\square)} \mid \Lambda_{-}>
\end{aligned}
$$

where $\Lambda_{+}\left(\Lambda_{-}\right)$denotes arbitrary plane partition with one additional (one subtracted) box as compared to $\Lambda$.

Here $F$ and $E$ are coefficients which have to be defined from the commutation relations of the algebra and are some residues of $\psi_{\Lambda}(u), u_{0}$ is a constant shift, a counterpart of inhomogeneity in the standard spin chain.

Formula (A.42) is derived by acting with the both sides of the commutation relation $\psi(u) e(v) \sim \Phi(u-v) e(v) \psi(u)$ on $\mid \Lambda>$, using (A.43) and then taking the residue at $v=h(\square)$

Constraints on the coefficients $\boldsymbol{E}$ and $\boldsymbol{F}$. Constraints on functions $E\left(\Lambda \longrightarrow \Lambda_{+}\right)$ and $F\left(\Lambda \longrightarrow \Lambda_{-}\right)$can be derived from the commutation relations $\left[e_{i}, f_{j}\right]=\psi_{i+j}$. For the generating functions it looks like

$$
\psi_{\Lambda}(u)=1+\sigma_{3} \sum_{\square} \frac{E\left(\Lambda_{-} \longrightarrow \Lambda\right) F\left(\Lambda \longrightarrow \Lambda_{-}\right)}{u-h(\square)}-\sigma_{3} \sum_{\square} \frac{F\left(\Lambda_{+} \longrightarrow \Lambda\right) E\left(\Lambda \longrightarrow \Lambda_{+}\right)}{u-h(\square)}
$$

where the second-order pole does not contribute. This relation does not fix $E$ and $F$ completely. Imposing an additional requirement of unitarity $E\left(\Lambda \longrightarrow \Lambda_{+}\right)=F\left(\Lambda_{+} \longrightarrow \Lambda\right)$, one immediately obtains [139]

$$
\begin{aligned}
& \sigma_{3} E\left(\Lambda \longrightarrow \Lambda_{+}\right)^{2}=-\operatorname{res}_{u \longrightarrow h(\square)} \psi_{\Lambda}(u) \\
& \sigma_{3} E\left(\Lambda_{-} \longrightarrow \Lambda\right)^{2}=\operatorname{res}_{u \longrightarrow h(\square)} \psi_{\Lambda}(u)
\end{aligned}
$$

One still has to fix the sign (after taking the square root).

The commutation relation $e(u) e(v) \sim \varphi(u-v) e(v) e(u)$ relates adding two boxes in different order:

$$
\frac{E\left(\Lambda \longrightarrow \Lambda+\square_{A}\right) E\left(\Lambda+\square_{A} \longrightarrow \Lambda+\square_{A}+\square_{B}\right)}{E\left(\Lambda \longrightarrow \Lambda+\square_{B}\right) E\left(\Lambda+\square_{B} \longrightarrow \Lambda+\square_{A}+\square_{B}\right)}=\Phi\left(h_{\square_{B}}-h_{\square_{A}}\right)
$$

To check that it is satisfied, calculate the square of the l.h.s.:

$$
\begin{aligned}
& \frac{\operatorname{res}_{u \rightarrow h\left(\square_{A}\right)} \psi_{\Lambda}(u) \cdot \operatorname{res}_{u \rightarrow h\left(\square_{B}\right)} \psi_{\Lambda+\square}(u)}{\operatorname{res}_{u \rightarrow h\left(\square_{B}\right)} \psi_{\Lambda}(u) \cdot \operatorname{res}_{u \rightarrow h\left(\square_{A}\right)} \psi_{\Lambda+\square_{B}}(u)}=\frac{\operatorname{res}_{u \rightarrow h\left(\square_{A}\right)} \psi_{\Lambda}(u) \cdot \operatorname{res}_{u \rightarrow h\left(\square_{B}\right)}\left\{\psi_{\Lambda}(u) \Phi\left(u-h\left(\square_{A}\right)\right)\right\}}{\operatorname{res}_{u \rightarrow h\left(\square_{B}\right)} \psi_{\Lambda}(u) \cdot \operatorname{res}_{u \rightarrow h\left(\square_{A}\right)}\left\{\psi_{\Lambda}(u) \Phi\left(u-h\left(\square_{B}\right)\right)\right\}}= \\
& =\frac{\Phi\left(h\left(\square_{B}\right)-h\left(\square_{A}\right)\right)}{\Phi\left(h\left(\square_{A}\right)-h\left(\square_{B}\right)\right)}=\Phi\left(h\left(\square_{B}\right)-h\left(\square_{A}\right)\right)^{2}
\end{aligned}
$$

Similarly one can check the Serre relations by adding three boxes:

$$
\begin{aligned}
& \sum_{\pi \in S_{3}}\left[h\left(\square_{A_{\pi(1)}}\right)-2 h\left(\square_{A_{\pi(2)}}\right)+h\left(\square_{A_{\pi(3)}}\right)\right] E\left(\Lambda \longrightarrow \Lambda+\square_{A_{\pi(1)}}\right) \times \\
& \times E\left(\Lambda \longrightarrow \Lambda+\square_{A_{\pi(1)}}+\square_{A_{\pi(2)}}\right) E\left(\Lambda \longrightarrow \Lambda+\square_{A_{\pi(1)}}+\square_{A_{\pi(2)}}+\square_{A_{\pi(3)}}\right)=0
\end{aligned}
$$


A simplest example of the highest-weight representation. Consider a representation with the highest weight $\mid \Lambda>$ :

$$
\psi_{j}\left|\Lambda>=\psi_{j, \Lambda}\right| \Lambda>, \quad f_{j} \mid \Lambda>=0
$$

Since we consider the quasi-finite representations, there should be linear relations among $e_{i} \mid \Lambda>$. Consider vectors in the representation at the first level with finitely many, $r-1$ independent vectors. This means that the Shapovalov matrix at the level one, which is

$$
B_{\Lambda}\left(e_{i}, e_{j}\right)<\Lambda|\Lambda>=<\Lambda| f_{i} e_{j}\left|\Lambda>=\psi_{i+j, \Lambda}<\Lambda\right| \Lambda>
$$

should have only $r-1$ independent lines, i.e. there is a relation

$$
\sum_{i=0}^{r-1} \alpha_{i} \psi_{i+k, \lambda}=0, \quad k \geq 0
$$

Then, the generating function of eigenvalues

$$
\psi_{\Lambda}(u)=1+\sigma_{3} \sum_{j=0}^{\infty} \psi_{j, \Lambda} u^{-j-1}=\frac{f(u)}{g(u)}
$$

where $f(u)$ and $g(u)$ are polynomials of degree $r-1$.

Consider the case of $r=2$, i.e. a single state at the level one and linear functions $f(u)$ and $g(u)$ :

$$
\psi_{\lambda}(u)=\frac{u+\sigma_{3} \psi_{0, \lambda}}{u}=1+\frac{\sigma_{3} \psi_{0, \lambda}}{u}
$$

Then, the commutation relations and the Serre relations implies that there are 3 states at the second level (this is since the function $\Phi(u)$ is a ratio of cubic polynomials) and 6 states at the third level. These particular numbers are equal to the number of $3 d$ Young diagrams with a given number of boxes. This means that the highest weight is associated with the trivial plane partition $\mid \lambda>=\emptyset$, and the single first level vector is associated with the only one box plane partition $\mid \square>$ :

$$
e_{i}\left|\emptyset>=0, i>0 ; \quad e_{0}\right| \emptyset>\sim\left|\square>; \quad \psi_{i}\right| \emptyset>=0, i>0
$$

Since $\psi_{1}$ is a center and $\left[\psi_{2}, e_{0}\right]=2 e_{0}$ one immediately obtains

$$
\psi_{1}\left|\square>=0, \quad \psi_{2}\right| \square>=2 \mid \square>
$$

Using these formulas, from the Serre relations that involve $\psi_{j}$ and $e_{0,1,2,3}$, one gets

$$
\psi(u)\left|\square>=\frac{u+\sigma_{3} \psi_{0, \emptyset}}{u} \varphi(u)\right| \square>
$$

Open Access. This article is distributed under the terms of the Creative Commons Attribution License (CC-BY 4.0), which permits any use, distribution and reproduction in any medium, provided the original author(s) and source are credited. 


\section{References}

[1] N. Seiberg and E. Witten, Electric-magnetic duality, monopole condensation and confinement in $N=2$ supersymmetric Yang-Mills theory, Nucl. Phys. B 426 (1994) 19 [Erratum ibid. B 430 (1994) 485] [hep-th/9407087] [INSPIRE].

[2] N. Seiberg and E. Witten, Monopoles, duality and chiral symmetry breaking in $N=2$ supersymmetric QCD, Nucl. Phys. B 431 (1994) 484 [hep-th/9408099] [INSPIRE].

[3] A. Gorsky, I. Krichever, A. Marshakov, A. Mironov and A. Morozov, Integrability and Seiberg-Witten exact solution, Phys. Lett. B 355 (1995) 466 [hep-th/9505035] [INSPIRE].

[4] R. Donagi and E. Witten, Supersymmetric Yang-Mills theory and integrable systems, Nucl. Phys. B 460 (1996) 299 [hep-th/9510101] [InSPIRE].

[5] A. Losev, N. Nekrasov and S.L. Shatashvili, Issues in topological gauge theory, Nucl. Phys. B 534 (1998) 549 [hep-th/9711108] [INSPIRE].

[6] A. Lossev, N. Nekrasov and S.L. Shatashvili, Testing Seiberg-Witten solution, hep-th/9801061 [INSPIRE].

[7] G.W. Moore, N. Nekrasov and S. Shatashvili, Integrating over Higgs branches, Commun. Math. Phys. 209 (2000) 97 [hep-th/9712241] [INSPIRE].

[8] G.W. Moore, N. Nekrasov and S. Shatashvili, D particle bound states and generalized instantons, Commun. Math. Phys. 209 (2000) 77 [hep-th/9803265] [INSPIRE].

[9] N.A. Nekrasov, Seiberg-Witten prepotential from instanton counting, Adv. Theor. Math. Phys. 7 (2003) 831 [hep-th/0206161] [INSPIRE].

[10] R. Flume and R. Poghossian, An algorithm for the microscopic evaluation of the coefficients of the Seiberg-Witten prepotential, Int. J. Mod. Phys. A 18 (2003) 2541 [hep-th/0208176] [INSPIRE].

[11] N. Nekrasov and A. Okounkov, Seiberg-Witten theory and random partitions, Prog. Math. 244 (2006) 525 [hep-th/0306238] [INSPIRE].

[12] A.A. Belavin, A.M. Polyakov and A.B. Zamolodchikov, Infinite conformal symmetry in two-dimensional quantum field theory, Nucl. Phys. B 241 (1984) 333 [InSPIRE].

[13] A. Zamolodchikov and Al. Zamolodchikov, Conformal field theory and critical phenomena in $2 d$ systems, (2009).

[14] L. Álvarez-Gaumé, Random surfaces, statistical mechanics and string theory, Helv. Phys. Acta 64 (1991) 359 [INSPIRE].

[15] P. Di Francesco, P. Mathieu and D. Senechal, Conformal field theory, Springer, Germany (1996).

[16] A. Mironov, S. Mironov, A. Morozov and A. Morozov, CFT exercises for the needs of AGT, Theor. Math. Phys. 165 (2010) 1662 [Teor. Mat. Fiz. 165 (2010) 503] [arXiv:0908. 2064] [INSPIRE].

[17] A. Mironov and A. Morozov, On the origin of Virasoro constraints in matrix models: Lagrangian approach, Phys. Lett. B 252 (1990) 47 [INSPIRE].

[18] H. Itoyama and Y. Matsuo, Noncritical Virasoro algebra of $d<1$ matrix model and quantized string field, Phys. Lett. B 255 (1991) 202 [INSPIRE]. 
[19] R. Dijkgraaf and C. Vafa, Toda theories, matrix models, topological strings and $N=2$ gauge systems, arXiv:0909.2453 [INSPIRE].

[20] H. Itoyama, K. Maruyoshi and T. Oota, The quiver matrix model and 2d-4d conformal connection, Prog. Theor. Phys. 123 (2010) 957 [arXiv:0911.4244] [INSPIRE].

[21] T. Eguchi and K. Maruyoshi, Penner type matrix model and Seiberg-Witten theory, JHEP 02 (2010) 022 [arXiv:0911.4797] [INSPIRE].

[22] T. Eguchi and K. Maruyoshi, Seiberg-Witten theory, matrix model and AGT relation, JHEP 07 (2010) 081 [arXiv: 1006.0828] [INSPIRE].

[23] R. Schiappa and N. Wyllard, An $A_{r}$ threesome: matrix models, $2 d$ CFTs and $4 d N=2$ gauge theories, J. Math. Phys. 51 (2010) 082304 [arXiv:0911.5337] [INSPIRE].

[24] A. Mironov, A. Morozov and S. Shakirov, Matrix model conjecture for exact BS periods and Nekrasov functions, JHEP 02 (2010) 030 [arXiv:0911.5721] [INSPIRE].

[25] A. Mironov, A. Morozov and S. Shakirov, Conformal blocks as Dotsenko-Fateev integral discriminants, Int. J. Mod. Phys. A 25 (2010) 3173 [arXiv:1001.0563] [InSPIRE].

[26] A. Mironov, A. Morozov and S. Shakirov, On 'Dotsenko-Fateev' representation of the toric conformal blocks, J. Phys. A 44 (2011) 085401 [arXiv:1010.1734] [InSPIRE].

[27] A. Mironov, A. Morozov and S. Shakirov, Towards a proof of AGT conjecture by methods of matrix models, Int. J. Mod. Phys. A 27 (2012) 1230001 [arXiv:1011.5629] [InSPIRE].

[28] P. Sulkowski, Matrix models for beta-ensembles from Nekrasov partition functions, JHEP 04 (2010) 063 [arXiv:0912.5476] [INSPIRE].

[29] H. Itoyama and T. Oota, Method of generating q-expansion coefficients for conformal block and $N=2$ Nekrasov function by beta-deformed matrix model, Nucl. Phys. B 838 (2010) 298 [arXiv: 1003.2929] [INSPIRE].

[30] A. Mironov, A. Morozov and A. Morozov, Conformal blocks and generalized Selberg integrals, Nucl. Phys. B 843 (2011) 534 [arXiv:1003.5752] [INSPIRE].

[31] Y. Zenkevich, Generalized Macdonald polynomials, spectral duality for conformal blocks and AGT correspondence in five dimensions, JHEP 05 (2015) 131 [arXiv:1412.8592] [INSPIRE].

[32] A. Morozov and Y. Zenkevich, Decomposing Nekrasov decomposition, JHEP 02 (2016) 098 [arXiv: 1510.01896] [INSPIRE].

[33] A. Mironov, A. Morozov and Y. Zenkevich, On elementary proof of AGT relations from six dimensions, Phys. Lett. B 756 (2016) 208 [arXiv:1512.06701] [INSPIRE].

[34] A. Mironov, A. Morozov and Y. Zenkevich, Spectral duality in elliptic systems, six-dimensional gauge theories and topological strings, JHEP 05 (2016) 121 [arXiv: 1603.00304] [INSPIRE].

[35] A. Iqbal, C. Kozcaz and C. Vafa, The refined topological vertex, JHEP 10 (2009) 069 [hep-th/0701156] [INSPIRE].

[36] H. Awata and H. Kanno, Instanton counting, Macdonald functions and the moduli space of D-branes, JHEP 05 (2005) 039 [hep-th/0502061] [INSPIRE].

[37] H. Awata and H. Kanno, Refined BPS state counting from Nekrasov's formula and Macdonald functions, Int. J. Mod. Phys. A 24 (2009) 2253 [arXiv:0805.0191] [InSPIRE]. 
[38] H. Nakajima, Quiver varieties and t-analogs of q-characters of quantum affine algebras, Ann. Math. 160 (2004) 1057.

[39] H. Nakajima, t-analogs of q-characters of quantum affine algebras of type $A_{n}, D_{n}$, math/0204184.

[40] H. Nakajima, $t$-analogs of q-characters of Kirillov-Reshetikhin modules of quantum affine algebras, math/0204185 [INSPIRE].

[41] H. Awata, B. Feigin, A. Hoshino, M. Kanai, J. Shiraishi and S. Yanagida, Notes on Ding-Iohara algebra and AGT conjecture, arXiv:1106.4088 [INSPIRE].

[42] S. Kanno, Y. Matsuo and S. Shiba, $W_{1+\infty}$ algebra as a symmetry behind AGT relation, Phys. Rev. D 84 (2011) 026007 [arXiv:1105.1667] [InSPIRE].

[43] S. Kanno, Y. Matsuo and H. Zhang, Virasoro constraint for Nekrasov instanton partition function, JHEP 10 (2012) 097 [arXiv: 1207.5658] [INSPIRE].

[44] S. Kanno, Y. Matsuo and H. Zhang, Extended conformal symmetry and recursion formulae for Nekrasov partition function, JHEP 08 (2013) 028 [arXiv:1306.1523] [INSPIRE].

[45] N. Nekrasov and V. Pestun, Seiberg-Witten geometry of four dimensional $N=2$ quiver gauge theories, arXiv:1211.2240 [INSPIRE].

[46] N. Nekrasov, V. Pestun and S. Shatashvili, Quantum geometry and quiver gauge theories, arXiv:1312.6689 [INSPIRE].

[47] N. Nekrasov, BPS/CFT correspondence: non-perturbative Dyson-Schwinger equations and qq-characters, JHEP 03 (2016) 181 [arXiv:1512.05388] [INSPIRE].

[48] T. Kimura and V. Pestun, Quiver $W$-algebras, arXiv:1512.08533 [INSPIRE].

[49] J.-E. Bourgine, Y. Matsuo and H. Zhang, Holomorphic field realization of $S H^{c}$ and quantum geometry of quiver gauge theories, JHEP 04 (2016) 167 [arXiv:1512.02492] [INSPIRE].

[50] A. Mironov, A. Morozov and Y. Zenkevich, Ding-Iohara-Miki symmetry of network matrix models, arXiv: 1603.05467 [INSPIRE].

[51] L.F. Alday, D. Gaiotto and Y. Tachikawa, Liouville correlation functions from four-dimensional gauge theories, Lett. Math. Phys. 91 (2010) 167 [arXiv:0906.3219] [INSPIRE].

[52] N. Wyllard, $A_{N-1}$ conformal Toda field theory correlation functions from conformal $N=2$ $\mathrm{SU}(N)$ quiver gauge theories, JHEP 11 (2009) 002 [arXiv:0907.2189] [INSPIRE].

[53] A. Mironov and A. Morozov, On AGT relation in the case of $\mathrm{U}(3)$, Nucl. Phys. B 825 (2010) 1 [arXiv:0908.2569] [INSPIRE].

[54] J.-T. Ding and K. Iohara, Generalization and deformation of Drinfeld quantum affine algebras, Lett. Math. Phys. 41 (1997) 181 [q-alg/9608002] [INSPIRE].

[55] K. Miki, $A(q, \gamma)$ analog of the $W_{1+\infty}$ algebra, J. Math. Phys. 48 (2007) 123520.

[56] V. Ginzburg, M. Kapranov and E. Vasserot, Langlands reciprocity for algebraic surfaces, Math. Res. Lett. 2 (1995) 147 [q-alg/9502013].

[57] M. Varagnolo and E. Vasserot, Schur duality in the toroidal setting, Commun. Math. Phys. 182 (1996) 469 [q-alg/9506026].

[58] O. Schiffmann and E. Vasserot, The elliptic Hall algebra, Cherednick Hecke algebras and Macdonald polynomials, Compositio Math. 147 (2011) 188 [arXiv:0802.4001]. 
[59] O. Schiffmann and E. Vasserot, The elliptic Hall algebra and the equivariant K-theory of the Hilbert scheme of $A^{2}$, Duke Math. J. 162 (2013) 279 [arXiv:0905.2555].

[60] B. Feigin and A. Tsymbaliuk, Heisenberg action in the equivariant $K$-theory of Hilbert schemes via shuffle algebra, Kyoto J. Math. 51 (2011) 831 [arXiv:0904.1679].

[61] B. Feigin, K. Hashizume, A. Hoshino, J. Shiraishi and S. Yanagida, A commutative algebra on degenerate $C P^{1}$ and Macdonald polynomials, J. Math. Phys. 50 (2009) 095215 [arXiv:0904.2291].

[62] B. Feigin, A. Hoshino, J. Shibahara, J. Shiraishi and S. Yanagida, Kernel function and quantum algebras, arXiv:1002.2485.

[63] B. Feigin, E. Feigin, M. Jimbo, T. Miwa and E. Mukhin, Quantum continuous $\mathfrak{g l}_{\infty}$ : semi-infinite construction of representations, Kyoto J. Math. 51 (2011) 337 [arXiv:1002.3100].

[64] B. Feigin, E. Feigin, M. Jimbo, T. Miwa and E. Mukhin, Quantum continuous $\mathfrak{g l}_{\infty}$ : tensor products of Fock modules and $W_{n}$ characters, Kyoto J. Math. 51 (2011) 365 [arXiv: 1002.3113] [INSPIRE].

[65] B. Feigin, M. Jimbo, T. Miwa and E. Mukhin, Quantum toroidal $\mathfrak{g l}_{1}$ algebra : plane partitions, Kyoto J. Math. 52 (2012) 621 [arXiv:1110.5310].

[66] H. Awata, B. Feigin and J. Shiraishi, Quantum algebraic approach to refined topological vertex, JHEP 03 (2012) 041 [arXiv:1112.6074] [INSPIRE].

[67] B. Feigin, M. Jimbo, T. Miwa and E. Mukhin, Quantum toroidal $\mathfrak{g l}_{1}$ and Bethe ansatz, J. Phys. A 48 (2015) 244001 [arXiv: 1502.07194] [InSPIRE].

[68] B. Feigin, M. Jimbo, T. Miwa and E. Mukhin, Finite type modules and Bethe ansatz for quantum toroidal $\mathfrak{g l}_{1}$, arXiv: 1603.02765.

[69] A. Okounkov and A. Smirnov, Quantum difference equation for Nakajima varieties, arXiv: 1602.09007 [INSPIRE].

[70] A. Marshakov, A. Mironov and A. Morozov, Generalized matrix models as conformal field theories: discrete case, Phys. Lett. B 265 (1991) 99 [InSPIRE].

[71] A. Mironov and S. Pakulyak, On the continuum limit of the conformal matrix models, Theor. Math. Phys. 95 (1993) 604 [Teor. Mat. Fiz. 95 (1993) 317] [Int. J. Mod. Phys. A 8 (1993) 3107] [hep-th/9209100] [INSPIRE].

[72] S. Kharchev, A. Marshakov, A. Mironov, A. Morozov and S. Pakuliak, Conformal matrix models as an alternative to conventional multimatrix models, Nucl. Phys. B 404 (1993) 717 [hep-th/9208044] [INSPIRE].

[73] A. Morozov, String theory: what is it?, Phys. Usp. 35 (1992) 671 [Erratum ibid. 35 (1992) 1003].

[74] A. Morozov, Integrability and matrix models, Phys. Usp. 37 (1994) 1.

[75] A. Morozov, Matrix models as integrable systems, hep-th/9502091 [INSPIRE].

[76] A. Morozov, Challenges of matrix models, hep-th/0502010 [INSPIRE].

[77] A. Mironov, $2 D$ gravity and matrix models. 1. 2D gravity, Int. J. Mod. Phys. A 9 (1994) 4355 [hep-th/9312212] [INSPIRE]. 
[78] A. Mironov, Matrix models of two-dimensional gravity, Phys. Part. Nucl. 33 (2002) 537 [Fiz. Elem. Chast. Atom. Yadra 33 (2002) 1051] [InSPIRE].

[79] A. Mironov, Quantum deformations of tau functions, bilinear identities and representation theory, hep-th/9409190 [INSPIRE].

[80] M. Aganagic, N. Haouzi, C. Kozcaz and S. Shakirov, Gauge/Liouville triality, arXiv: 1309.1687 [INSPIRE].

[81] M. Aganagic, N. Haouzi and S. Shakirov, $A_{n}$-triality, arXiv:1403.3657 [INSPIRE].

[82] M. Aganagic and N. Haouzi, ADE little string theory on a Riemann surface (and triality), arXiv: 1506.04183 [INSPIRE].

[83] B. Feigin, M. Jimbo, T. Miwa and E. Mukhin, Representations of quantum toroidal $\mathfrak{g l}_{n}$, arXiv: 1204.5378 .

[84] B. Feigin, M. Jimbo, T. Miwa and E. Mukhin, Branching rules for quantum toroidal $\mathfrak{g l}_{n}$, arXiv: 1309.2147 [INSPIRE].

[85] A. Tsymbaliuk, Several realizations of Fock modules for quantum toroidal algebras of $s l(n)$, arXiv: 1603.08915.

[86] P. Goddard, A. Kent and D.I. Olive, Virasoro algebras and coset space models, Phys. Lett. B 152 (1985) 88 [INSPIRE].

[87] A. Tsuchiya and Y. Kanie, Vertex operators in the conformal field theory on $P^{1}$ and monodromy representations of the braid group, Lett. Math. Phys. 13 (1987) 303 [INSPIRE].

[88] A. Tsuchiya and Y. Kanie, Vertex operators in conformal field theory on $P^{1}$ and monodromy representations of braid group, Adv. Stud. Pure Math. 16 (1988) 297 [Erratum ibid. 19 (1989) 675] [INSPIRE].

[89] E. Mukhin, V. Tarasov and A. Varchenko, Bispectral and $\left(\mathfrak{g l}_{N}, \mathfrak{g l}_{M}\right)$ dualities, math/0510364.

[90] E. Mukhin, V. Tarasov and A. Varchenko, Bispectral and $\left(\mathfrak{g l}_{N}, \mathfrak{g l}_{M}\right)$ dualities, discrete versus differential, Adv. Math. 218 (2008) 216 [math/0605172].

[91] A. Mironov, A. Morozov, Y. Zenkevich and A. Zotov, Spectral duality in integrable systems from AGT conjecture, JETP Lett. 97 (2013) 45 [arXiv:1204.0913] [Pisma Zh. Eksp. Teor. Fiz. 97 (2013) 49] [INSPIRE].

[92] A. Mironov, A. Morozov, B. Runov, Y. Zenkevich and A. Zotov, Spectral duality between Heisenberg chain and Gaudin model, Lett. Math. Phys. 103 (2013) 299 [arXiv:1206.6349] [INSPIRE].

[93] A. Mironov, A. Morozov, B. Runov, Y. Zenkevich and A. Zotov, Spectral dualities in XXZ spin chains and five dimensional gauge theories, JHEP 12 (2013) 034 [arXiv:1307.1502] [INSPIRE].

[94] L. Bao, E. Pomoni, M. Taki and F. Yagi, M5-branes, toric diagrams and gauge theory duality, JHEP 04 (2012) 105 [arXiv:1112.5228] [INSPIRE].

[95] M. Wakimoto, Fock representations of the affine lie algebra $A_{1}^{(1)}$, Commun. Math. Phys. 104 (1986) 605 [INSPIRE].

[96] A. Gerasimov, A. Morozov, M. Olshanetsky, A. Marshakov and S.L. Shatashvili, Wess-Zumino-Witten model as a theory of free fields, Int. J. Mod. Phys. A 5 (1990) 2495 [INSPIRE]. 
[97] B. Feigin and E. Frenkel, Quantization of the Drinfeld-Sokolov reduction, Phys. Lett. B 246 (1990) 75 [INSPIRE].

[98] A. Tsuchiya and Y. Kanie, Fock space representations of the Virasoro algebra intertwining operators, Publ. R.I.M.S. Kyoto Univ. 22 (1986) 259.

[99] M. Kato and S. Matsuda, Construction of singular vertex operators as degenerate primary conformal fields, Phys. Lett. B 172 (1986) 216 [INSPIRE].

[100] G. Felder, BRST approach to minimal models, Nucl. Phys. B 317 (1989) 215 [Erratum ibid. B 324 (1989) 548] [INSPIRE].

[101] K. Mimachi and Y. Yamada, Singular vectors of the Virasoro algebra in terms of Jack symmetric polynomials, Commun. Math. Phys. 174 (1995) 447.

[102] H. Awata, Y. Matsuo, S. Odake and J. Shiraishi, Collective field theory, Calogero-Sutherland model and generalized matrix models, Phys. Lett. B 347 (1995) 49 [hep-th/9411053] [INSPIRE].

[103] H. Awata, Y. Matsuo, S. Odake and J. Shiraishi, Excited states of Calogero-Sutherland model and singular vectors of the $W_{N}$ algebra, Nucl. Phys. B 449 (1995) 347 [hep-th/9503043] [INSPIRE].

[104] H. Awata, Y. Matsuo, S. Odake and J. Shiraishi, A note on Calogero-Sutherland model, $W_{N}$ singular vectors and generalized matrix models, Soryushiron Kenkyu 91 (1995) A69 [hep-th/9503028] [INSPIRE].

[105] S.H. Katz, A. Klemm and C. Vafa, Geometric engineering of quantum field theories, Nucl. Phys. B 497 (1997) 173 [hep-th/9609239] [InSPIRE].

[106] S. Katz, P. Mayr and C. Vafa, Mirror symmetry and exact solution of $4 D N=2$ gauge theories: 1, Adv. Theor. Math. Phys. 1 (1998) 53 [hep-th/9706110] [INSPIRE].

[107] B. Kol, 5D field theories and M-theory, JHEP 11 (1999) 026 [hep-th/9705031] [INSPIRE].

[108] O. Aharony, A. Hanany and B. Kol, Webs of $(p, q)$ five-branes, five-dimensional field theories and grid diagrams, JHEP 01 (1998) 002 [hep-th/9710116] [INSPIRE].

[109] A. Gorsky, S. Gukov and A. Mironov, SUSY field theories, integrable systems and their stringy/brane origin. 2, Nucl. Phys. B 518 (1998) 689 [hep-th/9710239] [INSPIRE].

[110] B. Kol and J. Rahmfeld, BPS spectrum of five-dimensional field theories, $(p, q)$ webs and curve counting, JHEP 08 (1998) 006 [hep-th/9801067] [INSPIRE].

[111] A. Kapustin, B. Willett and I. Yaakov, Exact results for Wilson loops in superconformal Chern-Simons theories with matter, JHEP 03 (2010) 089 [arXiv:0909.4559] [INSPIRE].

[112] D.L. Jafferis, The exact superconformal R-symmetry extremizes Z, JHEP 05 (2012) 159 [arXiv: 1012.3210] [INSPIRE].

[113] N. Hama, K. Hosomichi and S. Lee, Notes on SUSY gauge theories on three-sphere, JHEP 03 (2011) 127 [arXiv: 1012.3512] [INSPIRE].

[114] C.P. Herzog, I.R. Klebanov, S.S. Pufu and T. Tesileanu, Multi-matrix models and tri-Sasaki Einstein spaces, Phys. Rev. D 83 (2011) 046001 [arXiv:1011.5487] [INSPIRE].

[115] M. Mariño and P. Putrov, ABJM theory as a Fermi gas, J. Stat. Mech. 03 (2012) P03001 [arXiv:1110.4066] [INSPIRE]. 
[116] H. Awata, S. Hirano and M. Shigemori, The partition function of ABJ theory, Prog. Theor. Exp. Phys. 2013 (2013) 053B04 [arXiv:1212.2966] [INSPIRE].

[117] R. Lawrence and L. Rozansky, Witten-Reshetikhin-Turaev invariants of Seifert manifolds, Commun. Math. Phys. 205 (1999) 287.

[118] M. Mariño, Chern-Simons theory, matrix integrals and perturbative three manifold invariants, Commun. Math. Phys. 253 (2004) 25 [hep-th/0207096] [inSPIRE].

[119] M. Aganagic, A. Klemm, M. Mariño and C. Vafa, Matrix model as a mirror of Chern-Simons theory, JHEP 02 (2004) 010 [hep-th/0211098] [INSPIRE].

[120] C. Beasley and E. Witten, Non-Abelian localization for Chern-Simons theory, J. Diff. Geom. 70 (2005) 183 [hep-th/0503126] [INSPIRE].

[121] C. Beasley, Localization for Wilson loops in Chern-Simons theory, Adv. Theor. Math. Phys. 17 (2013) 1 [arXiv: 0911.2687] [INSPIRE].

[122] M. Tierz, Soft matrix models and Chern-Simons partition functions, Mod. Phys. Lett. A 19 (2004) 1365 [hep-th/0212128] [INSPIRE].

[123] A. Brini, B. Eynard and M. Mariño, Torus knots and mirror symmetry, Annales Henri Poincaré 13 (2012) 1873 [arXiv: 1105.2012] [INSPIRE].

[124] A. Alexandrov, A. Mironov, A. Morozov and a. Morozov, Towards matrix model representation of HOMFLY polynomials, JETP Lett. 100 (2014) 271 [arXiv:1407.3754] [INSPIRE].

[125] A. Mironov, A. Morozov and A. Sleptsov, Genus expansion of HOMFLY polynomials, Theor. Math. Phys. 177 (2013) 1435 [Teor. Mat. Fiz. 177 (2013) 179] [arXiv:1303.1015] [INSPIRE].

[126] A. Mironov, A. Morozov and A. Sleptsov, On genus expansion of knot polynomials and hidden structure of Hurwitz tau-functions, Eur. Phys. J. C 73 (2013) 2492 [arXiv: 1304.7499] [INSPIRE].

[127] O. Dubinkin, On the Virasoro constraints for torus knots, J. Phys. A 47 (2014) 485203 [arXiv: 1307.7909] [INSPIRE].

[128] G.W. Moore and N. Seiberg, Classical and quantum conformal field theory, Commun. Math. Phys. 123 (1989) 177 [INSPIRE].

[129] N. Guay, Affine Yangians and deformed double current algebras in type A, Adv. Math. 211 (2007) 436.

[130] D. Maulik and A. Okounkov, Quantum groups and quantum cohomology, arXiv:1211.1287 [INSPIRE].

[131] N. Arbesfeld and O. Schiffmann, A presentation of the deformed $W_{1+\infty}$ algebra, arXiv: 1209.0429 .

[132] O. Schiffmann and E. Vasserot, Cherednik algebras, $W$ algebras and the equivariant cohomology of the moduli space of instantons on $A^{2}$, Publ. Math. Inst. Hautes Etudes Sci. 118 (2013) 213 [arXiv:1202.2756].

[133] A. Smirnov, On the instanton R-matrix, arXiv:1302.0799 [INSPIRE].

[134] A. Smirnov, Polynomials associated with fixed points on the instanton moduli space, arXiv:1404.5304 [INSPIRE]. 
[135] A. Tsymbaliuk, The affine Yangian of $\mathfrak{g l}_{1}$ revisited, arXiv:1404.5240.

[136] R.-D. Zhu and Y. Matsuo, Yangian associated with $2 D N=1$ SCFT, Prog. Theor. Exp. Phys. 2015 (2015) 093A01 [arXiv: 1504.04150] [INSPIRE].

[137] M. Fukuda, S. Nakamura, Y. Matsuo and R.-D. Zhu, SHc realization of minimal model CFT: triality, poset and Burge condition, JHEP 11 (2015) 168 [arXiv:1509.01000] [INSPIRE].

[138] M. Bernshtein and A. Tsymbaliuk, Homomorphisms between different quantum toroidal and affine Yangian algebras, arXiv:1512.09109.

[139] T. Procházka, $W$-symmetry, topological vertex and affine Yangian, arXiv:1512.07178 [INSPIRE].

[140] Y. Saito, Elliptic Ding-Iohara algebra and the free field realization of the elliptic Macdonald operator, arXiv:1301.4912.

[141] Y. Saito, Elliptic Ding-Iohara algebra and commutative families of the elliptic Macdonald operator, arXiv:1309.7094.

[142] A. Iqbal, C. Kozcaz and S.-T. Yau, Elliptic Virasoro conformal blocks, arXiv:1511.00458 [INSPIRE].

[143] F. Nieri, An elliptic Virasoro symmetry in 6d, arXiv:1511.00574 [INSPIRE].

[144] A. Iqbal, All genus topological string amplitudes and five-brane webs as Feynman diagrams, hep-th/0207114 [INSPIRE].

[145] M. Aganagic, A. Klemm, M. Mariño and C. Vafa, The topological vertex, Commun. Math. Phys. 254 (2005) 425 [hep-th/0305132] [INSPIRE].

[146] A. Okounkov, N. Reshetikhin and C. Vafa, Quantum Calabi-Yau and classical crystals, Prog. Math. 244 (2006) 597 [hep-th/0309208] [INSPIRE].

[147] A. Iqbal, N. Nekrasov, A. Okounkov and C. Vafa, Quantum foam and topological strings, JHEP 04 (2008) 011 [hep-th/0312022] [INSPIRE].

[148] A. Iqbal and A.-K. Kashani-Poor, The vertex on a strip, Adv. Theor. Math. Phys. 10 (2006) 317 [hep-th/0410174] [INSPIRE].

[149] H. Nakajima and K. Yoshioka, Instanton counting on blowup. I, Invent. Math. 162 (2005) 313 [math/0306198] [INSPIRE].

[150] H. Nakajima and K. Yoshioka, Instanton counting on blowup. II. K-theoretic partition function, math/0505553 [INSPIRE].

[151] H. Nakajima and K. Yoshioka, Lectures on instanton counting, math/0311058 [INSPIRE].

[152] E. Carlsson, N. Nekrasov and A. Okounkov, Five dimensional gauge theories and vertex operators, arXiv:1308.2465 [INSPIRE].

[153] M. Taki, Refined topological vertex and instanton counting, JHEP 03 (2008) 048 [arXiv: 0710.1776] [INSPIRE].

[154] H. Awata, H. Fuji, H. Kanno, M. Manabe and Y. Yamada, Localization with a surface operator, irregular conformal blocks and open topological string, Adv. Theor. Math. Phys. 16 (2012) 725 [arXiv: 1008.0574] [INSPIRE]. 
[155] H.W. Braden, A. Marshakov, A. Mironov and A. Morozov, On double elliptic integrable systems. 1. A duality argument for the case of SU(2), Nucl. Phys. B 573 (2000) 553 [hep-th/9906240] [INSPIRE].

[156] A. Mironov and A. Morozov, Commuting Hamiltonians from Seiberg-Witten theta functions, Phys. Lett. B 475 (2000) 71 [hep-th/9912088] [INSPIRE].

[157] A. Mironov and A. Morozov, Double elliptic systems: problems and perspectives, hep-th/0001168 [INSPIRE].

[158] G. Aminov, A. Mironov, A. Morozov and A. Zotov, Three-particle integrable systems with elliptic dependence on momenta and theta function identities, Phys. Lett. B 726 (2013) 802 [arXiv: 1307.1465] [INSPIRE].

[159] G. Aminov, H.W. Braden, A. Mironov, A. Morozov and A. Zotov, Seiberg-Witten curves and double-elliptic integrable systems, JHEP 01 (2015) 033 [arXiv:1410.0698] [INSPIRE].

[160] N. Seiberg, Five-dimensional SUSY field theories, nontrivial fixed points and string dynamics, Phys. Lett. B 388 (1996) 753 [hep-th/9608111] [INSPIRE].

[161] M.R. Douglas, S.H. Katz and C. Vafa, Small instantons, del Pezzo surfaces and type-I' theory, Nucl. Phys. B 497 (1997) 155 [hep-th/9609071] [INSPIRE].

[162] D.R. Morrison and N. Seiberg, Extremal transitions and five-dimensional supersymmetric field theories, Nucl. Phys. B 483 (1997) 229 [hep-th/9609070] [INSPIRE].

[163] K.A. Intriligator, D.R. Morrison and N. Seiberg, Five-dimensional supersymmetric gauge theories and degenerations of Calabi-Yau spaces, Nucl. Phys. B 497 (1997) 56 [hep-th/9702198] [INSPIRE].

[164] O. Aharony and A. Hanany, Branes, superpotentials and superconformal fixed points, Nucl. Phys. B 504 (1997) 239 [hep-th/9704170] [INSPIRE].

[165] M. Taki, Notes on enhancement of flavor symmetry and 5d superconformal index, arXiv: 1310.7509 [INSPIRE].

[166] M. Taki, Seiberg duality, 5d SCFTs and Nekrasov partition functions, arXiv:1401.7200 [INSPIRE].

[167] V. Mitev, E. Pomoni, M. Taki and F. Yagi, Fiber-base duality and global symmetry enhancement, JHEP 04 (2015) 052 [arXiv: 1411.2450] [INSPIRE].

[168] S.-S. Kim, M. Taki and F. Yagi, Tao probing the end of the world, Prog. Theor. Exp. Phys. 2015 (2015) 083B02 [arXiv: 1504.03672] [INSPIRE].

[169] H. Hayashi, S.-S. Kim, K. Lee, M. Taki and F. Yagi, A new $5 d$ description of $6 d$ D-type minimal conformal matter, JHEP 08 (2015) 097 [arXiv: 1505.04439] [INSPIRE].

[170] P. Di Francesco, M. Gaudin, C. Itzykson and F. Lesage, Laughlin's wave functions, Coulomb gases and expansions of the discriminant, Int. J. Mod. Phys. A 9 (1994) 4257 [hep-th/9401163] [INSPIRE].

[171] A. Zabrodin, Random matrices and Laplacian growth, arXiv:0907.4929.

[172] A. Morozov and S. Shakirov, The matrix model version of AGT conjecture and CIV-DV prepotential, JHEP 08 (2010) 066 [arXiv: 1004.2917] [INSPIRE].

[173] L. Chekhov, Logarithmic potential $\beta$-ensembles and Feynman graphs, arXiv:1009.5940 [INSPIRE]. 
[174] A. Mironov, A. Morozov, A. Popolitov and S. Shakirov, Resolvents and Seiberg-Witten representation for Gaussian beta-ensemble, Theor. Math. Phys. 171 (2012) 505 [Teor. Mat. Fiz. 171 (2012) 96] [arXiv:1103.5470] [INSPIRE].

[175] H. Awata and Y. Yamada, Five-dimensional AGT conjecture and the deformed Virasoro algebra, JHEP 01 (2010) 125 [arXiv:0910.4431] [INSPIRE].

[176] H. Awata and Y. Yamada, Five-dimensional AGT relation and the deformed beta-ensemble, Prog. Theor. Phys. 124 (2010) 227 [arXiv:1004.5122] [INSPIRE].

[177] A. Mironov, A. Morozov, S. Shakirov and A. Smirnov, Proving AGT conjecture as HS duality: extension to five dimensions, Nucl. Phys. B 855 (2012) 128 [arXiv:1105.0948] [INSPIRE].

[178] H. Itoyama, T. Oota and R. Yoshioka, $q$-Virasoro/W algebra at root of unity and parafermions, Nucl. Phys. B 889 (2014) 25 [arXiv:1408.4216] [INSPIRE].

[179] H. Itoyama, T. Oota and R. Yoshioka, q-vertex operator from $5 D$ Nekrasov function, arXiv:1602.01209 [INSPIRE].

[180] A. Nedelin and M. Zabzine, q-Virasoro constraints in matrix models, arXiv:1511.03471 [INSPIRE].

[181] R. Yoshioka, The integral representation of solutions of $K Z$ equation and a modification by $\mathcal{K}$ operator insertion, arXiv:1512.01084 [INSPIRE].

[182] Y. Zenkevich, Quantum spectral curve for $(q, t)$-matrix model, arXiv: 1507.00519 [INSPIRE].

[183] S. Yanagida, Five-dimensional $\mathrm{SU}(2)$ AGT conjecture and recursive formula of deformed Gaiotto state, J. Math. Phys. 51 (2010) 123506 [arXiv: 1005. 0216] [INSPIRE].

[184] F. Nieri, S. Pasquetti, F. Passerini and A. Torrielli, 5D partition functions, q-Virasoro systems and integrable spin-chains, JHEP 12 (2014) 040 [arXiv:1312.1294] [INSPIRE].

[185] Y. Ohkubo, H. Awata and H. Fujino, Crystallization of deformed Virasoro algebra, Ding-Iohara-Miki algebra and 5D AGT correspondence, arXiv:1512.08016 [INSPIRE].

[186] V.A. Alba, V.A. Fateev, A.V. Litvinov and G.M. Tarnopolskiy, On combinatorial expansion of the conformal blocks arising from AGT conjecture, Lett. Math. Phys. 98 (2011) 33 [arXiv: 1012.1312] [INSPIRE].

[187] A. Belavin and V. Belavin, AGT conjecture and integrable structure of conformal field theory for $c=1$, Nucl. Phys. B 850 (2011) 199 [arXiv:1102.0343] [INSPIRE].

[188] Y. Matsuo, C. Rim and H. Zhang, Construction of Gaiotto states with fundamental multiplets through degenerate DAHA, JHEP 09 (2014) 028 [arXiv:1405.3141] [INSPIRE].

[189] E. Carlsson and A. Okounkov, Exts and vertex operators, arXiv:0801.2565.

[190] A. Negut, Exts and the AGT relations, arXiv:1510.05482 [INSPIRE].

[191] A. Mironov, A. Morozov and S. Shakirov, Brezin-Gross-Witten model as 'pure gauge' limit of Selberg integrals, JHEP 03 (2011) 102 [arXiv:1011.3481] [INSPIRE].

[192] A. Morozov and A. Smirnov, Towards the proof of AGT relations with the help of the generalized Jack polynomials, Lett. Math. Phys. 104 (2014) 585 [arXiv:1307.2576] [INSPIRE].

[193] S. Mironov, A. Morozov and Y. Zenkevich, Generalized Jack polynomials and the AGT relations for the $\mathrm{SU}(3)$ group, JETP Lett. 99 (2014) 109 [arXiv:1312.5732] [INSPIRE]. 
[194] Y. Ohkubo, Existence and orthogonality of generalized Jack polynomials and its q-deformation, arXiv:1404.5401 [INSPIRE].

[195] B. Feigin, M. Jimbo, T. Miwa and E. Mukhin, Quantum toroidal $\mathfrak{g l}_{1}$ and Bethe ansatz, J. Phys. A 48 (2015) 244001 [arXiv: 1502.07194] [INSPIRE].

[196] H. Awata, M. Fukuma, Y. Matsuo and S. Odake, Representation theory of the $W_{1+\infty}$ algebra, Prog. Theor. Phys. Suppl. 118 (1995) 343 [hep-th/9408158] [INSPIRE].

[197] A.V. Litvinov, On spectrum of ILW hierarchy in conformal field theory, JHEP 11 (2013) 155 [arXiv: 1307.8094] [INSPIRE].

[198] M.N. Alfimov and A.V. Litvinov, On spectrum of ILW hierarchy in conformal field theory II: coset CFT's, JHEP 02 (2015) 150 [arXiv:1411.3313] [INSPIRE].

[199] G. Bonelli, A. Sciarappa, A. Tanzini and P. Vasko, Six-dimensional supersymmetric gauge theories, quantum cohomology of instanton moduli spaces and $g l(N)$ quantum intermediate long wave hydrodynamics, JHEP 07 (2014) 141 [arXiv:1403.6454] [INSPIRE].

[200] G. Bonelli, A. Sciarappa, A. Tanzini and P. Vasko, Quantum cohomology and quantum hydrodynamics from supersymmetric quiver gauge theories, arXiv:1505.07116 [INSPIRE].

[201] P. Koroteev and A. Sciarappa, Quantum hydrodynamics from large- $N$ supersymmetric gauge theories, arXiv:1510.00972 [INSPIRE].

[202] P. Koroteev and A. Sciarappa, On elliptic algebras and large- $N$ supersymmetric gauge theories, arXiv: 1601.08238 [INSPIRE]. 